Supporting Information for

\title{
Identifying the Imperative Role of Metal-Olefin Interactions in Catalytic C-O Reductive Elimination from Nickel(II)
}

Trevor D. Lohrey, ${ }^{a}$ Alexander Q. Cusumano, ${ }^{a}$ William A. Goddard III, ${ }^{b, *}$ and Brian M. Stoltz ${ }^{a, *}$

${ }^{a}$ Warren and Katharine Schlinger Laboratory of Chemistry and Chemical Engineering, Division of Chemistry and Chemical Engineering, California Institute of Technology, MC 101-20, Pasadena, California 91125, United States

${ }^{b}$ Materials and Process Simulation Center, Beckman Institute, California Institute of Technology, Pasadena, California 91125, United States

stoltz@caltech.edu

wag@caltech.edu

Table of Contents:

Materials and Methods . $\mathrm{S} 2$

List of Abbreviations . $\mathrm{S} 2$

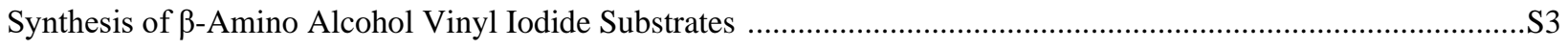

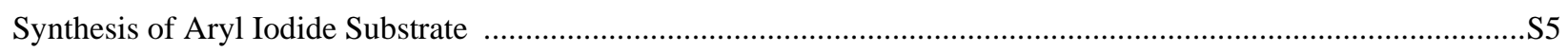

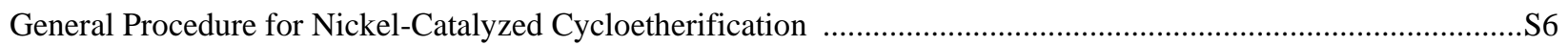

Synthetic Procedures and Characterization Data for Isolated Nickel Compounds ............................................S 8

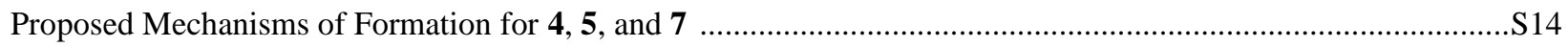

X-Ray Crystallographic Procedures, Refinement Details, and Additional Figures ................................................

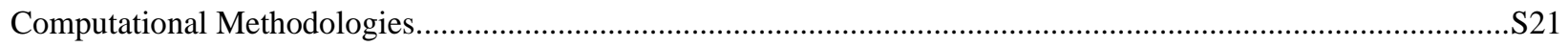

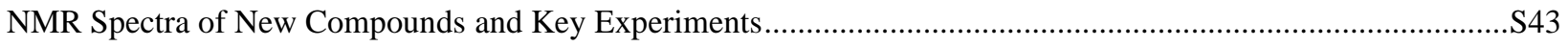

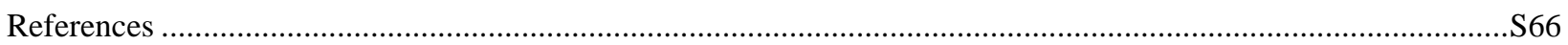




\section{Materials and Methods}

Unless otherwise stated, catalytic and synthetic organometallic reactions were performed in a nitrogen glovebox using dry, deoxygenated solvents. Solvents were dried by passage through an activated alumina column under argon. Organic reagents were purchased from Sigma-Aldrich, Acros Organics, Strem, Oakwood Chemical, or Alfa Aesar and used as received unless otherwise stated. $\mathrm{Ni}(\mathrm{COD})_{2}$ was purchased from Strem and used as received, or recrystallized at $-40{ }^{\circ} \mathrm{C}$ from a $1: 5$ toluene/ $\mathrm{Et}_{2} \mathrm{O}$ mixture prior to use. Reaction progress was monitored by thin-layer chromatography (TLC) or Agilent 1290 UHPLC-MS. TLC was performed using E. Merck silica gel 60 F254 precoated glass plates $(0.25 \mathrm{~mm})$ and visualized by UV fluorescence quenching or $\mathrm{KMnO}_{4}$ staining. Silicycle SiliaFlash® P60 Academic Silica gel (particle size 40-63 $\mu \mathrm{m}$ ) was used for flash column chromatography. ${ }^{1} \mathrm{H}$ NMR spectra were recorded on a Bruker $400 \mathrm{MHz}$ spectrometer and are reported relative to residual $\mathrm{CHCl}_{3}(\delta 7.26$ ppm) or $\mathrm{CHD}_{5}(\delta 7.16 \mathrm{ppm}) .{ }^{13} \mathrm{C}$ NMR spectra were recorded on a Bruker $400 \mathrm{MHz}$ spectrometer $(100 \mathrm{MHz})$ and are reported relative to $\mathrm{CDCl}_{3}(\delta 77.16 \mathrm{ppm})$ or $\mathrm{C}_{6} \mathrm{D}_{6}(\delta 128.06$ ppm). ${ }^{31} \mathrm{P}$ NMR spectra were recorded on a Bruker $400 \mathrm{MHz}$ spectrometer $(162 \mathrm{MHz})$ and are reported relative to a free $\mathrm{PCy}_{3}$ internal standard. Data for ${ }^{1} \mathrm{H}$ NMR are reported as follows: chemical shift $(\delta \mathrm{ppm})$ (multiplicity, coupling constant $(\mathrm{Hz})$, integration). Multiplicities are reported as follows: $\mathrm{s}=$ singlet, $\mathrm{d}=$ doublet, $\mathrm{t}=$ triplet, $\mathrm{q}=$ quartet, $\mathrm{p}=$ pentet, sept $=$ septuplet, $\mathrm{m}=$ multiplet. Data from ${ }^{13} \mathrm{C}$ and ${ }^{31} \mathrm{P}$ NMR spectra are reported in terms of chemical shifts $(\delta$ ppm). IR spectra were obtained by use of a Perkin Elmer Spectrum BXII spectrometer using thin films deposited on $\mathrm{NaCl}$ plates and reported in frequency of absorption $\left(\mathrm{cm}^{-1}\right)$. High resolution mass spectra (HRMS) of new organics were obtained from the Caltech Mass Spectral Facility.

\section{List of Abbreviations:}

TLC - thin-layer chromatography, COD - 1,5-cyclooctadiene, $\mathrm{Et}_{2} \mathrm{O}$ - diethyl ether 


\section{Synthesis of $\beta$-Amino Alcohol Vinyl Iodide Substrates}

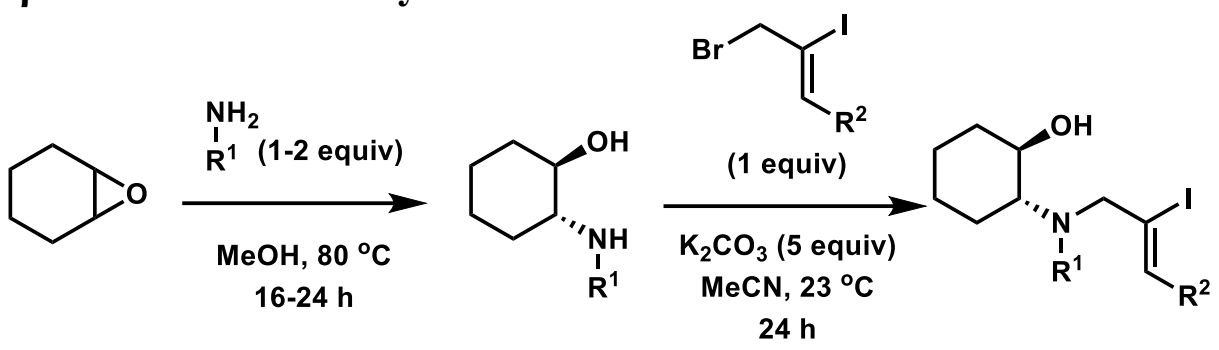

New vinyl iodide substrates $(\mathbf{1 a}, \mathbf{1 d})$ were synthesized by a two-pot procedure akin to that described in our previous report. ${ }^{1}$ A solution of cyclohexene oxide (1.0 equiv) and primary amine (1-2 equiv) in methanol ([amine] $=2 \mathrm{M})$ was heated in a sealed scintillation vial at $80{ }^{\circ} \mathrm{C}$ for 16-24 h, after which time the volatile components of the reaction mixture were removed by rotary evaporation to yield the desired $\beta$-amino alcohols, which were used in the following step without further purification.

To a solution of $\beta$-amino alcohol (1 equiv) dissolved in $\mathrm{MeCN}(5-8 \mathrm{~mL})$ was added $\mathrm{K}_{2} \mathrm{CO}_{3}$ (5 equiv) followed by the appropriate allylic bromide (1 equiv). The resulting mixture was stirred at ambient temperature (ca. $23{ }^{\circ} \mathrm{C}$ ) for $24 \mathrm{~h}$. The reaction mixture was then filtered through Celite and concentrated by rotary evaporation. The resulting crude residues were purified by silica gel chromatography using hexanes/EtOAc as a mobile phase to yield $\beta$-amino alcohol vinyl iodides (1a, 1d).

Compound $\mathbf{3}$ was synthesized by this same procedure, employing cinnamyl bromide in the second step in place of an iodinated allylic bromide.

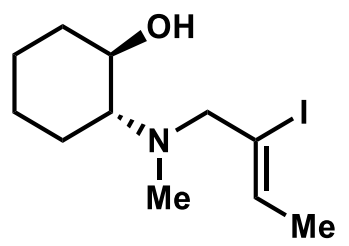

Compound 1a: Isolated as a colorless oil from a $2 \mathrm{mmol}$ scale reaction. Yield: $115 \mathrm{mg}$, 19\%. ${ }^{1} \mathrm{H}$ NMR (400 MHz, $\left.298 \mathrm{~K}, \mathrm{CDCl}_{3}\right): \delta=5.83$ (q, $\left.J=6.0 \mathrm{~Hz}, 1 \mathrm{H}\right), 4.02(\mathrm{~s}, 1 \mathrm{H}), 3.41(\mathrm{td}, J$ $=4.7,9.8 \mathrm{~Hz}, 1 \mathrm{H}), 3.27(\mathrm{~d}, J=13.4 \mathrm{~Hz}, 1 \mathrm{H}), 3.08(\mathrm{~d}, J=13.4 \mathrm{~Hz}, 1 \mathrm{H}), 2.15(\mathrm{~s}, 3 \mathrm{H}), 2.12(\mathrm{td}, J$ $=1.9,5.4 \mathrm{~Hz}, 1 \mathrm{H}), 1.80(\mathrm{~d}, J=\mathrm{Hz}, 3 \mathrm{H}), 1.78-1.62(\mathrm{~m}, 4 \mathrm{H}), 1.33-1.08(\mathrm{~m}, 4 \mathrm{H}) .{ }^{13} \mathrm{C}$ NMR $(100$ $\left.\mathrm{MHz}, 298 \mathrm{~K}, \mathrm{CDCl}_{3}\right): \delta=132.2,111.8,69.5,69.0,65.3,35.7,33.4,25.6,24.3,22.7,21.9$. FT-IR (neat film/NaCl): 3474, 2929, 2854, 1763, 1450, 1304, 1206, 1167, 1081, $1032 \mathrm{~cm}^{-1}$. HRMS (MM: TOF-MS ES+): m/z calc'd for $\mathrm{C}_{11} \mathrm{H}_{21} \mathrm{NOI},[\mathrm{M}+\mathrm{H}]^{+}=310.0668$; found $=310.0662$. 


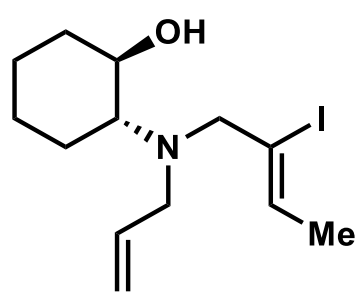

Compound 1d: Isolated as a yellow oil from a $1 \mathrm{mmol}$ scale reaction. Yield: $50 \mathrm{mg}, 15 \%$. ${ }^{1} \mathrm{H}$ NMR (400 MHz, $\left.298 \mathrm{~K}, \mathrm{CDCl}_{3}\right): \delta=5.92-5.78(\mathrm{~m}, 2 \mathrm{H}), 5.14(\mathrm{~d}, J=26.2 \mathrm{~Hz}, 1 \mathrm{H}), 5.13(\mathrm{~s}$, $1 \mathrm{H}), 3.90(\mathrm{~s}, 1 \mathrm{H}), 3.48-3.36(\mathrm{~m}, 2 \mathrm{H}), 3.26(\mathrm{dt}, J=\mathrm{Hz}, 1 \mathrm{H}), 3.02(\mathrm{~d}, J=13.8 \mathrm{~Hz}, 1 \mathrm{H}), 2.91$ (dd, $J$ $=7.4,14.0 \mathrm{~Hz}, 1 \mathrm{H}), 2.44-2.34(\mathrm{~m}, 1 \mathrm{H}), 2.17-2.08(\mathrm{~m}, 1 \mathrm{H}), 1.80(\mathrm{dd}, J=2.6,6.3 \mathrm{~Hz}, 3 \mathrm{H}), 1.78-$ $1.66(\mathrm{~m}, 3 \mathrm{H}), 1.32-1.00(\mathrm{~m}, 4 \mathrm{H}) .{ }^{13} \mathrm{C} \mathrm{NMR}\left(100 \mathrm{MHz}, 298 \mathrm{~K}, \mathrm{CDCl}_{3}\right): \delta=136.8,132.8,117.6$, 111.8, 69.1, 64.7, 60.7, 52.2, 33.3, 25.7, 24.3, 23.4, 22.0. FT-IR (neat film/NaCl): 3485, 2932, 2857, 2358, 1447, 1300, 1206, 1080, 991, 917 cm$^{-1}$. HRMS (MM: TOF-MS ESI+): m/z calc'd for $\mathrm{C}_{13} \mathrm{H}_{23} \mathrm{NOI},[\mathrm{M}+\mathrm{H}]^{+}=336.0824$; found $=336.0813$.

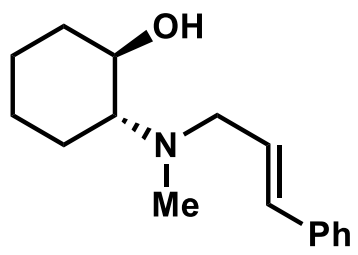

Compound 3: Isolated as a pale yellow semi-crystalline solid from a 1 mmol scale reaction. Yield: $165 \mathrm{mg}, 67 \% .{ }^{1} \mathrm{H}$ NMR (400 MHz, $\left.298 \mathrm{~K}, \mathrm{C}_{6} \mathrm{D}_{6}\right): \delta=7.28-7.23(\mathrm{~m}, 2 \mathrm{H}), 7.16-$ $7.11(\mathrm{~m}, 2 \mathrm{H}), 7.09-7.04(\mathrm{~m}, 1 \mathrm{H}), 6.38(\mathrm{~d}, J=15.9 \mathrm{~Hz}, 1 \mathrm{H}), 6.07$ (ddd, $J=6.3,6.9,15.9 \mathrm{~Hz}, 1 \mathrm{H})$, $3.36(\mathrm{td}, J=4.5,10.4 \mathrm{~Hz}, 1 \mathrm{H}), 3.08(\mathrm{ddd}, J=1.4,6.2,13.7 \mathrm{~Hz}, 1 \mathrm{H}), 2.83(\mathrm{ddd}, J=1.1,7.0,13.7$ $\mathrm{Hz}, 1 \mathrm{H}), 2.29-2.21(\mathrm{~m}, 2 \mathrm{H}), 2.00(\mathrm{~s}, 3 \mathrm{H}), 1.55-1.43$ (m, 3H), 1.42-1.30 (m, 1H), 1.08-0.92 (m, 2H), 0.91-0.79 (m, 1H). ${ }^{13} \mathrm{C}$ NMR (100 MHz, $\left.298 \mathrm{~K}, \mathrm{C}_{6} \mathrm{D}_{6}\right): \delta=137.8,132.6,129.2,129.1$, 127.1, 69.8, 69.8, 56.9, 36.8, 34.4, 26.1, 24.8, 22.2. FT-IR (neat film/NaCl): 3447, 2931, 2857, 1450, 1079, 1030, 965, 743, $693 \mathrm{~cm}^{-1}$. HRMS (MM: TOF-MS ESI+): m/z calc'd for $\mathrm{C}_{16} \mathrm{H}_{24} \mathrm{NO}$, $[\mathrm{M}+\mathrm{H}]^{+}=246.1858 ;$ found $=246.1854$. 


\section{Synthesis of Aryl Iodide Substrate}<smiles>CNc1ccccc1I</smiles>
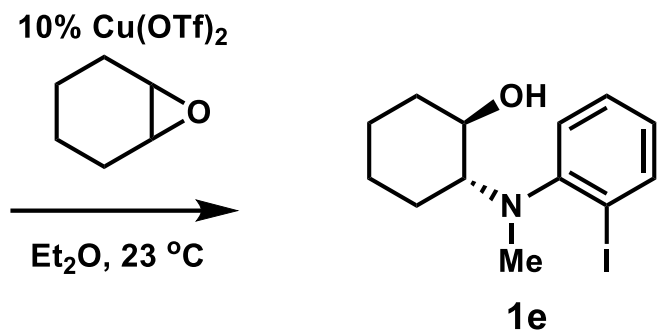

In an adaptation of a reported procedure, ${ }^{2}$ cyclohexene oxide $(197 \mathrm{mg}, 2.01 \mathrm{mmol}, 1.0$

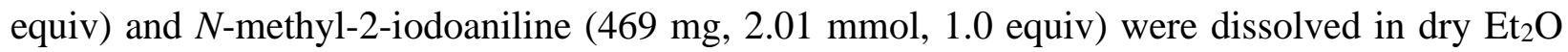
$(10 \mathrm{~mL}) . \mathrm{Cu}(\mathrm{OTf})_{2}(72 \mathrm{mg}, 0.20 \mathrm{mmol}, 0.1$ equiv) was then added, and the reaction mixture was stirred at ca. $23{ }^{\circ} \mathrm{C}$ overnight. The reaction mixture was then filtered through Celite and concentrated by rotary evaporation. Following purification by silica gel chromatography with a hexanes/EtOAc mobile phase, compound 1e was isolated as a colorless oil (189 mg, 29\%).

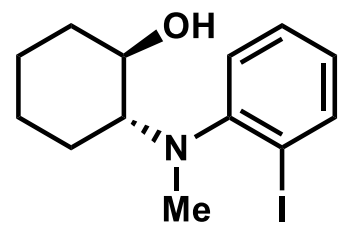

Compound 1e: ${ }^{1} \mathrm{H}$ NMR (400 MHz, $\left.298 \mathrm{~K}, \mathrm{CDCl}_{3}\right): \delta=7.85(\mathrm{dd}, J=1.4,7.9 \mathrm{~Hz}, 1 \mathrm{H})$, 7.34-7.27 (m, 1H), $7.21(\mathrm{dd}, J=1.2,7.9 \mathrm{~Hz}, 1 \mathrm{H}), 6.82(\mathrm{td}, J=1.5,7.3 \mathrm{~Hz}, 1 \mathrm{H}), 3.83(\mathrm{~s}, 1 \mathrm{H})$, $3.65(\mathrm{td}, J=4.2,9.9 \mathrm{~Hz}, 1 \mathrm{H}), 2.89$ (ddd, $J=3.4,9.9,11.7 \mathrm{~Hz}, 1 \mathrm{H}), 2.69$ (s, 3H), 2.21-2.12 (m, $1 \mathrm{H}), 1.87-1.79(\mathrm{~m}, 1 \mathrm{H}), 1.79-1.68(\mathrm{~m}, 2 \mathrm{H}), 1.59-1.46(\mathrm{~m}, 1 \mathrm{H}), 1.40-1.13(\mathrm{~m}, 3 \mathrm{H}) .{ }^{13} \mathrm{C} \mathrm{NMR}(100$ $\left.\mathrm{MHz}, 298 \mathrm{~K}, \mathrm{CDCl}_{3}\right): \delta=153.4,140.3,129.2,126.3,125.2,99.9,70.7,68.2,34.6,33.8,25.5$, 24.5, 24.5. FT-IR (neat film/NaCl): 3486, 2933, 2858, 1578, 1468, 1285, 1076, 1045, 1013, 984, $754 \mathrm{~cm}^{-1}$. HRMS (MM: TOF-MS ESI+): m/z calc'd for $\mathrm{C}_{13} \mathrm{H}_{19} \mathrm{NOI},[\mathrm{M}+\mathrm{H}]^{+}=332.0511$; found $=332.0516$. 


\section{General Procedure for Nickel-Catalyzed Cycloetherification}
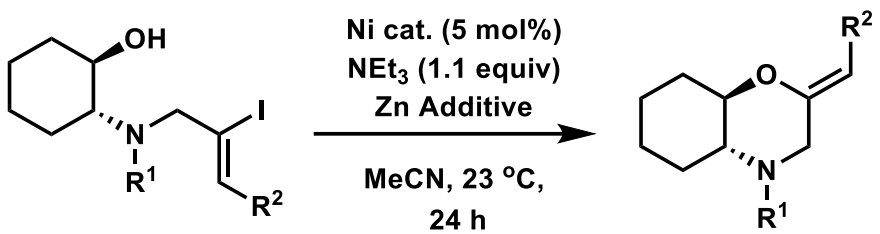

In an $\mathrm{N}_{2}$-filled glovebox, to a vial containing vinyl iodide substrate $(0.135 \mathrm{mmol})$ was added $\mathrm{MeCN}(0.9 \mathrm{~mL}, 0.15 \mathrm{M})$ and triethylamine $(21 \mu \mathrm{L}, 1.1$ equiv). Once a homogeneous solution was obtained by gentle stirring, it was then added by pipette to a 1 dram vial containing Ni precatalyst (e.g. $1.8 \mathrm{mg} / 5 \mathrm{~mol} \%$ of $\mathrm{Ni}(\mathrm{COD})_{2}$ ) and a zinc additive (if applicable, e.g. 17.7 $\mathrm{mg} / 2$ equiv of $\mathrm{Zn}$ dust, or $12.3 \mathrm{mg} / 0.25$ equiv of $\left.\mathrm{Zn}(\mathrm{OTf})_{2}\right)$. The reaction mixture was then stirred in the glovebox at ca. $23{ }^{\circ} \mathrm{C}$ for $24 \mathrm{~h}$ (unless specified otherwise). The reaction mixture was then removed from the glovebox, exposed to ambient atmosphere, filtered through Celite, and concentrated by rotary evaporation. Enol ether products and unreacted starting material were then isolated by silica gel chromatography, using hexanes/EtOAc as a mobile phase. In all cases, the $R_{f}$ values of the enol ether products were found to be significantly lower than those of the vinyl iodide starting materials.

Note that reactions conducted with other Ni precatalysts (e.g. 5, 6, 7) were carried out such that the total $\mathrm{Ni}$ content of the reaction equaled $5 \mathrm{~mol} \%$. Additionally, reactions conducted in solvents other than $\mathrm{MeCN}$ used the same quantity of solvent $(0.9 \mathrm{~mL})$ to maintain an identical starting concentration.

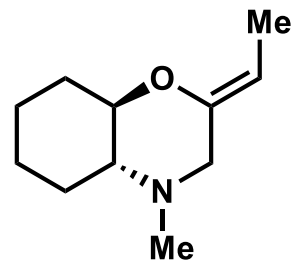

Compound 2a: Isolated as a yellow oil from a $0.135 \mathrm{mmol}$ scale reaction. Reaction time: 24 h. Yield: $13.1 \mathrm{mg}, 54 \%$. ${ }^{1} \mathrm{H}$ NMR (400 MHz, $\left.298 \mathrm{~K}, \mathrm{CDCl}_{3}\right): \delta=4.65(\mathrm{qd}, J=1.5,6.7 \mathrm{~Hz}$, 1H), 3.32 (qd, $J=4.3,9.2 \mathrm{~Hz}, 1 \mathrm{H}), 3.16(\mathrm{~d}, J=12.3 \mathrm{~Hz}, 1 \mathrm{H}), 2.81(\mathrm{~d}, J=11.6 \mathrm{~Hz}, 1 \mathrm{H}), 2.26(\mathrm{~s}$, $3 \mathrm{H}), 2.12-1.99(\mathrm{~m}, 2 \mathrm{H}), 1.91-1.82(\mathrm{~m}, 1 \mathrm{H}) 1.58(\mathrm{dd}, J=2.0,7.7 \mathrm{~Hz}, 3 \mathrm{H}), 1.50-1.38(\mathrm{~m}, 1 \mathrm{H})$, 1.37-1.20 (m, 4H), 1.18-1.05 (m, 1H). $\left.{ }^{13} \mathrm{C} \mathrm{NMR} \mathrm{(100} \mathrm{MHz,} 298 \mathrm{~K}, \mathrm{CDCl}_{3}\right): \delta=148.4,103.8$, 80.6, 67.5, 58.7, 41.5, 31.4, 28.0, 24.8, 24.4, 9.63. FT-IR (neat film/NaCl): 2926, 2861, 1680, 1454, 1361, 1330, 1070, $1026 \mathrm{~cm}^{-1}$. HRMS (MM: TOF-MS ESI+): m/z calc'd for $\mathrm{C}_{11} \mathrm{H}_{20} \mathrm{NO}$, $[\mathrm{M}+\mathrm{H}]^{+}=182.1545 ;$ found $=182.1539$. 


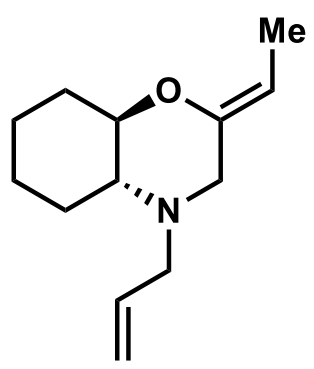

Compound 2d: Isolated as a red-brown oil from a $0.135 \mathrm{mmol}$ scale reaction. Reaction time: $44 \mathrm{~h}$. Yield: $11.4 \mathrm{mg}, 41 \% .50 \%$ of the starting material was also recovered. ${ }^{1} \mathrm{H}$ NMR (400 $\left.\mathrm{MHz}, 298 \mathrm{~K}, \mathrm{CDCl}_{3}\right): \delta=5.86(\mathrm{qdd}, J=5.4,8.1,16.0 \mathrm{~Hz}, 1 \mathrm{H}), 5.21(\mathrm{~m}, 1 \mathrm{H}), 5.17(\mathrm{~s}, 1 \mathrm{H}), 4.61$ $(\mathrm{qd}, J=1.3,6.7 \mathrm{~Hz}, 1 \mathrm{H}), 3.49(\mathrm{dd}, J=5.4,13.6 \mathrm{~Hz}, 1 \mathrm{H}), 3.35(\mathrm{qd}, J=4.3,9.0 \mathrm{~Hz}, 1 \mathrm{H}), 3.24(\mathrm{~d}$, $J=12.5 \mathrm{~Hz}, 1 \mathrm{H}), 2.85-2.75(\mathrm{~m}, 2 \mathrm{H}), 2.17-1.98(\mathrm{~m}, 3 \mathrm{H}), 1.81-1.69(\mathrm{~m}, 2 \mathrm{H}), 1.57$ (dd, $J=1.9,6.7$ $\mathrm{Hz}, 3 \mathrm{H}), 1.50-1.38(\mathrm{~m}, 1 \mathrm{H}), 1.37-1.19(\mathrm{~m}, 3 \mathrm{H}), 1.18-1.07$ (m, 1H). ${ }^{13} \mathrm{C}$ NMR $(100 \mathrm{MHz}, 298 \mathrm{~K}$, $\left.\mathrm{CDCl}_{3}\right): \delta=148.7,134.2,118.9,103.3,80.5,65.3,55.9,54.8,31.6,28.1,24.8,24.4,9.63$. FT-IR (neat film/NaCl): 2937, 2864, 1734, 1678, 1451, 1418, 1330, 1202, 1070, $918 \mathrm{~cm}^{-1}$. HRMS (MM: TOF-MS ESI+): m/z calc'd for $\mathrm{C}_{13} \mathrm{H}_{22} \mathrm{NO},[\mathrm{M}+\mathrm{H}]^{+}=208.1701$; found $=208.1692$.

Table S1. Results of Ni-catalyzed cycloetherification carried out in various solvents.

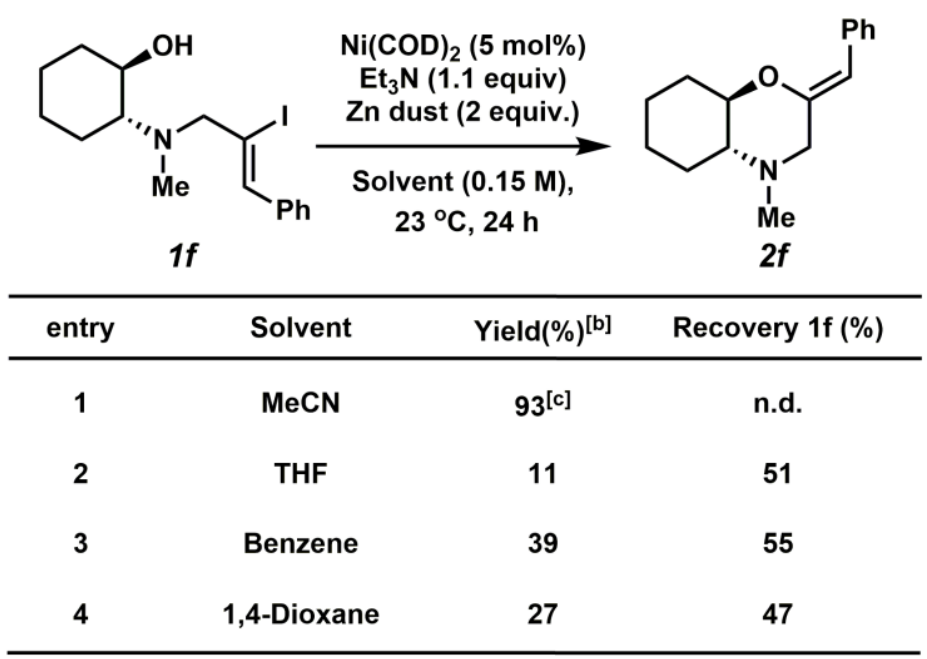

[a] Standard conditions: 1f $(0.135 \mathrm{mmol}), \mathrm{Ni}(\mathrm{COD})_{2}(5 \mathrm{~mol} \%)$, triethylamine $(1.1$ equiv), $\mathrm{Zn}$ dust (2 equiv), Solvent $(0.9 \mathrm{~mL}, 0.15 \mathrm{M})$. [b] Yield of isolated product. [c] Result taken from reference 1 of the Supporting Information. 


\section{Synthetic Procedures and Characterization Data for Isolated Nickel Compounds}

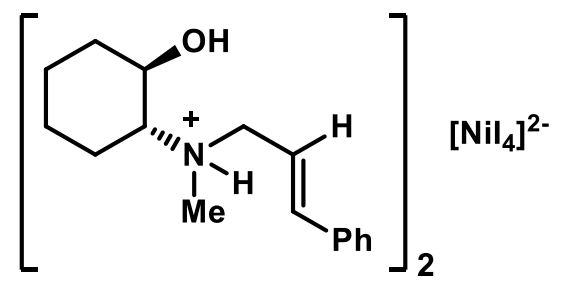

\section{Compound 4}

In an $\mathrm{N}_{2}$-filled glovebox, solid $\mathrm{Ni}(\mathrm{COD})_{2}(37.0 \mathrm{mg}, 0.135 \mathrm{mmol})$ was added to a stirred solution of $\mathbf{1 f}(50 \mathrm{mg}, 0.135 \mathrm{mmol})$ in acetonitrile $(3 \mathrm{~mL})$. Within several minutes, the colorless solution began to take on an orange hue, followed by the precipitation of black solids and the formation of a silvery mirror on the interior of the reaction vial. After a total reaction time of 15 minutes all of the $\mathrm{Ni}(\mathrm{COD})_{2}$ had dissolved, at which point the volatile components of the reaction mixture were removed in vacuo. The crude residue was extracted with benzene $(1 \mathrm{~mL})$. The orange extracts were filtered through Celite into a clean scintillation vial, and the solution was allowed to stand at ambient temperature for several days. Removal of the mother liquor and brief washes with benzene $(2 \times 0.5 \mathrm{~mL})$ yielded 4 as pale orange crystals $(8.7 \mathrm{mg}, 24 \%)$. X-ray quality single crystals of 4 were obtained by a repetition of this procedure. m.p.: $176-179{ }^{\circ} \mathrm{C} .{ }^{1} \mathrm{H}$ NMR (400 MHz, C6 $\left.\mathrm{D}_{6}, 298 \mathrm{~K}\right): \delta 7.79$ (bs), 7.27 (s), 5.54 (bs), 3.57 (bs), 2.91 (bs), 2.24 (bs), 1.84 (bs, overlapped), 1.41 (bs, overlapped), 1.31 (bs, overlapped), 1.23 (bs, overlapped), 0.90 (bm), 0.54 (bs). HRMS (MM: TOF-MS ESI-): $\mathrm{m} / \mathrm{z}$ calc'd for $\mathrm{NiI}_{3}$, $\left[\mathrm{NiI}_{4}-\mathrm{I}\right]^{-}=438.6488$; found = 438.6488 .

Additional anions containing both organic and inorganic fragments were observed in a low resolution ESI- mass spectrum, including $\left[\mathrm{NiI}_{4} \mathrm{C}_{10} \mathrm{H}_{14} \mathrm{~N}\right]^{-}$found at $\mathrm{m} / \mathrm{z}=713.7$ (calc' $\mathrm{d} \mathrm{m} / \mathrm{z}$ $=713.6659$ ) and $\left[\mathrm{NiI}_{3} \mathrm{C}_{10} \mathrm{H}_{13} \mathrm{~N}\right]^{-}$found at $\mathrm{m} / \mathrm{z}=586.0$ (calc'd $\mathrm{m} / \mathrm{z}=585.7536$ ). Both of these anionic species are the result of cyclohexene oxide $\left(\mathrm{C}_{6} \mathrm{H}_{10} \mathrm{O}\right)$ loss from the parent ammonium/ammonia molecule. Given the susceptibility of $\mathbf{4}$ to hydrolysis, HRMS analysis of these anions was unsuccessful. LC-MS analysis of hydrolyzed 4 shows the presence of compound 3 . 


\section{Compound 5}

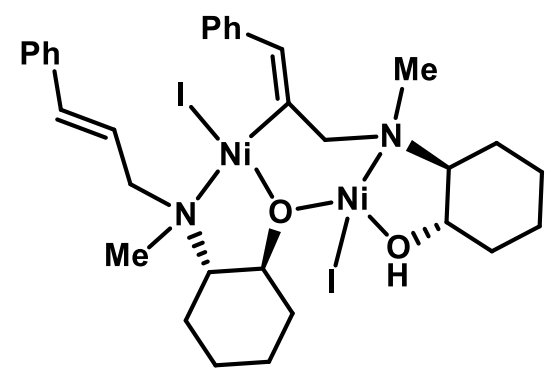

In an $\mathrm{N}_{2}$-filled glovebox, $\mathrm{Ni}(\mathrm{COD})_{2}(74.0 \mathrm{mg}, 0.270 \mathrm{mmol})$ in $\mathrm{Et}_{2} \mathrm{O}(3 \mathrm{~mL})$ was added to a stirred solution of $\mathbf{1 f}(100 \mathrm{mg}, 0.270 \mathrm{mmol})$ in $\mathrm{Et}_{2} \mathrm{O}(5 \mathrm{~mL})$. Within seconds, the solution began to take on an orange hue, and within several minutes, a pale orange precipitate began to form. Fine precipitate continued to form over the course of the reaction. After a reaction time of 45 minutes, the stir bar was removed and the reaction mixture was diluted with hexane, leading to the formation of additional precipitate. After storage of the resulting mixture overnight at $-40{ }^{\circ} \mathrm{C}$, the mother liquor was removed by pipette, and the isolated solids were washed vigorously with hexane $(3 \times 5 \mathrm{~mL})$. Following the removal of residual volatile in vacuo, 5 was isolated as a fine orange powder. X-ray quality single crystals of 5-THF were grown from a THF/hexane solution stored at $-40{ }^{\circ} \mathrm{C}$. Yield: $104 \mathrm{mg}, 82 \%$. m.p.: decomp. without melting above ca. $160{ }^{\circ} \mathrm{C}$. Anal. Calcd. for $\mathrm{C}_{32} \mathrm{H}_{44} \mathrm{~N}_{2} \mathrm{O}_{2} \mathrm{I}_{2} \mathrm{Ni}_{2}$ (5): C, 44.70; H, 5.16; N, 3.26 \%. Found: C, 45.08; H, 5.11; N, 3.19 $\%$. Compound 5 displays a complex, paramagnetically-shifted ${ }^{1} \mathrm{H}$ NMR spectrum that is not amenable to tabulation. 


\section{Compound 6}

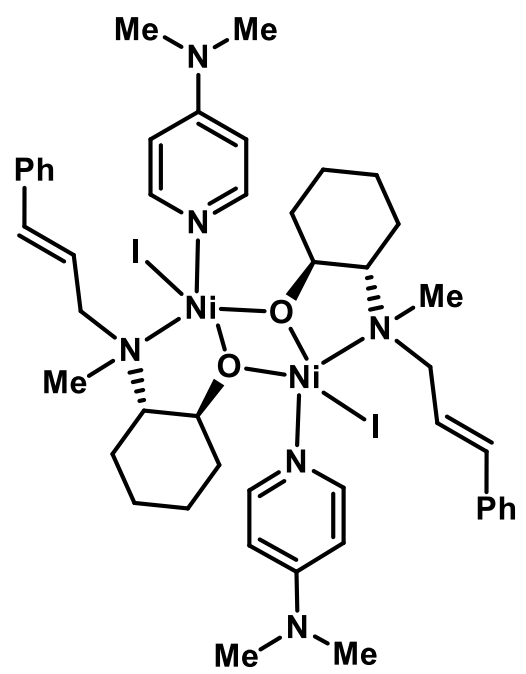

In an $\mathrm{N}_{2}$-filled glovebox, a solid mixture of compound $5(25 \mathrm{mg}, 0.029 \mathrm{mmol})$ and DMAP (7.0 mg, $0.058 \mathrm{mmol})$ was dissolved in benzene $(4 \mathrm{~mL})$. Within seconds, bright orangeyellow crystals began to form. The reaction mixture was allowed to stand at ambient temperature overnight. The following day, the accumulated crystals of 6 were isolated by removing the mother liquor and removing residual volatiles in vacuo. Yield: $27.6 \mathrm{mg}, 86 \%$. X-ray quality single crystals of $\mathbf{6}$ were obtained by repeating the reaction on a smaller scale and lower concentration, slowing the rate of crystallization. m.p. $164-170{ }^{\circ} \mathrm{C}$ (slow melting with decomp.). ${ }^{1} \mathrm{H}$ NMR (400 MHz, $\mathrm{CD}_{2} \mathrm{Cl}_{2}, 298 \mathrm{~K}$ ): $\delta 34.61$ (bs), 29.96 (bs), 24.81 (bs), 20.23 (bs), 18.67 (bs), 8.94 (bs), 7.98 (s), 7.22 (s), 6.79 (s), 6.54 (s), 6.28 (s), 5.76 (s), 5.54 (s), 3.44 (bm), 1.95 (s, overlapped), 1.82 (s, overlapped), 1.27 (s, overlapped), -1.22 (bs), -1.60 (s). Anal. Calcd. for $\mathrm{C}_{64} \mathrm{H}_{82} \mathrm{~N}_{6} \mathrm{O}_{2} \mathrm{I}_{2} \mathrm{Ni}_{2}\left(6 \cdot 3 \mathrm{C}_{6} \mathrm{H}_{6}\right): \mathrm{C}, 57.43 ; \mathrm{H}, 6.17 ; \mathrm{N}, 6.28 \%$. Found: C, 56.63; H, 6.18; N, 6.24 $\%$.

We attribute the low $\mathrm{C}$ wt.\% found in our elemental combustion analysis to the high benzene content of this highly crystalline compound and its incomplete combustion. The presence of three co-crystallized benzene molecules per molecule of $\mathbf{6}$ in the solid state is verified in the X-ray crystal structure. Given this consistency, these three benzene molecules were also accounted for in our calculation of the magnetic moment of this compound. 


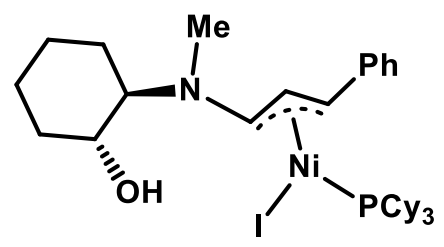

\section{Compound 7}

In an $\mathrm{N}_{2}$-filled glovebox, compound $5(25 \mathrm{mg}, 0.029 \mathrm{mmol})$ was suspended in $\mathrm{Et}_{2} \mathrm{O}$ (4 $\mathrm{mL})$. A solution of tricyclohexylphosphine $(16.3 \mathrm{mg}, 0.058 \mathrm{mmol})$ in $\mathrm{Et}_{2} \mathrm{O}(2 \mathrm{~mL})$ was then added, and the resulting mixture stirred for 20 minutes. The volatile components of the reaction mixture were then removed in vacuo. The crude residue was washed with hexane $(3 \times 2 \mathrm{~mL})$ and exposed to vacuum to remove residual volatiles. Compound 7 was isolated as pale purple-red solids. Yield: $21.3 \mathrm{mg}, 52 \%$. X-ray quality single crystals of 7 were obtained by recrystallizing isolated material from hexane at $-40{ }^{\circ} \mathrm{C}$. m.p.: $148-151{ }^{\circ} \mathrm{C}$. ${ }^{1} \mathrm{H}$ NMR $\left(400 \mathrm{MHz}, \mathrm{C}_{6} \mathrm{D}_{6}, 298 \mathrm{~K}\right): \delta$ $8.35(\mathrm{dq}, J=\mathrm{Hz}, 1 \mathrm{H}), 7.66(\mathrm{~d}, J=\mathrm{Hz}, 2 \mathrm{H}), 7.23(\mathrm{t}, J=\mathrm{Hz}, 2 \mathrm{H}), 7.10(\mathrm{t}, J=\mathrm{Hz}, 1 \mathrm{H}), 6.53(\mathrm{~d}, J=$ Hz, 1H), 4.34 (ddd, $J=\mathrm{Hz}, 1 \mathrm{H}), 2.92$ (td, $J=\mathrm{Hz}, 1 \mathrm{H}), 2.72$ (s, 3H), 2.71-2.64 (m, 1H), 2.37-2.21 (m, 8H), 2.20-2.01 (m, 12H), 1.96-1.84 (m, 5H), 1.84-1.61 (m, 16H), 1.41-1.16 (m, 23H), 1.03$0.94(\mathrm{~m}, 2 \mathrm{H}), 0.91-0.82(\mathrm{~m}, 2 \mathrm{H}), 0.78-0.58(\mathrm{~m}, 2 \mathrm{H}) .{ }^{13} \mathrm{C} \mathrm{NMR}\left(100 \mathrm{MHz}, \mathrm{C}_{6} \mathrm{D}_{6}, 298 \mathrm{~K}\right): \delta$ 137.4, 134.2, 129.0, 127.5, 127.0, 76.3 (d, $J=5.7 \mathrm{~Hz}), 70.2,59.6,39.6,37.3,34.6,34.5(\mathrm{~d}, J=$ $20.8 \mathrm{~Hz}), 30.6(\mathrm{~d}, J=12.7 \mathrm{~Hz}), 29.7,28.2(\mathrm{~d}, J=5.0 \mathrm{~Hz}), 28.1(\mathrm{~d}, J=4.9 \mathrm{~Hz}), 28.1(\mathrm{~d}, J=8.3$ $\mathrm{Hz})$, 27.0, 25.6, 24.8, 22.7. ${ }^{31} \mathrm{P} \mathrm{NMR}\left(162 \mathrm{MHz}, \mathrm{C}_{6} \mathrm{D}_{6}, 298 \mathrm{~K}\right): \delta$ 27.3. Anal. Calcd. for $\mathrm{C}_{34} \mathrm{H}_{55} \mathrm{NOPINi}(7)$ : C, 57.49; H, 7.80; N, $1.97 \%$. Found: C, 57.26; H, 7.83; N, $1.82 \%$.

Compound 7 is isolated as a mixture of diastereomers, in approximately a 1.00:0.28 ratio, which readily co-crystallize (as apparent in the X-ray crystal structure). Based on the NMR spectral data, it appears these diastereomers display different behavior in solution: The major diastereomer of compound 7 displays sharp ${ }^{1} \mathrm{H}$ NMR resonances, while the minor one appears dynamic, leading to broadened NMR peaks. Some ${ }^{1} \mathrm{H}$ NMR resonances, particularly those associated with the $\mathrm{PCy}_{3}$ ligand and cyclohexanol group, are broadened and overlapped: we have listed all of the resonances present in this region of the spectrum, as well as their integrations, which were shown to be reproducible between several batches of material, including after recrystallization or repeated washing of isolated material with hexane. In contrast, the ${ }^{13} \mathrm{C} \mathrm{NMR}$ spectrum of 7 can easily distinguish between the major and minor diastereomers, as the resonances of the major, non-fluxional diastereomer are of significantly greater intensity than those of the fluxional, minor diastereomer. 


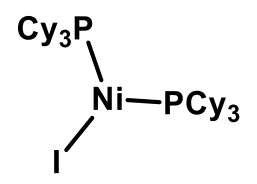

\section{$(\mathbf{P C y})_{2} \mathrm{NiI}$}

While a straightforward synthesis of $\left(\mathrm{PCy}_{3}\right)_{2} \mathrm{NiI}$ is reported in the literature, ${ }^{3}$ the following is an account of our isolation of this material using a vinyl iodide as the halide source. In an $\mathrm{N}_{2}$-filled glovebox, a solid mixture of $\mathrm{Ni}(\mathrm{COD})_{2}(18.5 \mathrm{mg}, 0.067 \mathrm{mmol})$ and tricyclohexylphosphine $(37.8 \mathrm{mg}, 0.135 \mathrm{mmol})$ was dissolved in $\mathrm{Et}_{2} \mathrm{O}(5 \mathrm{~mL})$, forming a clear orange solution. This solution was added to a stirred solution of $\mathbf{1 f}$ ( $25 \mathrm{mg}, 0.067 \mathrm{mmol}$ ) in $\mathrm{Et}_{2} \mathrm{O}$ $(2 \mathrm{~mL}$ ). After ca. $3 \mathrm{~min}$, the reaction mixture began to become cloudy, and over the course of the reaction time (45 min), additional pale precipitate continued to form. The stir bar was then removed from the reaction vial, and the mixture was stored at $-40{ }^{\circ} \mathrm{C}$ overnight. The following day, the mother liquor was removed and the isolated solids were washed with hexane $(3 \mathrm{x} 2 \mathrm{~mL})$. The washed, pale yellow microcrystalline solids $(23.7 \mathrm{mg})$ were then exposed to vacuum to remove residual volatiles. The combined mother liquor and hexane washes were again stored at $40{ }^{\circ} \mathrm{C}$, leading to the formation of a small second crop of product $(4.7 \mathrm{mg})$, which was isolated similarly. Yield: $28.4 \mathrm{mg}, 56 \%$. X-ray quality single crystals of $(\mathrm{PCy})_{2} \mathrm{NiI}$ were obtained by the slow evaporation of a $\mathrm{Et}_{2} \mathrm{O}$ solution of isolated material. ${ }^{1} \mathrm{H} \mathrm{NMR}\left(400 \mathrm{MHz}, \mathrm{C}_{6} \mathrm{D}_{6}, 298 \mathrm{~K}\right): \delta$ 15.50 (bs), 10.04 (bs), 7.54 (bs), 6.74 (bs), 4.15 (bs), 3.30 (bs), 2.97 (bs), 1.93 (bs), 1.67 (bs, overlapped), 1.56 (bs, overlapped), 1.36 (bs, overlapped), 1.08 (bs, overlapped), 0.37 (bs), -1.15 (bs). ${ }^{31} \mathrm{P}$ NMR (162 MHz, $\left.\mathrm{C}_{6} \mathrm{D}_{6}, 298 \mathrm{~K}\right): \delta 46.8$ (bs).

The identity of the isolated product as $\left(\mathrm{PCy}_{3}\right)_{2} \mathrm{NiI}$ was established by $\mathrm{X}$-ray crystallography. Given the lack of reported spectroscopic data for this compound, we herein include its ${ }^{1} \mathrm{H}$ NMR spectrum and ${ }^{31} \mathrm{P}$ NMR spectrum. The X-ray crystal structure of this compound was also previously unknown, and a finalized CIF is also included with this report. 


\section{$[(\mathrm{COD}) \mathrm{NiI}]_{2}$}

The $\mathrm{Ni}(\mathrm{I})$ precatalyst $[(\mathrm{COD}) \mathrm{NiI}]_{2}$ was synthesized by an adaptation of a reported procedure. ${ }^{3}$ In a $\mathrm{N}_{2}$-filled glovebox, $\mathrm{Ni}(\mathrm{COD})_{2}(25.0 \mathrm{mg}, 0.0909 \mathrm{mmol})$ was dissolved in a mixture of toluene $(5 \mathrm{~mL})$ and COD $(0.5 \mathrm{~mL})$. Separately, $\mathrm{I}_{2}(11.5 \mathrm{mg}, 0.0909 \mathrm{mmol})$ was dissolved in $1 \mathrm{~mL}$ toluene. Both solutions were chilled to $-40{ }^{\circ} \mathrm{C}$ in the glovebox freezer. The iodine solution was then added dropwise to the $\mathrm{Ni}(\mathrm{COD})_{2}$ solution with rapid stirring, leading to a change in color of the reaction mixture from light yellow to orange. After stirring for 2 minutes following the addition, the reaction mixture was stored at $-40{ }^{\circ} \mathrm{C}$ overnight to ensure the reaction proceeded to completion without decomposition of the product. The following day, the volatile components of the reaction mixture (now a dark orange solution with notable precipitate) were removed in vacuo, and the resulting residue was washed with diethyl ether $(3 \times 2 \mathrm{~mL})$. $[(\mathrm{COD}) \mathrm{NiI}]_{2}$ was isolated as orange-yellow microcrystalline solids following exposure to vacuum to remove residual volatiles. Yield: $21.8 \mathrm{mg}, 82 \%$. X-ray quality single crystals were grown from a solution of isolated product in a toluene/hexane solution with trace COD at $-40{ }^{\circ} \mathrm{C}$.

While crystalline $[(\mathrm{COD}) \mathrm{NiI}]_{2}$ is somewhat stable at ambient temperature (similarly to $\left.\mathrm{Ni}(\mathrm{COD})_{2}\right)$, we advise that great care must be taken when handling $[(\mathrm{COD}) \mathrm{NiI}]_{2}$ in solution. This highly reactive complex will significantly decompose upon dissolution in benzene or toluene at ambient temperature. The decomposition abates somewhat after an initial generation of dark solids, likely due to the stabilizing effect of free COD in solution. We suggest that any manipulations of this complex are carried out with pre-cooled solvents, with a significant quantity of COD added. We posit the instability of $\left[(\mathrm{COD}) \mathrm{NiI}_{2}\right.$ (particularly when compared to $\left(\mathrm{PCy}_{3}\right)_{2} \mathrm{NiI}$, which is generally stable in solution at room temperature) is due to the lability of $\mathrm{COD}$, as well as the dimeric structure of this complex, which likely predisposes this compound to facile thermal or photoinitiated disproportionation. 
Proposed Mechanisms of Formation for 4, 5, and 7

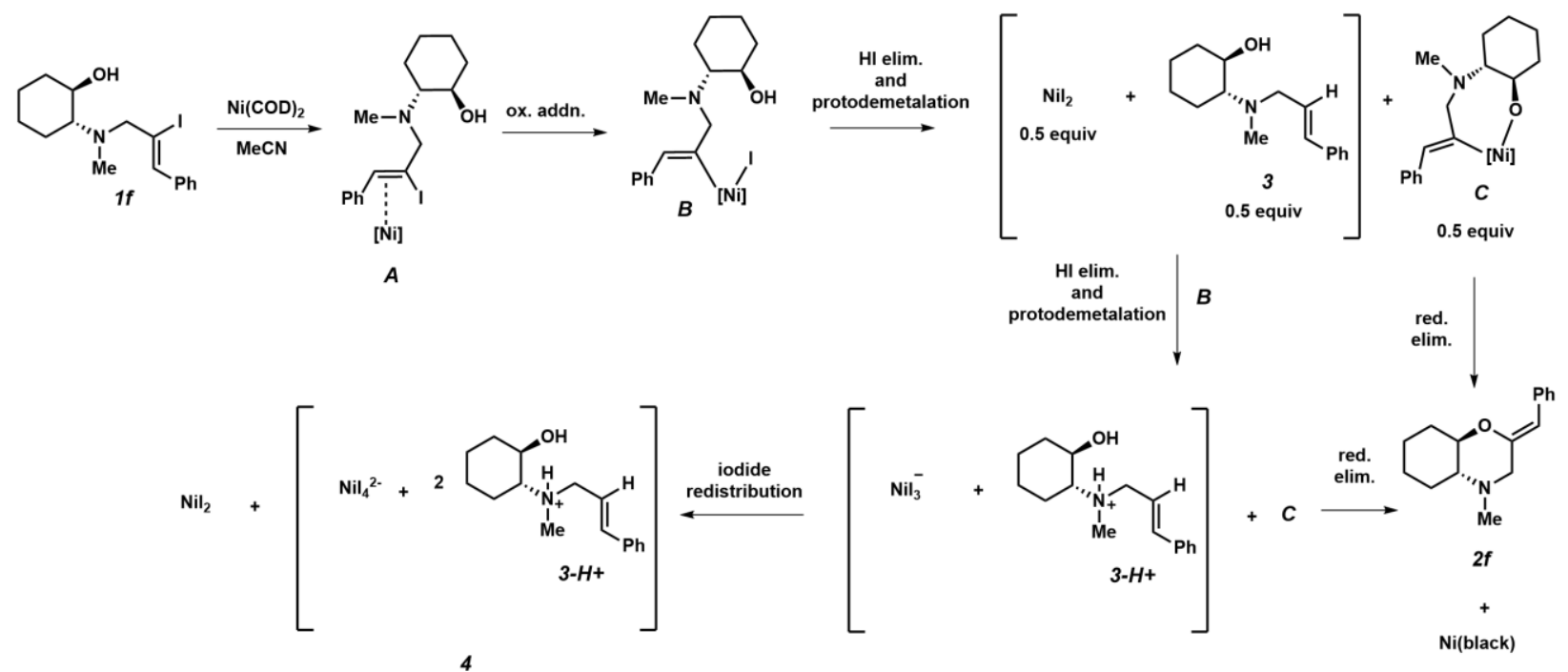

Figure S1. Plausible mechanism for the formation of 3, 4, and $\mathbf{2 f}$ from the stoichiometric reaction of $\mathrm{Ni}(\mathrm{COD})_{2}$ with $\mathbf{1 f}$ in $\mathrm{MeCN}$. [Ni] indicates a solvated $\mathrm{Ni}$ center beyond the ligands explicitly drawn. Note that the generation of $\mathbf{2} \mathbf{f}$ is dependent on the formation of $\mathbf{3}$ via protodemetalation, as the elimination of $\mathrm{HI}$ from intermediate $\mathbf{B}$ (to form $\mathbf{C}$ ) necessitates the presence of a base to deprotonate the free alcohol group. Subsequently, the basic amine group of $\mathbf{3}$, in tandem with $\mathrm{NiI}_{2}$ generated by protodemetalation, may further eliminate $\mathrm{HI}$ from additional equivalents of $\mathbf{B}$, leading to additional quantities of $\mathbf{2} \mathbf{f}$ as well as the isolated ionic compound $\mathbf{4}$.

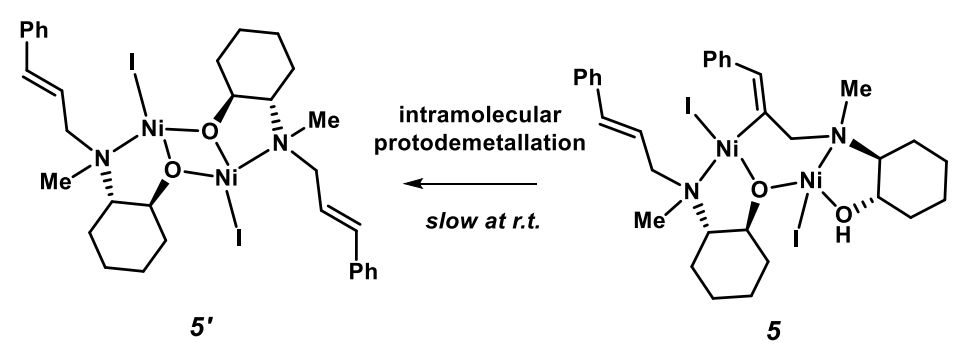

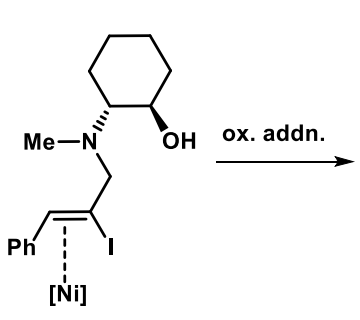

$\boldsymbol{A}$

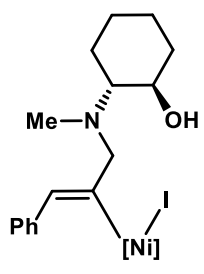

$\boldsymbol{B}$

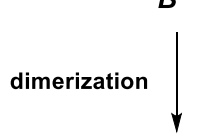

Figure S2. Plausible mechanism for the formation of $\mathbf{5}$ and $\mathbf{5}$ ' from the stoichiometric reaction of $\mathrm{Ni}(\mathrm{COD})_{2}$ with 1 f in $\mathrm{Et}_{2} \mathrm{O}$. [Ni] indicates a solvated $\mathrm{Ni}$ center beyond the ligands explicitly drawn. The key feature of this reaction is the observation that the poorly coordinating nature of $\mathrm{Et}_{2} \mathrm{O}$ leads to the aggregation of $\mathrm{Ni}(\mathrm{II})$ species, by virtue of the favorable chelation of the $\beta$ - 
amino cyclohexanol group (as well as the limited thermodynamic favorability of a single equivalent of $\mathbf{1}$ chelating to a single $\mathrm{Ni}$ center in a tridentate fashion). This dimerization process, which was not observed in $\mathrm{MeCN}$ (likely owing to its much greater coordination strength as compared to that of $\mathrm{Et}_{2} \mathrm{O}$ ), then leads to a rapid internal protodemetalation of one of the $\mathrm{Ni}-\mathrm{C}$ bonds to generate compound $\mathbf{5}$, as isolated. Based on our combined experimental and computational findings, we propose that the slow decomposition of $\mathbf{5}$ at room temperature involves primarily a second protodemetalation, yielding the dimeric alkoxide-bridged $\mathrm{Ni}$ (II) complex $\mathbf{5}$ ' as a major product.

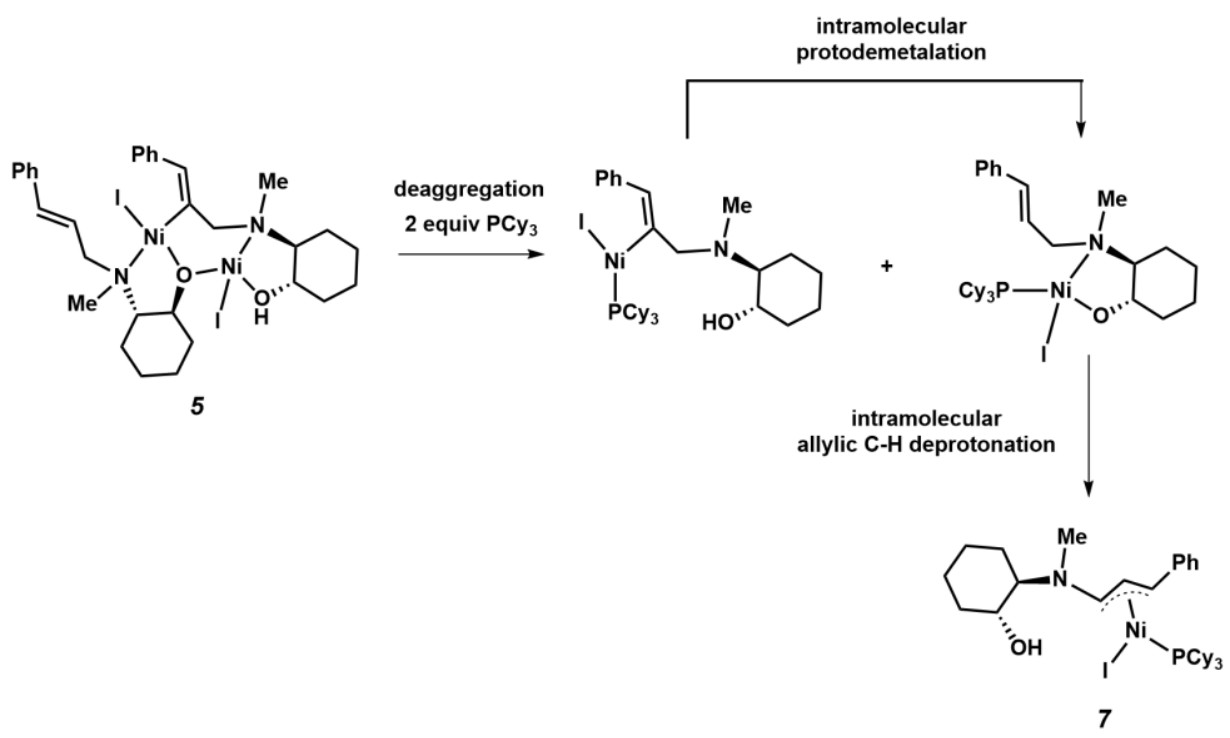

Figure S3. Plausible mechanism for the formation of $\mathbf{7}$ from the reaction of $\mathbf{5}$ with $\mathrm{PCy}_{3}$. Note that the allylic deprotonation likely depends on the formation of mononuclear $\mathrm{Ni}$ (II) intermediates, as the structures of compounds $\mathbf{5}$ and $\mathbf{6}$ indicate that bridging alkoxide groups are not amenable to promoting this process. 


\section{X-Ray Crystallographic Procedures, Refinement Details, and Additional Figures}

Single crystal X-ray diffraction data for 4, 5-THF, 6, 7, $\left[(\mathrm{COD}) \mathrm{NiI}_{2}\right.$, and $(\mathrm{PCy})_{2} \mathrm{NiI}$ were collected at the Caltech XRCF, using either a Bruker AXS D8 Venture outfitted with a rotating anode Mo-K $\alpha(\lambda=0.71073 \AA)$ micro-source, or a Bruker AXS D8 KAPPA with a sealed tube Mo-K $\alpha$ X-ray source. All data collections were conducted at $100 \mathrm{~K}$ in a dry nitrogen cryostream. In an $\mathrm{N}_{2}$-filled glovebox, samples of single crystals were isolated from their mother liquor and coated in a thick hydrocarbon oil for transport to the diffraction facility. In the facility, samples were evaluated by polarized light microscopy, and candidate crystals were mounted on an appropriately-sized MiTiGen MicroLoop for X-ray diffraction analysis. Diffraction data were collected, integrated, and reduced using Bruker AXS APEX3 software, including its associated SAINT and SADABS programs. ${ }^{4}$ Structures were solved and refined using SHELXT and SHELXL-2014, ${ }^{5,6}$ as implemented within WinGX. ${ }^{7}$ The structural model for the allyl complex 7 required modeling of the cyclohexanol moiety over two positions (due to co-crystallization of diastereotopic Ni complexes containing different stereoisomers of the racemic trans- $\beta$-amino alcohol). The structural model for the asymmetric dinuclear Ni complex 5-THF required disorder modeling for multiple co-crystallized THF molecules. The PLATON squeeze program ${ }^{8}$ was used to remove electron density associated with a highly disordered $\mathrm{Et}_{2} \mathrm{O}$ molecule in the structure of $\left(\mathrm{PCy}_{3}\right)_{2} \mathrm{NiI}$ oriented on a 3 -fold screw axis (along $c$ at $a=b=1 / 3$, these void channels are readily observed in a packing diagram of the final model). Thermal ellipsoid plots were generated using Mercury. ${ }^{9}$ Finalized CIFs have been deposited to the Cambridge Crystallographic Data Centre (CCDC), with deposition numbers: 2078291 (4), 2078292 (5THF), 2078293 (6), 2078294 (7), 2078295 ((PCy $\left.)_{2} \mathrm{NiI}\right)$, and $2088878\left(\left[(\mathrm{COD}) \mathrm{NiI}_{2}\right)\right.$. 
Table S2. Crystallographic details and refinement metrics.

\begin{tabular}{|c|c|c|c|c|c|c|}
\hline & $4 \cdot 2\left(\mathrm{C}_{6} \mathrm{H}_{6}\right)$ & $\begin{array}{l}\text { 5-THF・ } \\
\text { 3.5(THF) }\end{array}$ & $6 \cdot 3\left(\mathrm{C}_{6} \mathrm{H}_{6}\right)$ & 7 & {$\left[(\mathrm{COD}) \mathrm{NiI}_{2}\right.$} & $\begin{array}{l}\left(\mathbf{P C y}_{3}\right)_{2} \mathbf{N i I} \\
\mathbf{E t}_{2} \mathbf{O}\end{array}$ \\
\hline Chemical formula & $\begin{array}{l}\mathrm{C}_{44} \mathrm{H}_{60} \mathrm{I}_{4} \mathrm{~N}_{2} \mathrm{Ni} \\
\mathrm{O}_{2}\end{array}$ & $\begin{array}{l}\mathrm{C}_{50} \mathrm{H}_{80} \mathrm{I}_{2} \mathrm{~N}_{2} \mathrm{Ni}_{2} \\
\mathrm{O}_{6.5}\end{array}$ & $\mathrm{C}_{64} \mathrm{H}_{82} \mathrm{I}_{2} \mathrm{~N}_{6} \mathrm{Ni}_{2} \mathrm{O}_{2}$ & $\mathrm{C}_{34} \mathrm{H}_{55} \mathrm{INNiOP}$ & $\mathrm{C}_{16} \mathrm{H}_{24} \mathrm{I}_{2} \mathrm{Ni}_{2}$ & $\mathrm{C}_{36} \mathrm{H}_{66} \mathrm{INiP}_{2}$ \\
\hline Formula weight & 1215.25 & 1184.38 & 1338.57 & 710.37 & 587.57 & 746.43 \\
\hline Color, habit & Orange, block & Orange, lath & Orange, block & Purple, block & Orange, needle & Colorless, rod \\
\hline Temperature (K) & $100(2)$ & $100(2)$ & $100(2)$ & $100(2)$ & $100(2)$ & $100(2)$ \\
\hline Crystal system & Monoclinic & Triclinic & Orthorhombic & Triclinic & Monoclinic & Rhombohedral \\
\hline Space group & $\mathrm{C} 2 / \mathrm{c}$ & P-1 & Pccn & $\mathrm{P}-1$ & $\mathrm{P} 2{ }_{1} / \mathrm{n}$ & $\mathrm{R}-3 \mathrm{c}$ \\
\hline $\mathrm{a}(\AA)$ & $27.330(11)$ & $12.7775(13)$ & $14.7031(9)$ & $11.0239(9)$ & $7.4617(17)$ & $29.259(3)$ \\
\hline $\mathrm{b}(\AA)$ & $9.978(4)$ & $14.7475(15)$ & $17.8228(11)$ & 11.4716(9) & $10.686(2)$ & $29.259(3)$ \\
\hline c $(\AA)$ & $17.222(6)$ & $15.2774(16)$ & $23.8035(15)$ & $13.6693(11)$ & $10.939(3)$ & $23.165(2)$ \\
\hline$\alpha\left(^{\circ}\right)$ & 90 & $73.188(4)$ & 90 & $81.983(3)$ & 90 & 90 \\
\hline$\beta\left(^{\circ}\right)$ & $90.618(13)$ & $86.253(4)$ & 90 & $78.073(3)$ & $97.511(10)$ & 90 \\
\hline$\gamma\left({ }^{\circ}\right)$ & 90 & $70.648(4)$ & 90 & $75.856(3)$ & 90 & 120 \\
\hline$V\left(\AA^{3}\right)$ & $4696(3)$ & $2598.6(5)$ & $6237.7(7)$ & $1632.8(2)$ & $864.8(4)$ & $17175(4)$ \\
\hline $\mathrm{Z}$ & 4 & 2 & 4 & 2 & 2 & 18 \\
\hline Density $\left(\mathrm{Mg} \mathrm{m}^{-3}\right)$ & 1.719 & 1.514 & 1.425 & 1.455 & 2.257 & 1.299 \\
\hline $\mathrm{F}(000)$ & 2376 & 1216 & 2744 & 740 & 564 & 7074 \\
\hline Radiation Type & $\mathrm{MoK}_{\alpha}$ & $\mathrm{MoK}_{\alpha}$ & $\mathrm{MoK}_{\alpha}$ & $\operatorname{MoK}_{\alpha}$ & $\operatorname{MoK}_{\alpha}$ & $\operatorname{MoK}_{\alpha}$ \\
\hline$\mu\left(\mathrm{mm}^{-1}\right)$ & 3.078 & 1.961 & 1.640 & 1.615 & 5.735 & 1.423 \\
\hline $\begin{array}{l}\text { Crystal } \\
\left(\mathrm{mm}^{3}\right)\end{array} \quad$ size & $\begin{array}{l}0.070 \times 0.050 \\
\times 0.040\end{array}$ & $\begin{array}{l}0.200 \times 0.100 \\
\times 0.030\end{array}$ & $\begin{array}{l}0.250 \times 0.230 \times \\
0.180\end{array}$ & $\begin{array}{l}0.120 \times 0.090 \times \\
0.050\end{array}$ & $\begin{array}{l}0.100 \times 0.020 \times \\
0.015\end{array}$ & $\begin{array}{l}0.120 \times 0.040 \\
\times 0.030\end{array}$ \\
\hline Meas. Refl. & 28482 & 110737 & 48347 & 44904 & 13084 & 85598 \\
\hline Indep. Refl. & 4296 & 9509 & 5444 & 6718 & 1774 & 3978 \\
\hline $\mathrm{R}$ (int) & 0.1150 & 0.0307 & 0.0399 & 0.0701 & 0.0769 & 0.0742 \\
\hline $\begin{array}{l}\text { Completeness to } \\
\arcsin (0.6 * \lambda)\end{array}$ & $99.7 \%$ & $99.5 \%$ & $98.6 \%$ & $100.0 \%$ & $100.0 \%$ & $99.9 \%$ \\
\hline Final R indices & $\mathrm{R}=0.0650$ & $\mathrm{R}=0.0483$ & $\mathrm{R}=0.0224$ & $\mathrm{R}=0.0425$ & $\mathrm{R}=0.0278$ & $\mathrm{R}=0.0320$ \\
\hline$[\mathrm{I}>2 \sigma(\mathrm{I})]$ & $\mathrm{R}_{\mathrm{w}}=0.1943$ & $\mathrm{R}_{\mathrm{w}}=0.1310$ & $\mathrm{R}_{\mathrm{w}}=0.0460$ & $\mathrm{R}_{\mathrm{w}}=0.0915$ & $\mathrm{R}_{\mathrm{w}}=0.0499$ & $\mathrm{R}_{\mathrm{w}}=0.0861$ \\
\hline Goodness-of-fit & 1.075 & 1.127 & 1.080 & 1.028 & 1.052 & 1.067 \\
\hline $\begin{array}{l}\Delta \rho_{\max }, \Delta \rho_{\min } \\
\left(\mathrm{e} \AA^{-3}\right)\end{array}$ & $4.941,-1.200$ & $1.433,-0.913$ & $0.416,-0.453$ & $0.879,-0.593$ & $0.647,-0.773$ & $1.750,-1.145$ \\
\hline
\end{tabular}




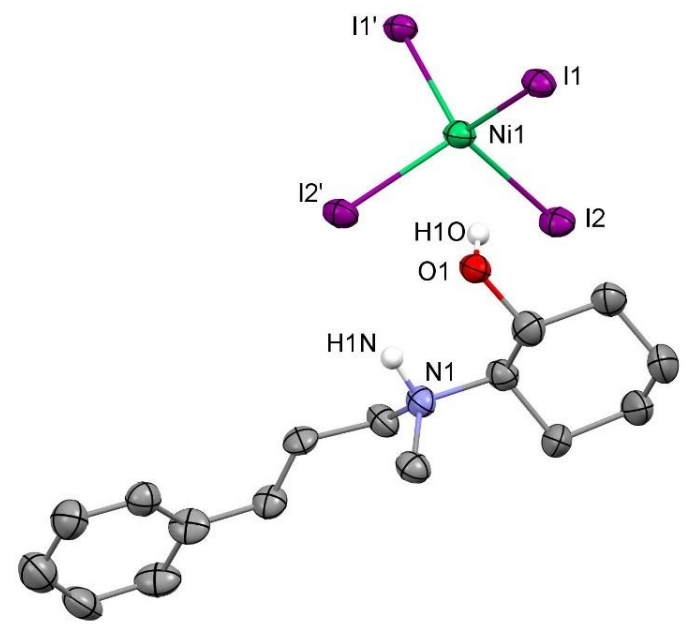

Figure S4. X-ray crystal structure of compound 4 with $50 \%$ probability ellipsoids. A cocrystallized benzene molecule and hydrogen atoms (except those on the alcohol and ammonium groups) are excluded for clarity.

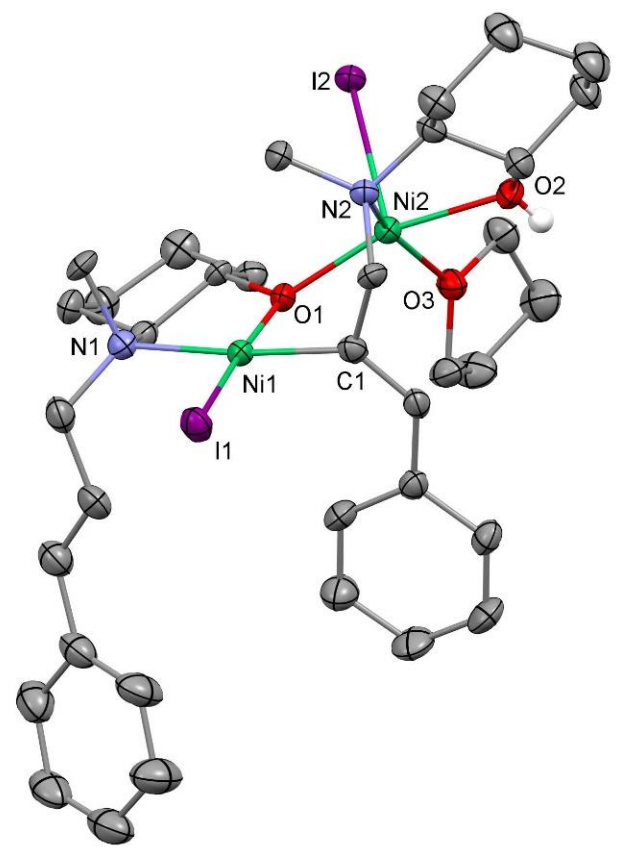

Figure S5. X-ray crystal structure of compound 5-THF with 50\% probability ellipsoids. Cocrystallized THF molecules and hydrogen atoms (except that on the alcohol group) are excluded for clarity. Selected bond distances $(\AA)$ and angles $\left(^{\circ}\right)$ : Ni1-I1 = 2.4936(9), Ni1-C1 = 1.890(6), Ni1-N1 = 2.043(5), Ni1-O1 = 1.886(4), Ni2-O1 = 1.988(4), Ni2-I2 = 2.6965(9), Ni2-N2 = 2.104(6), Ni2-O2 = 2.073(5), Ni2-O3 = 2.099(4), Ni1-O1-Ni2 = 111.9(2), O1-Ni1-N1 = 86.9(2), O1-Ni-C1 = 87.5(2), C1-Ni1-I1 = 87.65(19), I1-Ni1-N1 = 97.94(15), O2-Ni2-N2 = 80.3(2). 


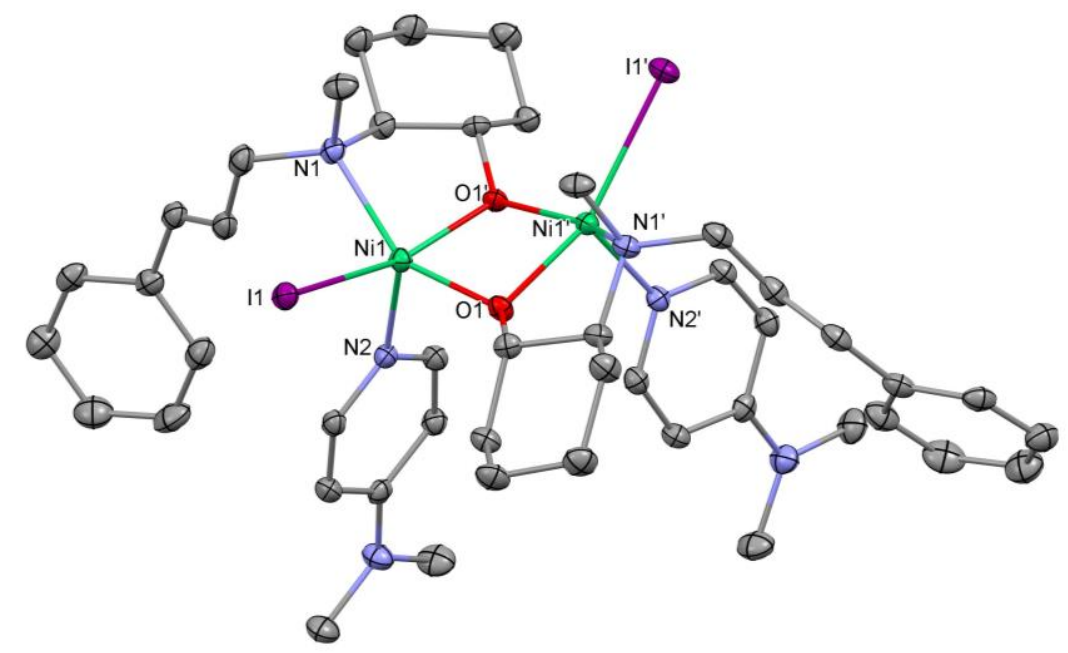

Figure S6. X-ray crystal structure of compound 6 with $50 \%$ probability ellipsoids. Cocrystallized benzene molecules and hydrogen atoms are excluded for clarity. Selected bond distances $(\AA)$ and angles $\left({ }^{\circ}\right)$ : Ni1-I1 = 2.7099(3), Ni1-O1 = 1.9863(14), Ni1-O1' $=1.9815(14)$, Ni1-N1 = 2.1649(18), Ni1-N2 = 2.0405(18), N1-Ni1-O1' = 80.66(6), Ni1-O1-Ni1' = 99.50(6).

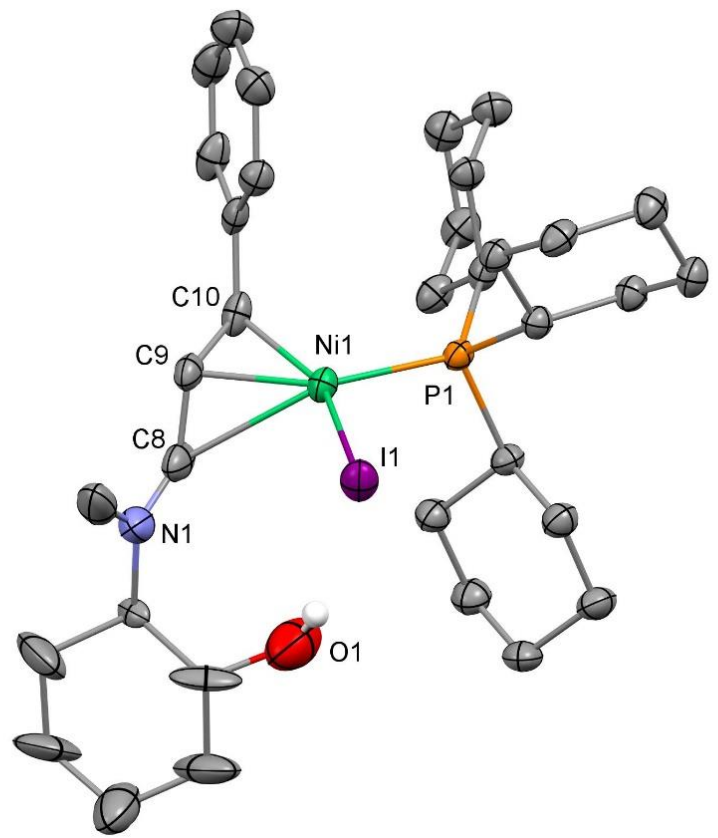

Figure S7. X-ray crystal structure of compound 7 with $50 \%$ probability ellipsoids. Hydrogen atoms (except that bound to the alcohol group) are excluded for clarity. Selected bond distances $(\AA)$ and angles $\left({ }^{\circ}\right)$ : Ni1-I1 = 2.5476(6), Ni1-P1 = 2.1812(11), Ni1-C8 = 2.473(4), Ni1-C9 = 2.004(4), Ni1-C10 = 1.959(4), P1-Ni1-I1 = 100.33(3). 


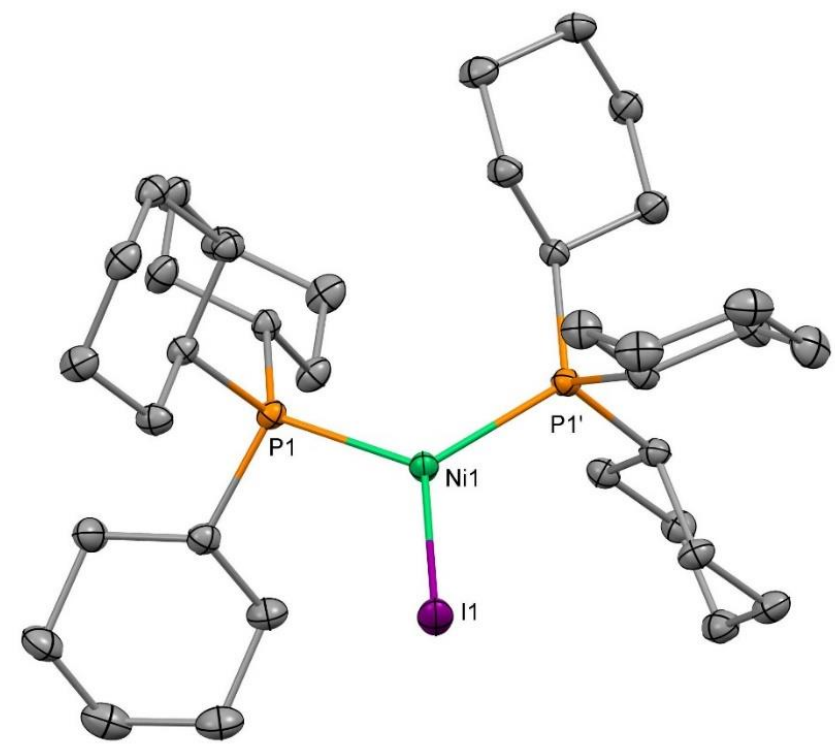

Figure S8. X-ray crystal structure of $\left(\mathrm{PCy}_{3}\right)_{2} \mathrm{NiI}$ with $50 \%$ probability ellipsoids. Hydrogen atoms are excluded for clarity. Selected bond distances $(\AA)$ and angles $\left({ }^{\circ}\right)$ : Ni1-I1 $=2.4841(6)$, Ni1-P1 = 2.2212(7), P1-Ni1-P1' = 118.45(4), P1-Ni1-I1 = 120.77(2).

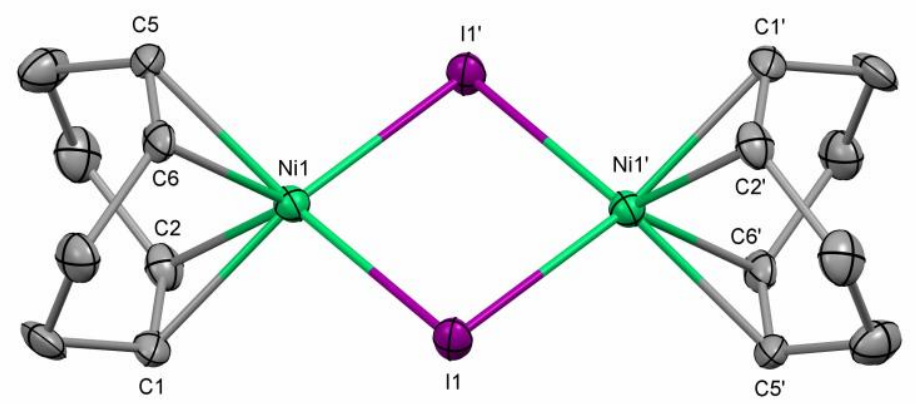

Figure S9. X-ray crystal structure of $[(\mathrm{COD}) \mathrm{NiI}]_{2}$ with $50 \%$ probability ellipsoids. Hydrogen atoms are excluded for clarity. Selected bond distances $(\AA)$ and angles $\left(^{\circ}\right)$ : Ni1-I1 $=2.6476(8)$, Ni1-I1' = 2.6313(8), Ni1-C1 = 2.145(5), Ni1-C2 = 2.114(4), Ni1-C5 = 2.149(4), Ni1-C6 = 2.118(4), C1-C2 = 1.363(6), C5-C6 = 1.361(6), Ni1-I1-Ni1' = 82.82(2), I1-Ni1-I1' = 97.18(2). 


\section{Computational Methodologies}

\section{General Notes}

All quantum mechanical calculations were carried out with the ORCA program. ${ }^{10}$ Geometry optimizations and harmonic frequency calculations were carried out with density functional theory (DFT). The PBE0 functional ${ }^{11}$ paired with Becke-Johnson damped D4 dispersion corrections $^{12}$, henceforth referred to as PBE0-D4, was used. Nickel is described by the def2-TZVP all electron basis set, ${ }^{13}$ while all other atoms are assigned the def2-TZVP(-f) basis. Diffuse functions are added to iodine (ma-def2-TZVP(-f)), as well as the ECP28MWB smallcore (25 explicit valence electrons) quasi-relativistic pseudopotential. ${ }^{14}$ This composite basis set is termed $\mathrm{BS} 1$. For reactions carried out in $\mathrm{MeCN}$, geometry optimizations and harmonic frequency calculations were conducted with the CPCM implicit solvation model $(\mathrm{MeCN}, \varepsilon=$ 36.6).${ }^{15}$ For all calculations employing CPCM, surface charges are described by the improved Gaussian charge scheme of Neese and coworkers with a scaled Van der Waals cavity $(\alpha=1.2) .{ }^{16}$ Corrections for outlying charge are included in solvated energies. Gas phase geometries are used for reactions carried out in benzene. For gas phase species, the nuclear Hessians were computed analytically, while numerical Hessians were employed for solvated complexes. Stationary points are characterized by the correct number of imaginary vibrational modes (zero for minima and one for saddle points). Intrinsic reaction coordinate (IRC) analysis confirms the nature of transition states. ${ }^{17}$ Cartesian coordinates of all optimized structures are included as ".xyz" files are available online in a compressed in a zip file format.

For complexes with the tricyclohexylphosphine ligand $\left(\mathrm{Cy}_{3} \mathrm{P}\right)$, the def2-TZVP and madef2-TZVP(-f) basis are used for nickel and iodine as mentioned above; however, the double- $\zeta$ split valence basis def2-SVP was employed for $\mathrm{C}, \mathrm{H}, \mathrm{O}, \mathrm{N}$, and $\mathrm{P}$. As these complexes contain up to $>140$ atoms, this is necessary for the computations to remain tractable. This mixed basis set is henceforth referred to as BS3.

Electronic energies are further refined with single point calculations employing the M06 functional ${ }^{18}$ and the def2-TZVPP basis set on all atoms with additional diffuse functions on I (ma-def2-TZVPP + ECP28MWB pseudopotential). This mixed basis is henceforth referred to as BS2. Solvation was accounted for with CPCM as mentioned above $(\mathrm{MeCN}, \varepsilon=36.6$; or benzene, $\varepsilon=2.28$ ). Final Gibbs free energies were obtained by applying thermodynamic 
corrections obtained at the optimization level of theory to these refined electronic energies. Thermodynamic corrections from harmonic frequency calculations employ the quasi-ridged rotor harmonic oscillator approach to correct for the breakdown of the harmonic oscillator approximation at low vibrational frequencies. ${ }^{19}$ Note that free energies are adjusted to a $1 \mathrm{M}$ standard state. Acetonitrile is defined to a standard state of $19.15 \mathrm{M}$ for reactions run in acetonitrile $\left(\Delta G^{0 \rightarrow *}=3.64 \mathrm{kcal} / \mathrm{mol}\right)$. The translational $\left(\mathrm{S}_{\text {trans }}\right)$ and rotational entropy $\left(\mathrm{S}_{\mathrm{rot}}\right)$ contributions to the Gibbs free energy calculated for a complex in condensed phase are $c a$. 40$60 \%$ of the values obtained assuming an ideal gas. ${ }^{20}$ As suggested in the literature, $S_{\text {trans }}$ and $S_{\text {rot }}$ obtained by ideal gas treatment are scaled by a factor of 0.5 to obtain the final condensed phase values. ${ }^{21}$ Hence, the final Gibbs free energy at $298.15 \mathrm{~K}$ is calculated as:

$$
G_{\text {solv }}^{*}=E_{e l, s o l v}^{M 06, B S 2}+Z P E+E_{\text {trans }}+E_{\text {rot }}+E_{\text {vib }}+k_{b} T-T\left(S_{e l}+S_{\text {vib }}+\frac{1}{2} S_{\text {trans }}+\frac{1}{2} S_{\text {rot }}\right)+\Delta G^{0 \rightarrow *}
$$

The resolution of identity (RI) and Chain-of-Spheres (COS) approximations are employed for efficient evaluation of Coulomb and exchange integrals, respectively. ${ }^{22}$ The def $2 / \mathrm{J}$ auxiliary basis ${ }^{23}$ is employed for all atoms except iodine, for which a suitable auxiliary was obtained via the automatic generation algorithm in the ORCA program (keyword: AutoAux). ${ }^{24}$ Very fine grid settings are employed in all calculations (optimization/frequency calculations: Grid6 NoFinalGrid GridX6, single point calculations: Grid7 NoFinalGrid GridX9).

Calculations on open-shell systems employ unrestricted Kohn-Sham orbitals (UKS). Spin contamination is generally minor. Open-shell singlets are modeled using the brokensymmetry (BS) formalism. For an open-shell singlet, construction of a proper spin eigenfunction of $\hat{S}^{2}$ cannot be achieved with a single Slater determinant. As such, BS-DFT does not properly describe the open-shell singlet as a pure singlet, but rather as a mixture of the corresponding singlet and triplet states. ${ }^{25}$ Various schemes for estimation of the singlet energy by removal of spin contamination have been developed. Here, we apply the formalism introduced by Yamaguchi and coworkers $^{26}$ :

$$
E_{S}=\frac{2\left(E_{B S}-E_{T}\right)}{\left\langle S^{2}\right\rangle_{T}-\left\langle S^{2}\right\rangle_{B S}}+E_{T}
$$

Along the minimum free energy path through the catalytic cycle as described in Figure 2 of the main text, high spin (triplet) $\mathrm{Ni}$ (II) intermediates were also considered. These were found to be higher in energy than their singlet counterparts (Figure S10). 


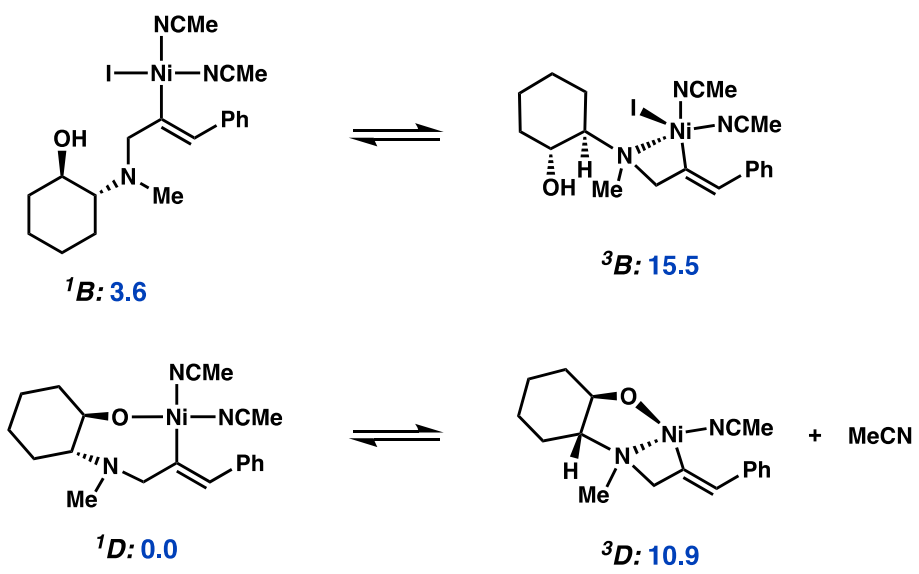

Figure S10. Relative free energies between singlet and triplet vinyl Ni(II) species.

\section{Nucleus Independent Chemical Shift (NICS) Calculations}

NICS calculations were carried out by DFT with the PBE0-D4 functional and def2-TZVP basis set in the gas phase. In accordance with literature convention, the reported NICS values are the negative of the total isotropic shift (in ppm) from the chemical shielding tensor. ${ }^{27}$ The NICS value is measured at the geometric center of the ring in question. As such, a negative NICS value - additional shielding at the ring center - is indicative of a diatropic ring current, hence indicating aromaticity. Analogously, positive NICS values are obtained for antiaromatic compounds. In the ORCA code, these calculations were accomplished by placing a ghost atom at the site of interest, assigning a single Gaussian s function (one primitive, exponent $=10^{6}$ ) with corresponding auxiliary to the ghost atom, and calculation of the NMR chemical shift (here using the gauge-independent atomic orbitals approach). 
Table S3. NICS (in ppm) at ring centers along C-O bond formation reaction coordinates. ${ }^{a}$

\begin{tabular}{|c|c|c|}
\hline Compound & NICS (ppm) & $\Delta \mathrm{NICS}(\mathrm{ppm})$ \\
\hline$F^{\text {phen }}$ & -7.19 & 0.00 \\
\hline TS(FG) $)^{\text {phen }}$ & -3.27 & +3.92 \\
\hline$G^{\text {phen }}$ & -6.91 & +0.28 \\
\hline$F^{\text {cin }}$ & -6.81 & 0.00 \\
\hline TS(FG) $)^{\text {cin }}$ & -5.02 & +1.79 \\
\hline$G^{\text {cin }}$ & -7.38 & -0.57 \\
\hline$F^{1 \text { nap }}$ & $\begin{array}{l}-7.94 \\
-8.07\end{array}$ & $\begin{array}{l}0.00 \\
0.00\end{array}$ \\
\hline TS(FG) $)^{1 \text { nap }}$ & $\begin{array}{l}-3.93 \\
-7.22\end{array}$ & $\begin{array}{r}+4.01 \\
+0.85\end{array}$ \\
\hline$G^{1 \text { nap }}$ & $\begin{array}{l}-5.59 \\
-7.80\end{array}$ & $\begin{array}{l}+2.35 \\
+0.27\end{array}$ \\
\hline$F^{2 \text { nap }}$ & $\begin{array}{l}-7.71 \\
-8.61\end{array}$ & $\begin{array}{l}0.00 \\
0.00\end{array}$ \\
\hline TS(FG) $)^{2 \text { nap }}$ & $\begin{array}{l}-4.68 \\
-4.80\end{array}$ & $\begin{array}{l}+3.03 \\
+3.81\end{array}$ \\
\hline $\mathbf{G}^{2 \text { nap }}$ & $\begin{array}{l}-5.90 \\
-8.07\end{array}$ & $\begin{array}{l}+1.81 \\
+0.54\end{array}$ \\
\hline benzene & -8.19 & - \\
\hline${\text { [benzene] }{ }^{2-}}^{-2}$ & $+7.80^{b}$ & - \\
\hline
\end{tabular}

${ }^{a}$ For naphthyl rings, second value in list is from second distal aryl ring. ${ }^{b}$ Dianion on neutral benzene geometry modeled with RKS for illustration purposes. $\triangle$ NICS defined to be NICS(transition state) NICS(starting complex). 


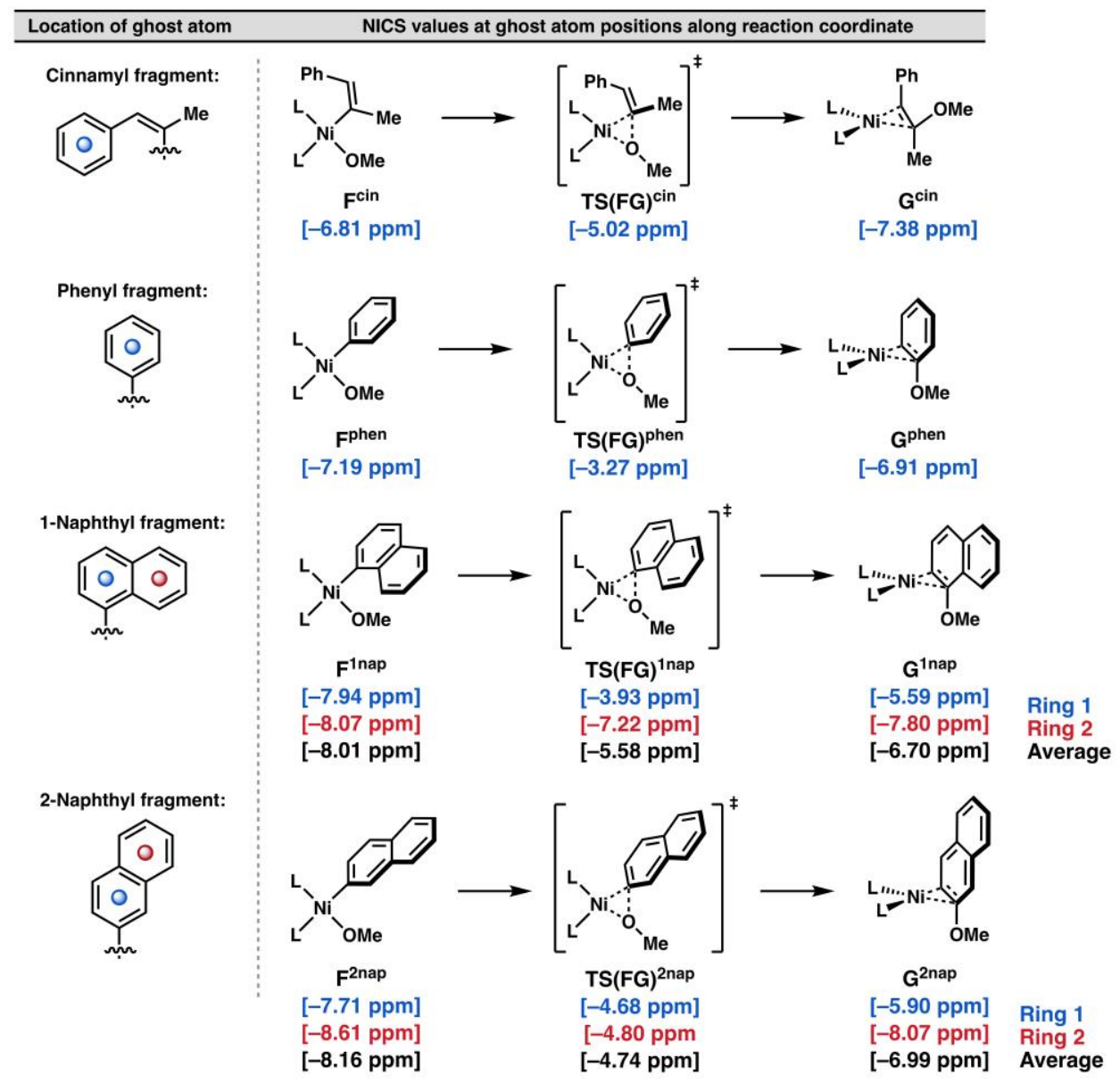

Figure S11. Schematic representation of NICS values (given in ppm) along reaction coordinate for $\mathrm{C}-\mathrm{O}$ bond forming pathways.

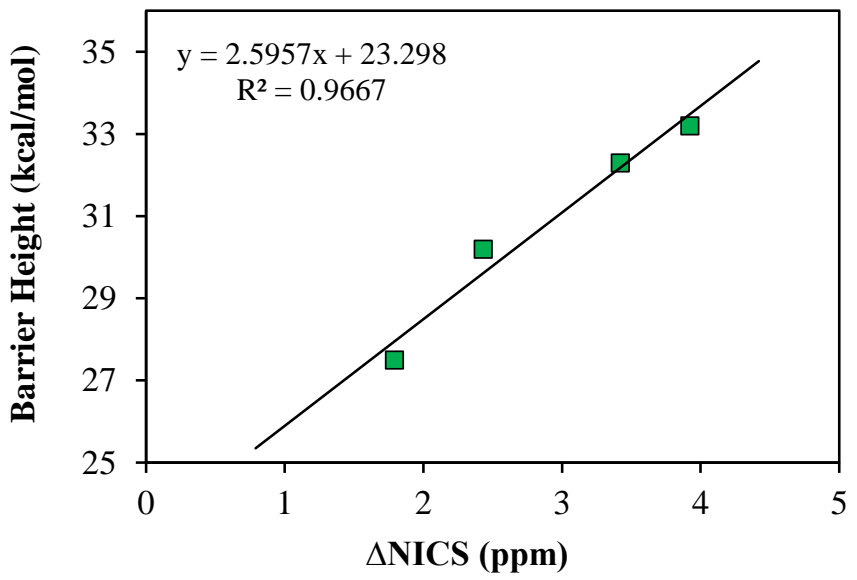

Figure S12. Plot of $\triangle$ NICS (ppm) versus calculated barrier height (kcal/mol). $\triangle$ NICS defined to be NICS(transition state) - NICS(starting complex). The $\triangle$ NICS value for naphthyl complexes is taken to be the average over the two aryl rings. 


\section{Coupled Cluster Calculations}

Domain-based local pair natural orbital (DLPNO)-CCSD(T) calculations were performed to obtain further refined electronic energies ${ }^{28}$ Coupled cluster (CC) calculations benefit from a degree of orbital relaxation of the reference determinant by the single orbital replacements from the $\mathrm{T}_{1}$ operator; however, the reference wavefunction should still provide a suitable basis for the coupled cluster expansion. ${ }^{29}$ We consider both canonical Hartree-Fock (HF) orbitals as well as Kohn-Sham (KS) orbitals as references. The approach of using non-HF orbitals as a reference basis has been demonstrated to be particularly advantageous when the HF determinant is a less ideal zeroth order approximation for the reference state - as is often the case with transition metal complexes. ${ }^{30}$ This effect has also been observed within the context of DLPNO-based CC calculations. ${ }^{31}$ In accord with literature findings, we observe the use of KS orbitals (BP86) in place of $\mathrm{HF}$ orbitals reduces the $\mathrm{T}_{1}$ diagnostic values ${ }^{32}$ in the $\mathrm{C}-\mathrm{O}$ bond forming transition states to generally $\leq 0.02$, compared to $>0.03$ as obtained with HF references. The range of the $\mathrm{T}_{1}$ diagnostic values with KS orbitals is reduced to 0.002 , compared to 0.011 with HF references. As such, KS (BP86) orbitals are preferred as the reference for DLPNO-CCSD(T) calculations presented herein. We note that qualitatively similar results are obtained with both HF and KS references.

We then turned our attention to the accuracy cutoff parameters in the DLPNO-based method ( $\mathrm{T}_{\text {CutPairs }}, \mathrm{T}_{\text {CutDO }}, \mathrm{T}_{\text {CutPNO}}$, and $\mathrm{T}_{\text {CutMKN }}$ ) as set by the keywords LoosePNO, NormalPNO, and TightPNO. While the NormalPNO settings capture the correct qualitative trends at affordable computational cost, we find deviations in barrier heights of up to $1.5 \mathrm{kcal} / \mathrm{mol}$ with respect to control calculations with the TightPNO settings (Table S4). As such, we elect to employ the TightPNO settings with BP86 reference orbitals for final energetics reported in this investigation. 
Table S4. Effect of choice of reference orbitals (HF or BP86) and PNO accuracy settings (Normal/Tight) on calculated barrier heights.

\begin{tabular}{|c|c|c|c|c|}
\hline Transition State & HF/Normal & HF/Tight & BP86/Normal & BP86/Tight \\
\hline TS(FG) ${ }^{\text {vin }}$ & $28.4[0.034]$ & $28.1[0.039]$ & $30.6[0.021]$ & $\mathbf{2 9 . 3}[\mathbf{0 . 0 2 1}]$ \\
\hline TS(FG) ${ }^{\text {cin }}$ & $30.0[0.028]$ & $28.5[0.033]$ & $28.1[0.021]$ & $\mathbf{2 7 . 5}[\mathbf{0 . 0 2 0}]$ \\
\hline TS(FG) ${ }^{\text {phen }}$ & - & $33.8[0.036]$ & - & $\mathbf{3 3 . 2}[\mathbf{0 . 0 2 0}]$ \\
\hline TS(FG) $)^{\text {1nap }}$ & - & $31.5[0.034]$ & - & $\mathbf{3 0 . 2}[\mathbf{0 . 0 1 9}]$ \\
\hline TS(FG) $)^{\text {2nap }}$ & - & $33.9[0.033]$ & - & $\mathbf{3 2 . 3}[\mathbf{0 . 0 1 9}]$ \\
\hline TS(FG) & - & $59.1[0.028]$ & - & $\mathbf{6 1 . 8}[\mathbf{0 . 0 1 9}]$ \\
\hline TS(DE) & $23.2[0.023]$ & $22.0[0.028]$ & - & $\mathbf{2 1 . 0}[\mathbf{0 . 0 1 9}]$ \\
\hline
\end{tabular}

$\mathrm{T}_{1}$ diagnostic provided in brackets next to barrier heights (given in $\mathrm{kcal} / \mathrm{mol}$ ).

In the current ORCA implementation, $\mathrm{CC}$ amplitudes are not self-consistently optimized with respect to the solvation SCRF. As a result, solvation is taken into account by the addition of $\Delta \mathrm{G}($ solv) calculated by DFT to gas phase $\mathrm{CC}$ electronic energies. $\Delta \mathrm{G}($ solv $)$ is calculated as $\mathrm{E}_{\mathrm{el}}(\mathrm{M} 06 / \mathrm{BS} 2 / \mathrm{CPCM}(\mathrm{MeCN}))-\mathrm{E}_{\mathrm{el}}(\mathrm{M} 06 / \mathrm{BS} 2)$. Hence, final Gibbs free energies are calculated as:

$$
G_{\text {solv }}^{*}=E_{\text {el,gas }}^{C C}+E_{\text {thermo }}^{D F T}+\Delta G_{\text {solv }}^{D F T}+\Delta G^{0 \rightarrow *}
$$

\section{Comparison of density functional methods in calculation of the barrier height of the rate- determining $\mathbf{C}-\mathbf{O}$ bond formation}

Taking the barrier obtained by DLPNO-CCSD(T)[TightPNO/BP86]/(ma)-def2TZVPP $+\Delta \mathrm{G}($ solv $)$ as a reference value $\left(\Delta \mathrm{G}^{\ddagger}=21.0 \mathrm{kcal} / \mathrm{mol}\right)$, we then investigated the results obtained via a variety of DFT methods for comparison. M06 ${ }^{18}$, a commonly employed functionals for evaluating energetics of transition metal containing systems, performs well with a $\Delta \mathrm{G}^{\ddagger}$ of $18.2 \mathrm{kcal} / \mathrm{mol}$. Less parameterized global hybrids $\left(\mathrm{B} 3 \mathrm{LYP}^{33}, \mathrm{PBE}^{11}\right)$ as well as HeadGordon's range-separated hybrid $\omega \mathrm{B} 97 \mathrm{M}-\mathrm{V}^{34}$ accordingly produce similar results. Surprisingly, we find $\mathrm{MN}^{35} 5^{35}$, parameterized to perform well across a variety of single and multi-reference systems, substantially underestimates barrier to $\mathrm{C}-\mathrm{O}$ bond formation $\left(\Delta \mathrm{G}^{\ddagger}=15.2 \mathrm{kcal} / \mathrm{mol}\right)$. 
Table S5. Comparison of DFT methods for computing barrier height for $\mathrm{C}-\mathrm{O}$ reductive elimination, TS(FG). ${ }^{a}$

\begin{tabular}{|c|c|}
\hline Method & Barrier height (kcal/mol) \\
\hline DLPNO-CCSD(T) & 21.0 \\
\hline M06 & 18.2 \\
\hline PBE0-D4 & 18.0 \\
\hline B3LYP-D4 & 20.9 \\
\hline$\omega B 97 M-V$ & 22.7 \\
\hline MN15 $^{b}$ & 15.2 \\
\hline
\end{tabular}

${ }^{a}$ Calculations performed on PBE0-D4/BS1/CPCM(MeCN) geometries. The def2-TZVPP basis set and $\mathrm{CPCM}(\mathrm{MeCN})$ models are used in the final single point calculations unless otherwise specified. ${ }^{b}$ Calculation performed using the Jaguar program with the def2-TZVPP(-g) basis set and PBF solvation model for MeCN.

\section{Notes on the Model System}

Stability of the RKS solution:

Conducting stability analysis on the RKS (PBE0/BS1/CPCM(MeCN)) wavefunction at the $\mathrm{C}-\mathrm{O}$ bond forming transition states reveals instability in some cases with respect to breaking of spin symmetry - favoring the UKS broken-symmetry (BS) solution, with $\left\langle S^{2}\right\rangle$ of up to 0.24. However, we find re-optimization along the broken-symmetry surface affords nearly identical geometries and final relative energies. Stability of the RKS density with respect to symmetry breaking is anticipated to be highly functional dependent. Control calculations with B3LYP-D4, M06, and PBE-D4 reveal minimal spin contamination and suggest the transition states are well described by a closed-shell KS determinant. 
Table S6. Value of $\left\langle S^{2}\right\rangle$ for broken-symmetry determinants (if exists) at the $\mathrm{C}-\mathrm{O}$ bond forming transition states.

\begin{tabular}{|c|c|c|c|c|}
\hline Transition State & PBE0-D4 & B3LYP-D4 & M06 & PBE-D4 \\
\hline $\mathrm{TS}(\mathrm{FG})^{\text {vin }}$ & 0.124 & 0.003 & 0.001 & 0.000 \\
\hline $\mathrm{TS}(\mathrm{FG})^{\text {cin }}$ & 0.000 & 0.000 & 0.000 & 0.000 \\
\hline $\mathrm{TS}(\mathrm{FG})^{\text {phen }}$ & 0.243 & 0.030 & 0.010 & 0.000 \\
\hline $\mathrm{TS}(\mathrm{FG})^{\text {1nap }}$ & 0.205 & 0.013 & 0.008 & 0.000 \\
\hline $\mathrm{TS}(\mathrm{FG})^{\text {2nap }}$ & 0.209 & 0.014 & 0.007 & 0.000 \\
\hline $\mathrm{TS}(\mathrm{FG})^{\mathrm{me}}$ & 0.000 & 0.000 & 0.000 & 0.000 \\
\hline
\end{tabular}

We became curious as to whether the stability of a broken-symmetry wavefunction is an artifact of the PBE0-D4 functional or an indicator of chemically relevant non-dynamical correlation. Plotting the $\alpha-\beta$ spin density for the PBE0-D4 broken-symmetry determinant of TS(FG) ${ }^{\text {phen }}$ reveals net $\alpha$ spin density on $\mathrm{Ni}$ (predominantly in the $\mathrm{d}_{\mathrm{x}-\mathrm{y}}{ }^{2}$ orbital), with a buildup of $\beta$ spin density in the $\pi$ system of the phenyl ring (Figure S13). Vertical excitation energies were evaluated with time-dependent DFT (TD-DFT) calculations (B3LYP-D4/def2TZVPP/CPCM(MeCN)) to check for low lying excited states (possibly involving the $\pi$ system of the vinyl/aryl substituents) in the vicinity of the transition state. However, the lowest vertical singlet excitations $\left[\mathrm{d}_{\mathrm{x}-\mathrm{y}}{ }^{2} / \pi^{*}\right.$ (aryl/vinyl) $\rightarrow \pi^{*} \mathrm{MeCN}$ ligand] occur from 1.6 to $>3.0 \mathrm{eV}$ for the six transition states, with the corresponding (spin-adapted) triplets $>1.0 \mathrm{eV}$ above the closedshell singlet ground state. Hence, the ground state singlet surface is well isolated from other excited states, particularly MLCT states, in the vicinity of the transition state, pointing to a suitable description within a single-reference framework. Similar TD-DFT results were obtained using the M06 functional. As discussed in the text, the aryl/vinyl $\pi / \pi^{*}$ systems are indeed involved in bond formation; however, this appears to be from the aforementioned $\sigma / \pi$ mixing rather than introduction of low-lying charge transfer excited states in the region of the TS. 


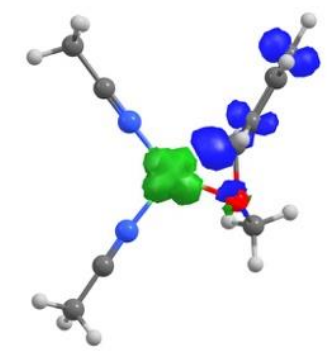

Figure S13. Plot of the $\alpha-\beta$ spin density for the PBE0-D4 broken-symmetry determinant of TS(FG) $)^{\text {phen }}$.

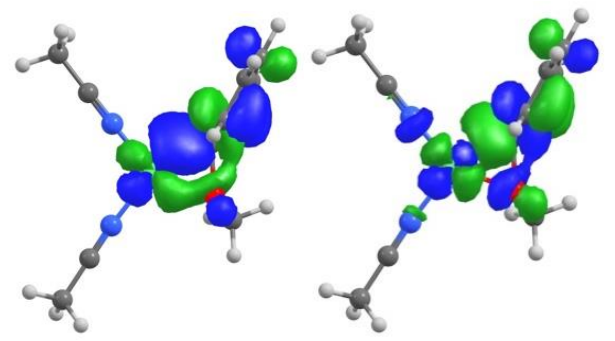

Figure S14. $\mathrm{Ni}-\mathrm{Ph} \sigma / \sigma^{*}$ pair included in the minimal $\mathrm{CAS}(2,2)$ active space.

To further probe this, multiconfigurational SCF calculations were carried out using complete active space self-consistent field (CASSCF) theory, including the bonding/antibonding orbital pairs as prompted by the broken-symmetry DFT calculations (Figure S14). The natural orbitals from the resulting $(2,2)$ active space mirror the $\mathrm{Ni} \mathrm{d}_{\mathrm{x}}{ }^{2}-\mathrm{y}{ }^{2} /$ phenyl $\pi^{*}$ character of the broken-symmetry calculations. The ground state configuration interaction (CI) vector is comprised of $84 \%$ of the [20] (doubly occupied bonding) and 16\% [02] (doubly occupied antibonding) configurations, indicating diradical character to be minimal. Moreover, CC calculations (vide supra) afford $T_{1}$ diagnostics of $\leq 0.021$, further suggesting an adequate single reference description.

In summary, these results suggest the dominance of a single configuration in the ground state, while highlighting the importance of the aryl/vinyl $\pi$ systems in the reductive elimination process. Moreover, we expect the DLPNPO-CCSD(T) calculations with TightPNO settings and BP86 reference orbitals are well suited for evaluating the energies at these transition states. 
Comparing CC to DFT in calculating barrier heights

Barrier heights calculated with M06 are systematically lower than those obtained with DLPNO-CCSD(T); however, the two methods show the same trends in reactivity (Table S7).

Table S7. DFT and CC barrier heights for $\mathrm{C}-\mathrm{O}$ reductive elimination.

\begin{tabular}{|c|c|c|}
\hline Transition State & M06 & DLPNO-CCSD(T) \\
\hline TS(FG) ${ }^{\text {vin }}$ & 25.4 & 29.3 \\
\hline $\mathrm{TS}^{\text {(FG }}{ }^{\text {cin }}$ & 24.9 & 27.5 \\
\hline $\mathrm{TS}(\mathrm{FG})^{\text {phen }}$ & 28.7 & 33.2 \\
\hline $\mathrm{TS}(\mathrm{FG})^{\text {1nap }}$ & 26.8 & 30.2 \\
\hline $\mathrm{TS}(\mathrm{FG})^{\text {2nap }}$ & 28.9 & 32.3 \\
\hline $\mathrm{TS}(\mathrm{FG})^{\mathrm{me}}$ & 53.8 & 61.8 \\
\hline
\end{tabular}

Calculations performed on PBE0-D4/BS1/CPCM(MeCN) geometries. The def2-TZVPP basis set and $\mathrm{CPCM}(\mathrm{MeCN})$ models are used in the final single point calculations. DLPNO-CCSD(T) calculations employ TightPNO settings with a BP86 reference as discussed above.

\section{C-H Bond Dissociation Energies as Metric for Disruption of $\boldsymbol{\pi}$ Bonding}

For $\mathrm{C}-\mathrm{O}$ bond formation via reductive elimination (TS(DE) and TS(FG)), the key role of $\sigma / \pi$ mixing at the transition state in facilitating bond formation is discussed in the main text. The degree of stabilization experienced at the transition state depends on the ability of the ligandbased $\pi^{*}$ to serve as an acceptor in the HOMO/LUMO mixing. For example, $\mathbf{F}^{\mathbf{v i n}}$ undergoes reductive elimination more readily than $\mathbf{F}^{\text {phen }}$, as partially occupying the phenyl $\pi^{*}$ is detrimental to the resonance stabilization of the $\pi$ system of the aryl ligand, whereas this does not apply to the vinyl $\pi$ system. Above, we investigate this from the viewpoint of NICS. Here, we the energetic cost of breaking a $\mathrm{C}=\mathrm{C}$ bond by virtue of the ability for a $\pi$ system to accept $\mathrm{H}^{*}$, generating the corresponding radical. We define $\mathrm{C}-\mathrm{H}$ bond dissociation energy (BDE) to be the change in electronic energy from the organic radical to the closed-shell $\pi$ system and $\mathrm{H}^{\bullet}$ (the reverse of the process stated above). A high $\mathrm{C}-\mathrm{H}$ BDE indicates less penalty for breaking the $\mathrm{C}=\mathrm{C}$ bond (Figure S15). A lower energetic penalty for lowering the bond order of the $\pi$ system would correlate with a more facile $\sigma / \pi$ mixing at the transition state (in which the ligand $\pi^{*}$ serves as an acceptor to a $\mathrm{M}-\mathrm{L} \sigma$ donor). As hypothesized, a correlation between $\mathrm{C}-\mathrm{H}$ BDE and barrier height for $\mathrm{C}-\mathrm{O}$ reductive elimination exists (Figure $\mathrm{S} 16$ ). 


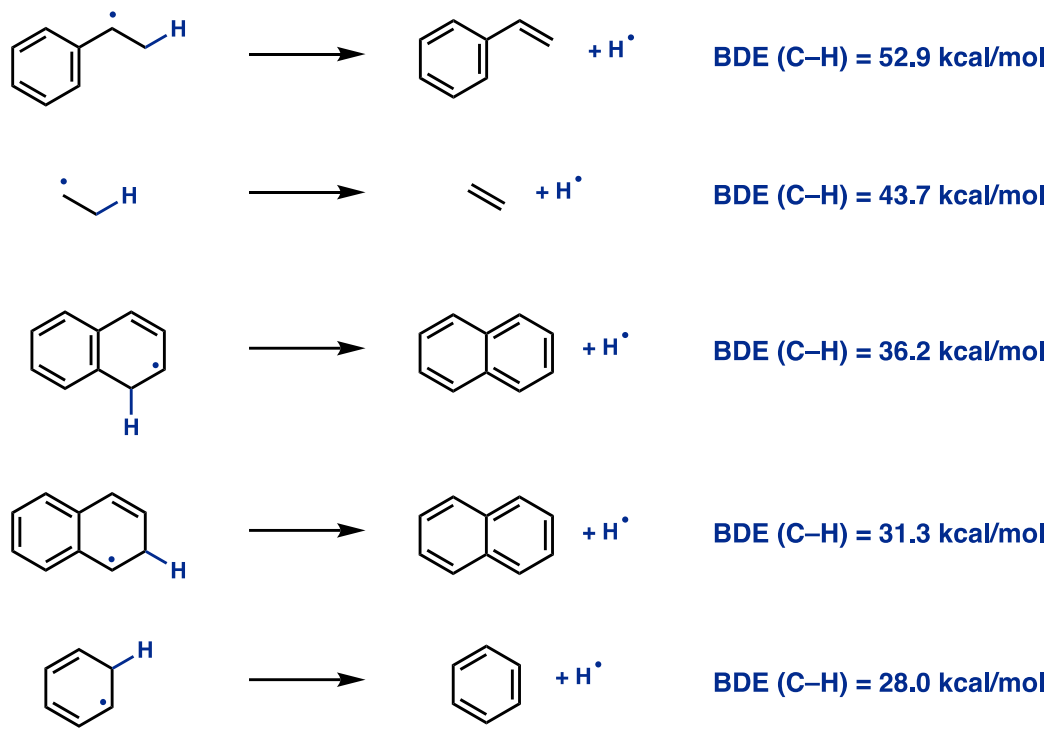

Figure S15. $\mathrm{C}-\mathrm{H}$ bond dissociation energy (BDE) from organic radical compounds (in $\mathrm{kcal} / \mathrm{mol})$. Calculated at the PBE0-D4/def2-TZVP(-f) level of theory.

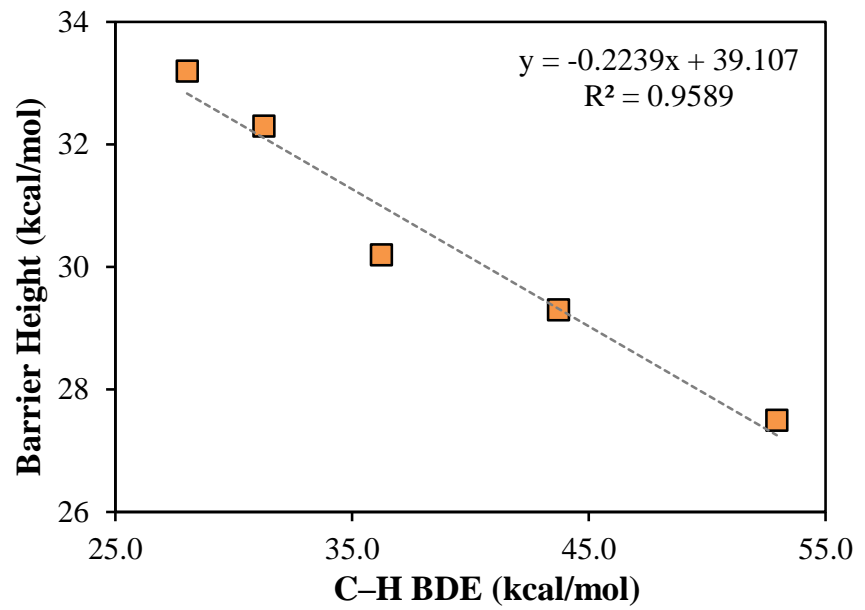

Figure S16. Plot of barrier height to $\mathrm{C}-\mathrm{O}$ reductive elimination from $\mathrm{Ni}(\mathrm{II})$ complexes $\mathbf{F}$ versus $\mathrm{C}-\mathrm{H}$ bond dissociation energy (BDE), in $\mathrm{kcal} / \mathrm{mol}$, from organic radical compounds corresponding to the $\mathrm{C}\left(\mathrm{sp}^{2}\right)$ fragments of complexes $\mathbf{F}$. 


\section{Oxidative Addition Mechanisms}

In the minimum free energy path presented in Figure 2 of the main text, four mechanisms of oxidative addition from $\mathbf{A}$ to $\mathbf{B} / \mathbf{C} / \mathbf{D}$ are considered ${ }^{36}$ : (1) A three-centered transition state leading to $\mathbf{B},(2)$ an anti-displacement-type mechanism leading to cationic $\mathrm{Ni}$ (II) intermediate $\mathbf{C}$, (3) halide abstraction affording a $\mathrm{Ni}(\mathrm{I})$ iodide and organic radical, and (4) outer-sphere electron transfer, followed by radical recombination (Figure S17). With an apparent barrier height of 10.3 $\mathrm{kcal} / \mathrm{mol}$, anti-displacement (TS(BC)) represents the lowest energy pathway for C-I bond cleavage. Oxidative addition via a canonical three-centered transition state is competitive, with a $\Delta \mathrm{G}^{\ddagger}$ of $13.5 \mathrm{kcal} / \mathrm{mol}$. As a control, geometries were also obtained in the gas phase. These calculations slightly favor the less polar three-centered transition state. Halide abstraction (TS(HI)) proceeds with a higher barrier of $22.8 \mathrm{kcal} / \mathrm{mol}$. Tris(acetonitrile) complexes were also considered.

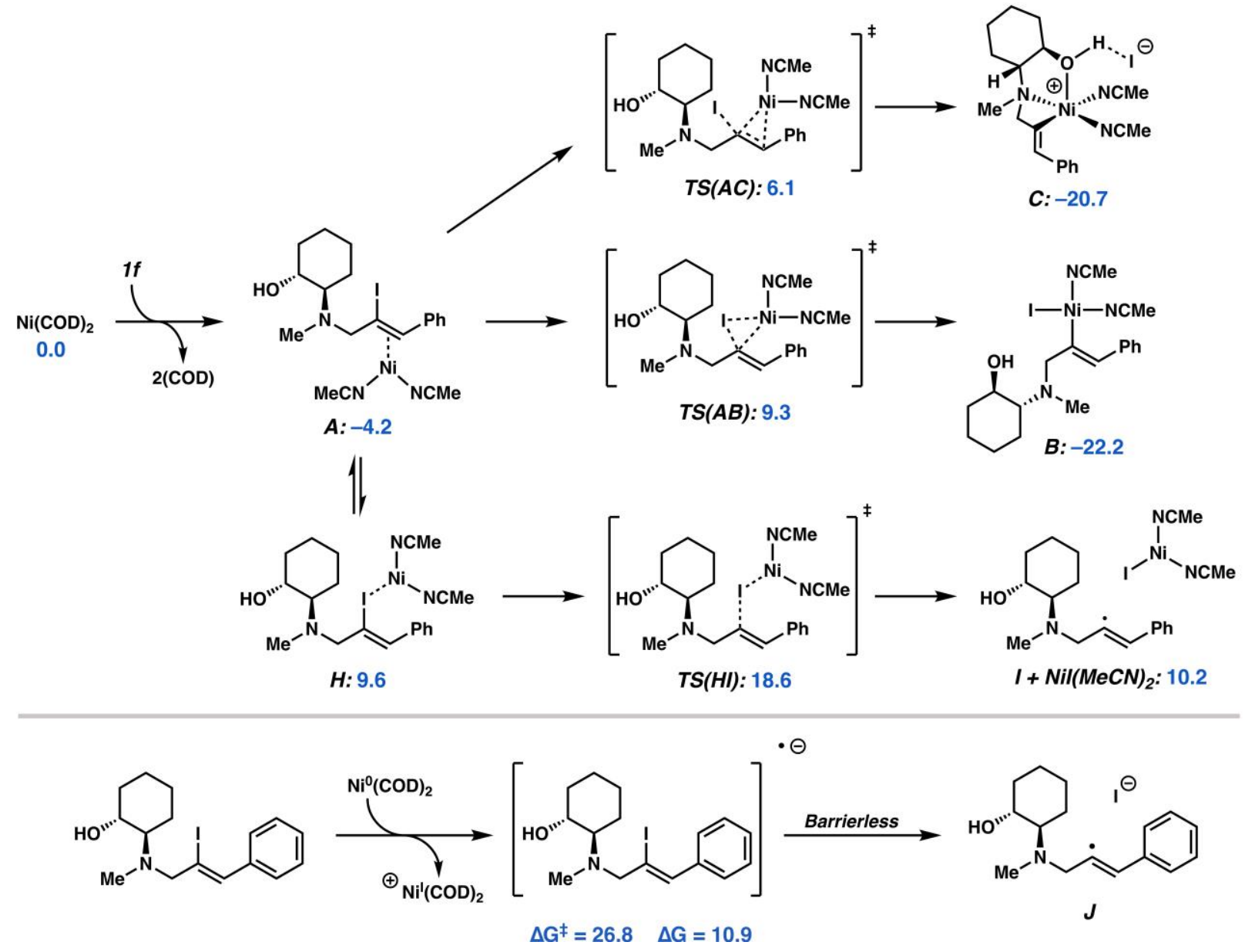

Figure S17. Oxidative addition mechanisms considered herein. 
The barrier height for outer-sphere electron transfer is evaluated by Marcus theory. Vertical reorganization energies $(\lambda)$ approximated by Nelsen's four-point model using electronic energies at the M06/BS2/CPCM(MeCN) level of theory (Figure S17). ${ }^{37}$ Hence, reorganization energy is calculated as the electronic energy of the initial state $(\mathbf{D}+\mathbf{A})$ at the optimized geometry of the initial state $(\mathbf{D}+\mathbf{A})$ subtracted from the electronic energy of the initial state $(\mathbf{D}+\mathbf{A})$ at the geometry of the final state $\left(\mathbf{D}^{{ }^{+}+\mathbf{A}^{-}}\right)$. Change in free energy $\left(\Delta G^{0}\right)$ is calculated at the M06/BS2/CPCM(MeCN)//PBE0-D4/BS1/CPCM(MeCN) level of theory. Note that single electron reduction of substrate $\mathbf{1 f}$ leads to concomitant cleavage of the $\mathrm{C}-\mathrm{I}$ bond to afford a $\mathrm{C}$ centered radical and iodide (Figure S17).

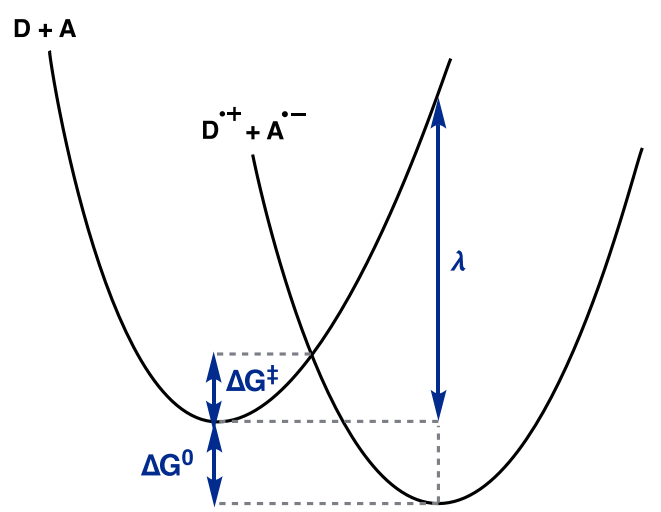

Figure S18. Model reaction coordinate for outer-sphere electron transfer.

The corresponding free energy barrier is calculated as:

$$
\Delta G^{\ddagger}=\frac{\left(\lambda_{0}+\Delta G^{0}\right)^{2}}{4 \lambda_{0}}
$$

A reorganization energy $(\lambda)$ of $83.9 \mathrm{kcal} / \mathrm{mol}$ paired with a reaction free energy $\left(\Delta G^{0}\right)$ of $10.9 \mathrm{kcal} / \mathrm{mol}$ affords $\Delta G^{\ddagger}$ of $26.8 \mathrm{kcal} / \mathrm{mol}$. 


\section{Thermodynamics of reorganization to $\pi$-allyl complex M}

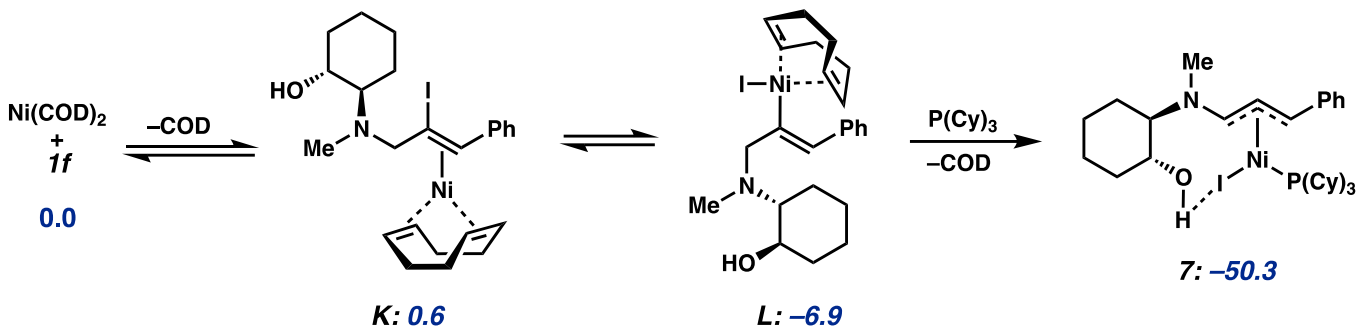

Figure S19. Free energy change for the reaction of $\mathrm{Ni}(\mathrm{COD})_{2}$ and substrate $\mathbf{1 f}$ to give $\pi$-allyl complex 7. Gibbs free energies give in $\mathrm{kcal} / \mathrm{mol}$ at the M06/(ma)-def2TZVPP/CPCM(Et $2 \mathrm{O}) / /$ PBE0-D4/BS3 level of theory.

In stoichiometric experiments, pre-mixing $\mathrm{PCy}_{3}$ and $\mathrm{Ni}(\mathrm{COD})_{2}$ followed by addition of $\mathbf{1 f}$ yields predominantly halide abstraction, producing $\left(\mathrm{PCy}_{3}\right)_{2} \mathrm{NiI}$ (see Figure 7 and 8 in the main text). We suspect this is due to the formation of sterically encumbered $\mathrm{PCy}_{3}$-ligated $\mathrm{Ni}(0)$ complexes which undergo halide abstraction with 1f preferentially to $2 \mathrm{e}^{-}$oxidative addition (see Figure 8 in main text). Instead, if substrate $\mathbf{1 f}$ is first reacted with 1 equivalent of $\mathrm{Ni}(\mathrm{COD})_{2}$ followed by addition of $\mathrm{PCy}_{3}, \pi$-ally complex 7 is formed. As such, we suspected that $\mathrm{Ni}(\mathrm{COD}) 2$ undergoes facile oxidative addition with $\mathbf{1 f}$ and the resultant $\mathrm{Ni}(\mathrm{II})$ vinyl iodide $\mathbf{L}$ is thermodynamically preferred to its $\mathrm{Ni}(0)$ precursors. Our computations corroborate this hypothesis, finding the formation of $\mathrm{Ni}$ (II) vinyl iodide $\mathbf{L}$ is accompanied by a free energy change of $-6.9 \mathrm{kcal} / \mathrm{mol}$ (Figure S19). In the absence of a vinyl iodide or base with zinc (II) salts, treatment of intermediate $\mathbf{L}$ with $\mathrm{PCy}_{3}$ affords $\pi$-allyl complex 7 with a $\Delta \mathrm{G}$ of $-50.3 \mathrm{kcal} / \mathrm{mol}$.

Despite extensive efforts, starting from the crystallographic structure of $\mathbf{7}$, optimizations at the PBE0-D4/BS3 level of theory return one small imaginary mode $\left(<100 \mathrm{~cm}^{-1}\right)$ corresponding to the rocking of the ally group/Ni center. Control optimizations and Hessian calculations with PBE-D4/BS3 level of theory afford nearly superimposable structures with no imaginary modes. Regardless, these observations bear no effect on the final conclusions.

\section{Disproportionation from $\mathrm{Ni}(\mathrm{II}) / \mathrm{Ni}(\mathrm{II})$ to $\mathrm{Ni}(\mathrm{I}) / \mathrm{Ni}(\mathrm{III})$}

We have further investigated the possibility for a disproportionation event between two $\mathrm{Ni}(\mathrm{II})$ species to give $\mathrm{Ni}(\mathrm{I})$ and $\mathrm{Ni}$ (III) intermediates. From the catalyst resting state (D) in $\mathrm{MeCN}$, outer-sphere electron transfer affords anionic $\mathrm{Ni}(\mathrm{I})$ and cationic $\mathrm{Ni}(\mathrm{III})$ intermediates $\mathbf{M}$ and $\mathbf{N}$, respectively. Geometric relaxation and solvent exchange afford a $\Delta \mathrm{G}$ of $41.5 \mathrm{kcal} / \mathrm{mol}$, 
disfavoring the formation of the $\mathrm{Ni}(\mathrm{I}) / \mathrm{Ni}(\mathrm{III})$ products (Figure S20). Although already 3.6 $\mathrm{kcal} / \mathrm{mol}$ higher in energy than the resting state (D), disproportionation from $\mathrm{Ni}$ (II) iodide complex B was also considered. Even allowing anion exchange for charge equilibration, disproportionation to $\mathrm{Ni}(\mathrm{I})$ and $\mathrm{Ni}(\mathrm{III})$ complexes $\mathbf{O}$ and $\mathbf{P}$ is disfavored by $53.6 \mathrm{kcal} / \mathrm{mol}$. Note that the lowest energy isomers and ligation environments are depicted. In summary, disproportionation from $\mathrm{Ni}(\mathrm{II}) / \mathrm{Ni}$ (II) to $\mathrm{Ni}(\mathrm{I}) / \mathrm{Ni}(\mathrm{III})$ is thermodynamically inaccessible. These results are in accord with prior literature. ${ }^{38}$
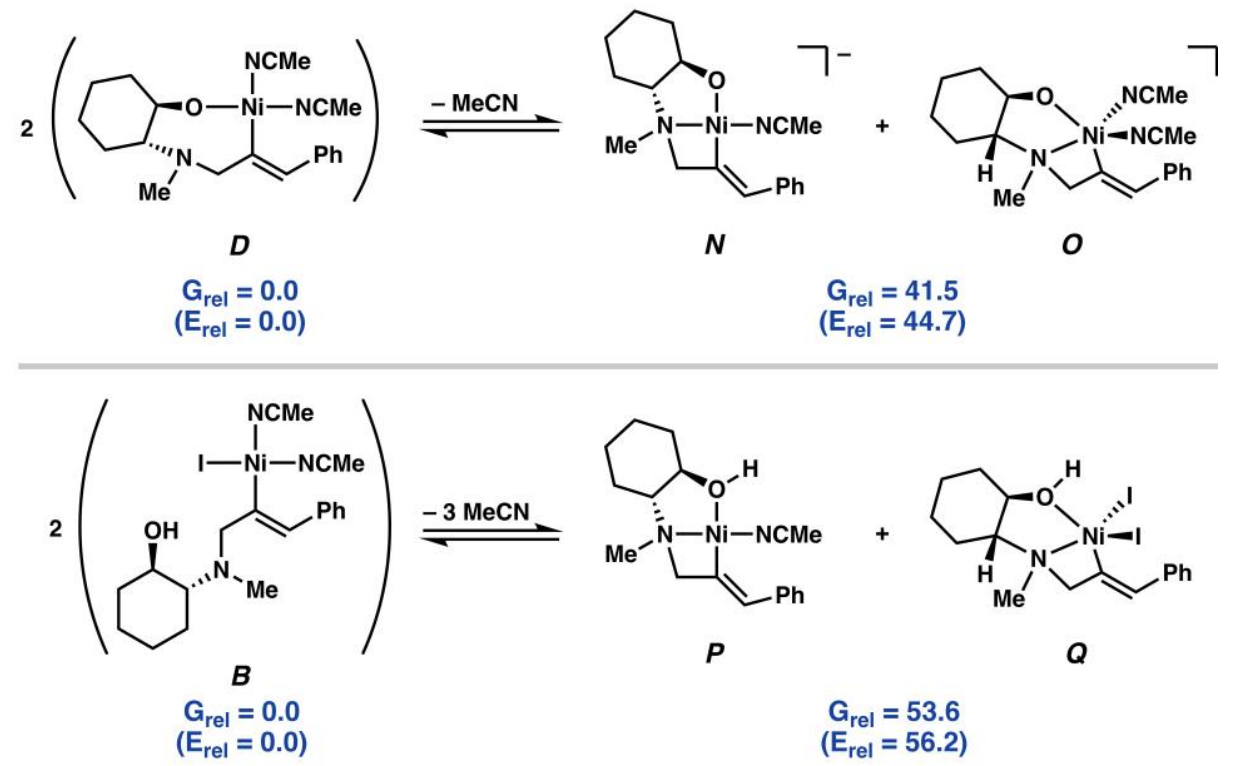

Figure S20. Thermodynamics of disproportionation of $\mathrm{Ni}(\mathrm{II}) / \mathrm{Ni}(\mathrm{II})$ to $\mathrm{Ni}(\mathrm{I}) / \mathrm{Ni}$ (III) from $\mathrm{Ni}$ (II) intermediates $\mathbf{B}$ and $\mathbf{D}$. Energies referenced from $2 \mathrm{~mol} \mathrm{Ni(II)}$ to $1 \mathrm{~mol} \mathrm{Ni(I)} \mathrm{plus} 1 \mathrm{~mol} \mathrm{Ni(III).}$

\section{Electronic structure of dinuclear Ni complex 5}

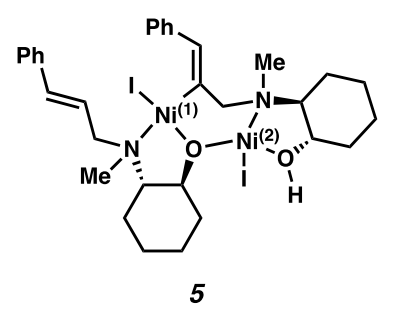

Complex $\mathbf{5}$ is a dinuclear nickel species bearing a square planar nickel center $\left(\mathrm{Ni}^{(1)}\right)$ and a distorted tetrahedral nickel $\left(\mathrm{Ni}^{(2)}\right)$ center. Given the ligand field environment, $\mathbf{5}$ was anticipated to present a triplet ground state with both nickel centers formally in the (II) oxidation state and with $\mathrm{Ni}^{(2)}$ in a high spin configuration. An effective magnetic moment ( $\mu_{\mathrm{eff}}$ ) of 3.57 B.M. (with 
diamagnetic correction) is found via Evan's method (see text). This is consistent with the presence of one high spin $\mathrm{Ni}$ (II) center. We sought to further explore the spin state assignment of 5 by computational analysis. The X-ray crystal structure of 5 contains a Ni-bound molecule of THF which hydrogen bonds to the pendant alcohol coordinated to $\mathrm{Ni}^{(2)}$. Elemental analysis of the bulk material suggests the THF molecule is indeed an artifact of subsequent crystallization conditions (THF/hexanes) employed for obtaining X-ray quality crystals. (Note that bulk $\mathbf{5}$ is isolated without exposure to THF). Indeed, when THF is present a $\Delta G$ of $1.0 \mathrm{kcal} / \mathrm{mol}$ is calculated favoring the formation of $\mathbf{5}$ 'THF from $\mathbf{5}$ and THF (Figure S21).
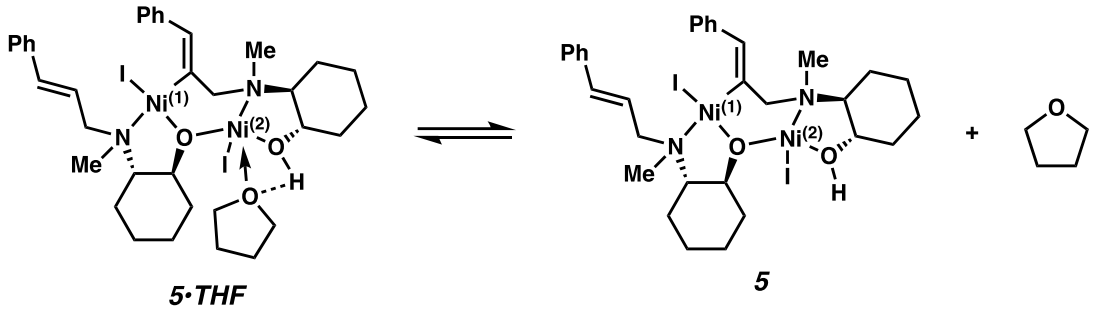

Figure S21. Coordination of THF to $\mathbf{5}$ to give $\mathbf{5} \cdot \mathbf{T H F}$ is favorable by $1.0 \mathrm{kcal} / \mathrm{mol}$.

To obtain a representative structure of $\mathbf{5}$, the Ni-bound molecule of THF was removed from the complex and the coordinates were relaxed. This was performed for the lowest energy closed-shell singlet (CSS), open-shell singlet (OSS), triplet, and quintet states. The optimization was carried out with DFT using the PBE0-D4 functional with the def2-TZVP basis set for Ni, ma-def2-SVP with ECP28MWB small-core pseudopotential for iodine, and def2-SVP for all other atoms. Single point calculations were then carried out on 5 at the M06/BS2/CPCM(benzene) level of theory for the lowest singlet, triplet, and quintet spin states. The triplet (low spin at $\mathrm{Ni}^{(1)}$ and high spin at $\mathrm{Ni}^{(2)}$ ) is lowest in energy with the CSS [two low spin $\mathrm{Ni}(\mathrm{II})$ ], OSS [antiferromagnetically-coupled high spin Ni(II) centers], and quintet [two high spin $\mathrm{Ni}(\mathrm{II}))]$ at 15.4, 13.9 and $13.9 \mathrm{kcal} / \mathrm{mol}$ higher in energy, respectively. While bond distances and angles vary, the ligation of $\mathbf{5}$ remained the same across the relaxed geometries in different electronic configurations. The coordinates for all four complexes are available in the coordinate data file, labeled as 5, 5_CSS, 5_OSS, and 5_HS. For high-spin Ni(II) centers, Löwdin spin populations of 1.603-1.570 were found (lower than the value of 2 from Ni-L covalency - the unpaired spin-bearing orbitals, $\mathrm{d}_{\mathrm{z}}{ }^{2}$ and $\mathrm{d}_{\mathrm{x}-\mathrm{y}}{ }^{2}$, have character on ligating atoms) (Table S8). Analysis of intrinsic atomic orbitals (IAOs) ${ }^{39}$ reveals $13 \alpha$ and $13 \beta$ spin electrons are localized 
to $\mathrm{Ni}^{(1)}$, while $14 \alpha$ and $12 \beta$ spin electrons are localized to $\mathrm{Ni}^{(2)}$. These results further corroborate the formal oxidation state assignments in complex $\mathbf{5}$ as two $\mathrm{Ni}(\mathrm{II})$ centers, $\mathrm{Ni}^{(1)}$ low spin and $\mathrm{Ni}^{(2)}$ high spin.

Table S8. Löwdin atomic spin populations for $\mathbf{5}$ optimized in different spin states. ${ }^{a}$

\begin{tabular}{|c|c|c|c|}
\hline Complex & $\begin{array}{c}\text { Löwdin spin } \\
\text { population on } \mathrm{Ni}^{(1)}\end{array}$ & $\begin{array}{c}\text { Löwdin spin } \\
\text { population on } \mathrm{Ni}^{(2)}\end{array}$ & $\left\langle\widehat{\boldsymbol{S}}^{2}\right\rangle$ \\
\hline 5 & 0.024 & 1.603 & 2.238 \\
\hline 5_CSS & 0.000 & 0.000 & 0.000 \\
\hline 5_OSS ${ }^{b}$ & 1.573 & -1.571 & 2.021 \\
\hline 5_HS & 1.566 & 1.570 & 6.023 \\
\hline
\end{tabular}

${ }^{a}$ Löwdin atomic spin populations and $\left\langle\widehat{\mathrm{S}}^{2}\right\rangle$ calculated with M06/BS2/CPCM(benzene). ${ }^{b}$ Net $\alpha-\beta$ signed spin density for an OSS is a consequence of the broken-symmetry formalism, i.e. the necessarily improper description of open-shell singlet states by a single Slater determinant. The opposite signed $\alpha-\beta$ spin density and each Ni center indicates the desired "OSS" state (brokensymmetry state) was captured, however we remind the reader that a $\alpha-\beta$ spin density for a proper singlet state is unphysical as a pure singlet state is defined as having $\left\langle\widehat{\mathrm{S}}^{2}\right\rangle=0$.

While these results suggest a ground state with formally $\mathrm{Ni}(\mathrm{II}) / \mathrm{Ni}(\mathrm{II})$ oxidation states, we were curious as to whether facile electron transfer could occur between the nickel centers to afford a Ni(I)/Ni(III) species. Time-dependent density functional theory (TD-DFT) calculations were carried out from the triplet ground state of 5 with the M06/BS2/CPCM(benzene) level of theory. The first 12 excitations $(0.48-2.00 \mathrm{eV})$ are $\mathrm{d}-\mathrm{d}$ excitations within the ligand field of single nickel centers. The first inter-nickel charge-transfer (CT) excitation found is at $2.16 \mathrm{eV}$ and is characterized by $\mathrm{CT}$ from $\mathrm{Ni}^{(1)} / \mathrm{L}$ to $\mathrm{Ni}^{(2)}$. Natural Transition Orbitals (NTOs) were utilized in this analysis. The NTO donor/acceptor pair describing $99 \%$ of the transition $(\mathrm{n}=$ 0.990) character is depicted below (Figure S22). Note that the donor NTO has significant character on the iodine ligand on $\mathrm{Ni}^{(1)}$. While the TD-DFT description of the energetics of CT states is often not quantitatively accurate, these initial calculations qualitatively highlight the significant preference for the $\mathrm{Ni}(\mathrm{II}) / \mathrm{Ni}(\mathrm{II})$ configuration over $\mathrm{Ni}(\mathrm{I}) / \mathrm{Ni}(\mathrm{III})$. 

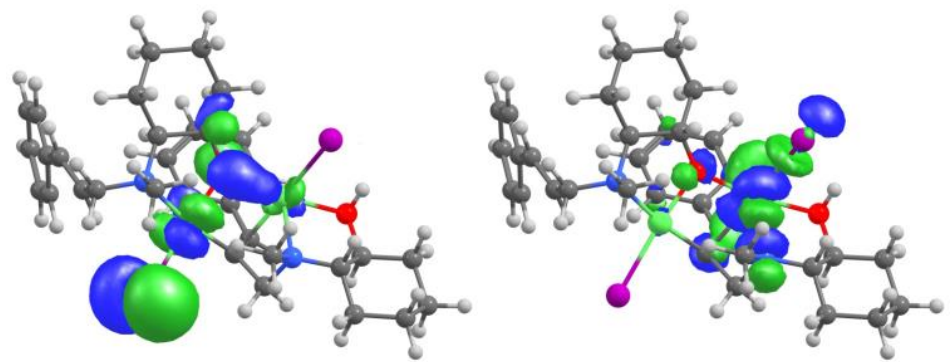

Figure S22. Donor (left) and acceptor (right) NTOs for $12^{\text {th }}$ root.

\section{Thermodynamics of the formation of complexes 6 and 7}

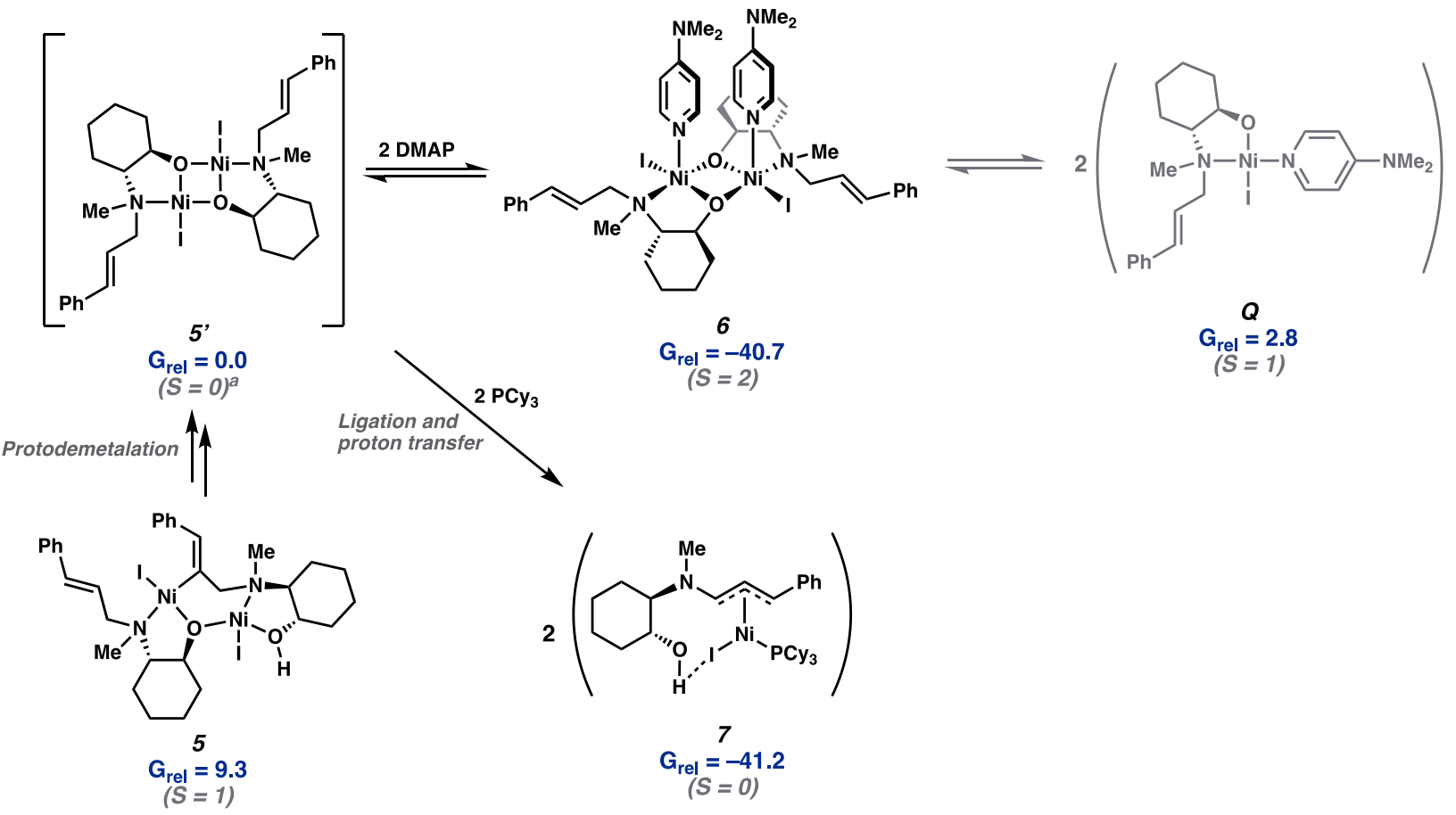

Figure S23. Relative free energies in $\mathrm{kcal} / \mathrm{mol}$ for dinuclear and mononuclear intermediates formed in non-coordinating solvents obtained as described above.

Complex 6 is high spin $(S=2)$ in the ground state. The open-shell singlet (antiferromagnetically-coupled high spin $\mathrm{Ni}$ (II) centers) and closed-shell singlet are found to be 3.7 and $30.3 \mathrm{kcal} / \mathrm{mol}$ higher in energy than the high spin complex. As with 5, the relaxed geometries for each spin state are available in the structure data file (6, 6_CSS, and 6_OSS).

Given the small difference in energy between the high spin and low spin states of 5', the assignment of spin state becomes increasingly dependent on the method employed. B3LYP-D4 and M06-L find the singlet and triplet state complexes to be less than $0.5 \mathrm{kcal} / \mathrm{mol}$ different in 
energy. PBE0-D4 affords a preference of $6.5 \mathrm{kcal} / \mathrm{mol}$ for the low spin 5' while M06 and TPSSh predict a closed-shell singlet by 8.2 and $8.3 \mathrm{kcal} / \mathrm{mol}$ (Table S9). These calculations highlight the known challenges of computing energy differences between different spin states in transition metal complexes. Regardless, we find the subsequent formation of DMAP and PCy 3 adducts 6 and 7 to be highly thermodynamically favorable and their formations from $\mathbf{5 / 5}$ ' irreversible.

Table S9. Comparison of relative energies of high-spin and low-spin 5' computed across different density functionals.

\begin{tabular}{|c|c|c|}
\hline Method & Low-spin R & High-spin R \\
\hline PBE0-D4 & 6.5 & 0.0 \\
\hline B3LYP-D4 & 0.0 & 0.3 \\
\hline M06-L & 0.0 & 0.4 \\
\hline M06 & 0.0 & 8.2 \\
\hline TPSSh & 0.0 & 8.3 \\
\hline
\end{tabular}

\section{Acid/Base equilibrium and importance of $\mathrm{Zn}(\mathrm{II})$ salt additives}

Throughout our experimental and computational investigations, we find that $\mathrm{Zn}$ (II) salts play an important role in sequestering iodide produced after oxidative addition. These may be produced either from reduction of sacrificial Ni iodide species or from the reaction of $\mathrm{Zn}^{0}$ with the $\mathrm{HI}$ formed in the reaction after oxidative addition. Modeling the exact speciation of $\mathrm{Zn}$ (II) salts in the reaction is challenging and this matter is further complicated by (in)solubility of some of these potential adducts. Likewise, $\mathrm{Zn}(\mathrm{OTf})_{2}$ is used in the modified reaction conditions due to its improved solubility over $\mathrm{ZnI}_{2}$. For simplicity, we highlight in a general case how solvated $\mathrm{ZnI}_{2}$ may aid in sequestration of iodide through formation of zinc-ate $\left(\mathrm{ZnI}_{4}{ }^{2-}\right)$ derived intermediates. The computed $\Delta \mathrm{G}$ of $-29.5 \mathrm{kcal} / \mathrm{mol}$ for formation of $\left[\mathrm{Et}_{3} \mathrm{NH}\right]_{2} \mathrm{ZnI}_{4}$ from $\mathrm{ZnI}_{2}$ and $\mathrm{Et}_{3} \mathrm{NHI}$ demonstrates this point (Figure S24). The corresponding process with $\mathrm{Zn}(\mathrm{OTf})_{2}$ should be similar, if not even more favorable. 


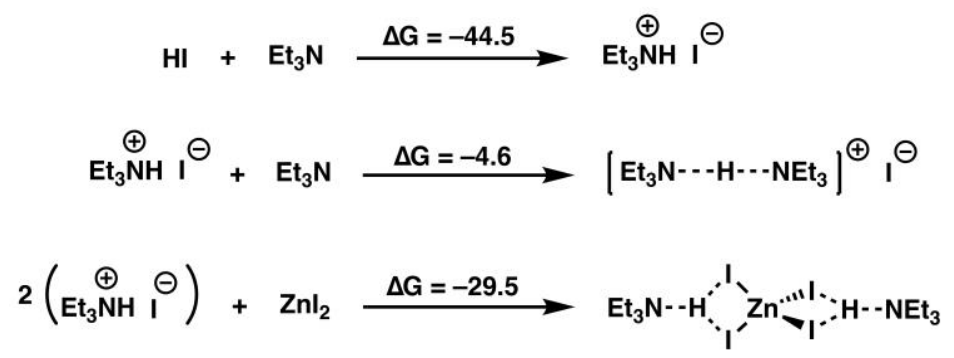

Figure S24. Impact of $\mathrm{ZnI}_{2}$ on sequestration of iodide and formation of stable adducts.

\section{Alternative $\mathrm{C}-\mathrm{O}$ bond forming mechanistic hypotheses}

Throughout the course of our investigation numerous mechanistic hypotheses were considered for $\mathrm{C}-\mathrm{O}$ bond formation. While in the full context of experimental and computational investigation it is now clear that $\pi$-allyl complex $\mathbf{7}$ is not catalytically relevant, we initially suspected $\mathrm{C}-\mathrm{O}$ bond formation may occur from nucleophilic attack of the electrophilic $\beta$ carbon of the $\pi$-allyl moiety (Figure S25). Our initial investigations highlight a thermodynamic preference of $13.8 \mathrm{kcal} / \mathrm{mol}$ for $\pi$-allyl intermediate $\mathbf{R}$ over metallacyclobutane $\mathbf{S}$. Critically, given the geometric constraints of $\mathbf{S}, \beta$-hydride elimination is not anticipated to be facile. Accordingly, a barrier of $32.0 \mathrm{kcal} / \mathrm{mol}$ is found for this step (TS(ST)). Note that high spin $\mathbf{S}$ was found to have a relative free energy of $39.8 \mathrm{kcal} / \mathrm{mol}$. These preliminary results we obtained with the PBE-D4/def2-TZVP [Ni], ma-def2-SV(P) [I], def2-SV(P) level of theory.
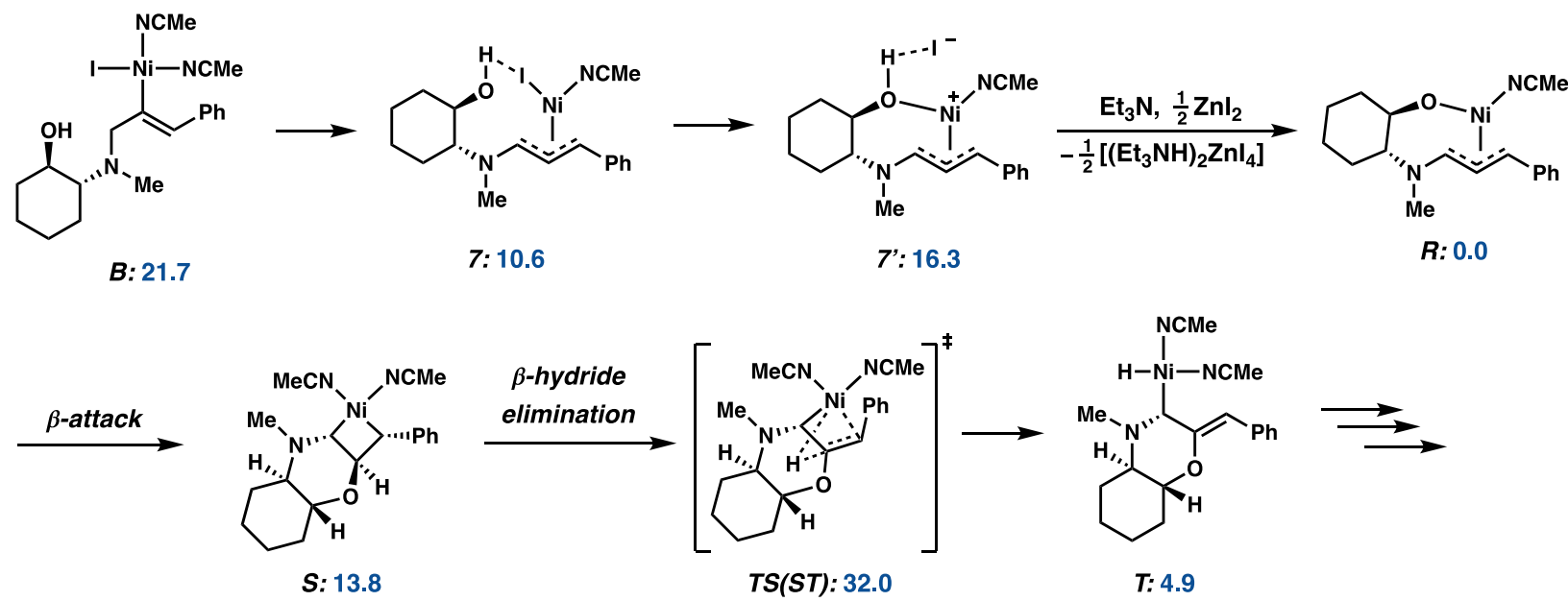

Figure S25. Alternative mechanistic hypothesis for $\mathrm{C}-\mathrm{O}$ bond formation. Relative free energies given in $\mathrm{kcal} / \mathrm{mol}$. 
An oxa-Heck type mechanism was also considered starting from a protodemetalated olefin. Syn-addition of a nickel alkoxide can be eliminated on a basis of stereochemical outcome of the observed product - the enol ether after subsequent $\beta$-hydride elimination would bear the $E$ olefin geometry when starting from the $Z$ styrene. The charge separated intermediates required for an anti-addition are found to be high in energy or unstable on the PES. When olefin 3 is subjected to the reaction conditions (with or without strong exogenous base), no productive reaction is experimentally observed. 
NMR Spectra of New Compounds and Key Experiments

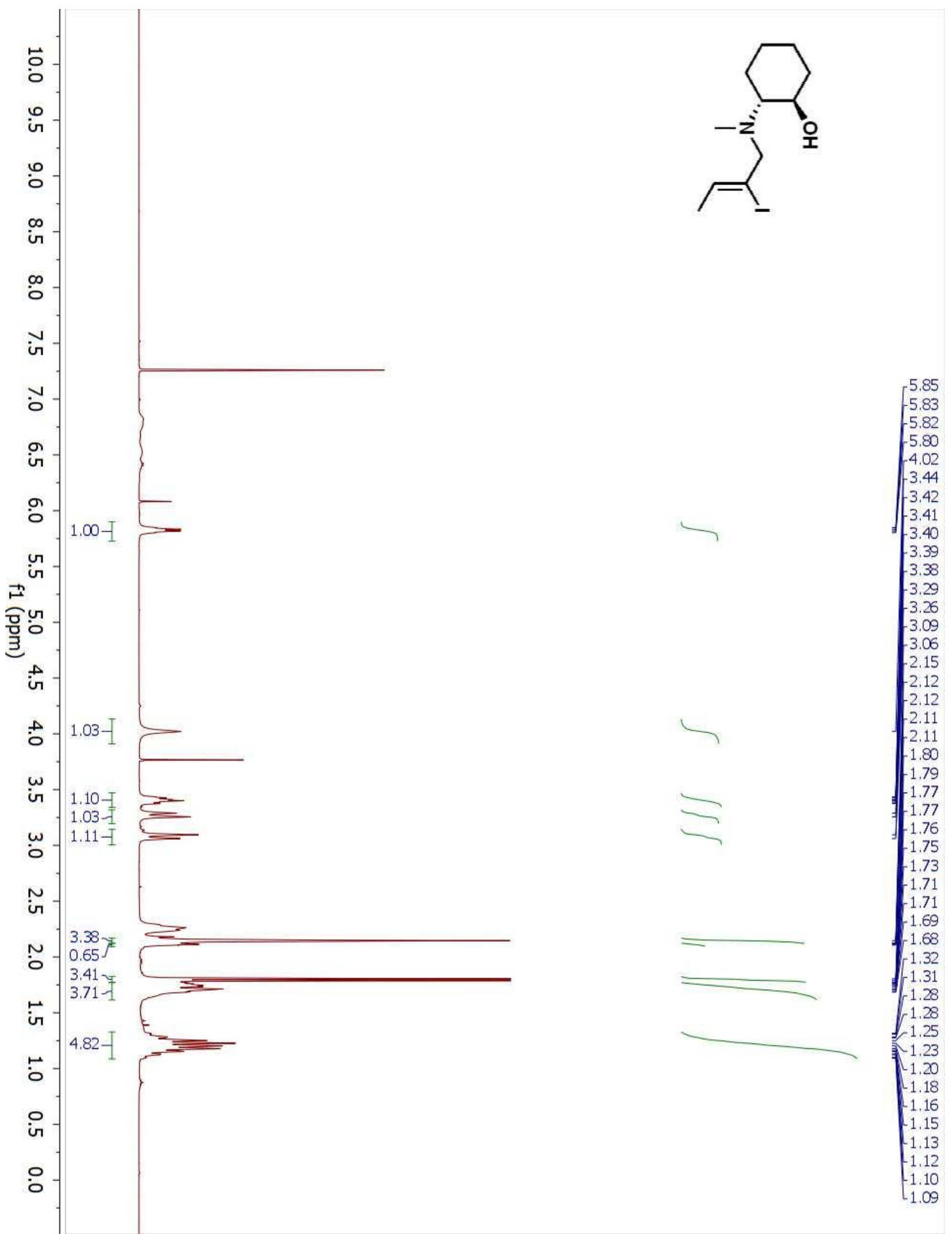

Figure S26. ${ }^{1} \mathrm{H}$ NMR spectrum of $\mathbf{1 a}$ in $\mathrm{CDCl}_{3}$. 


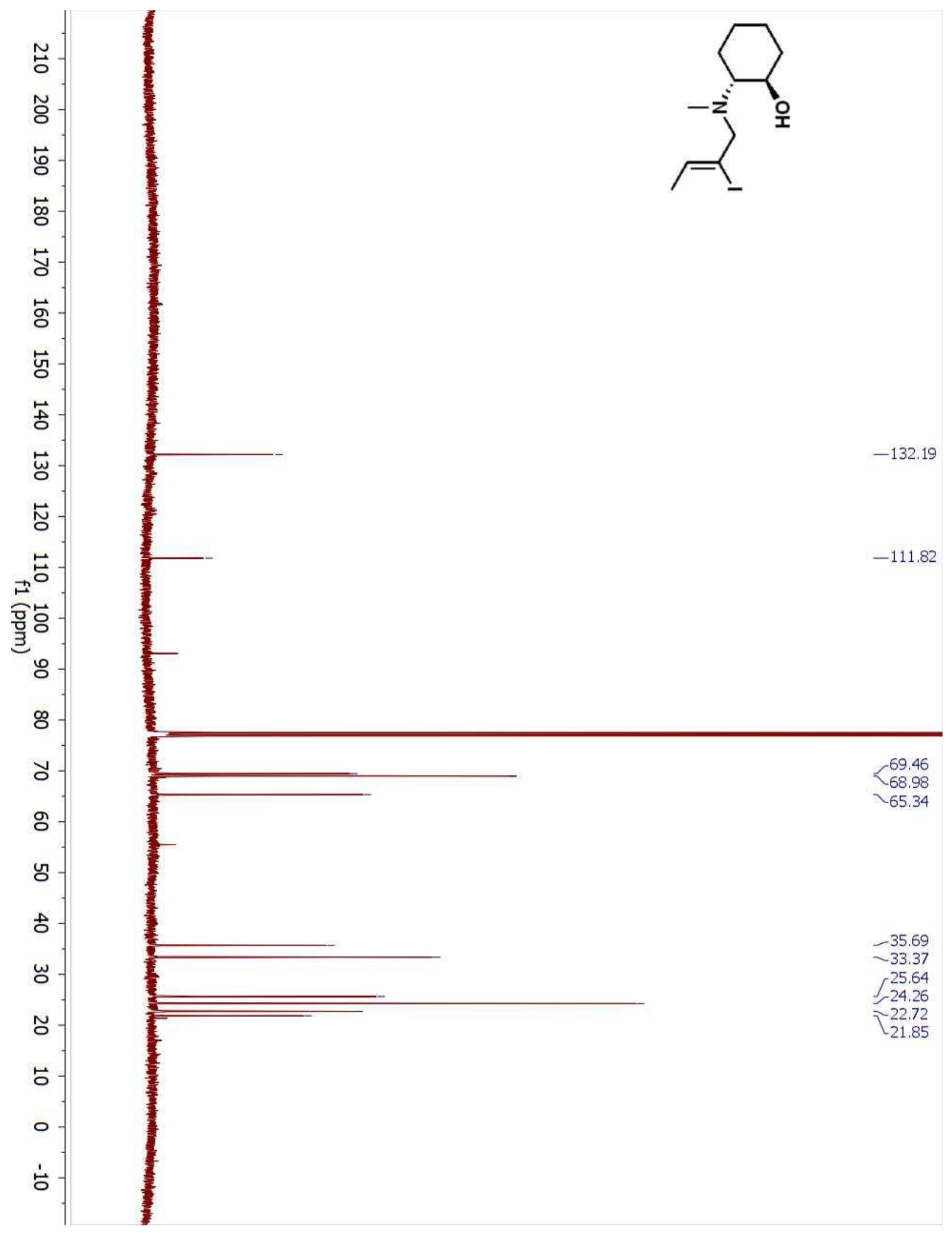

Figure S27. ${ }^{13} \mathrm{C}$ NMR spectrum of $1 \mathbf{a}$ in $\mathrm{CDCl}_{3}$. 


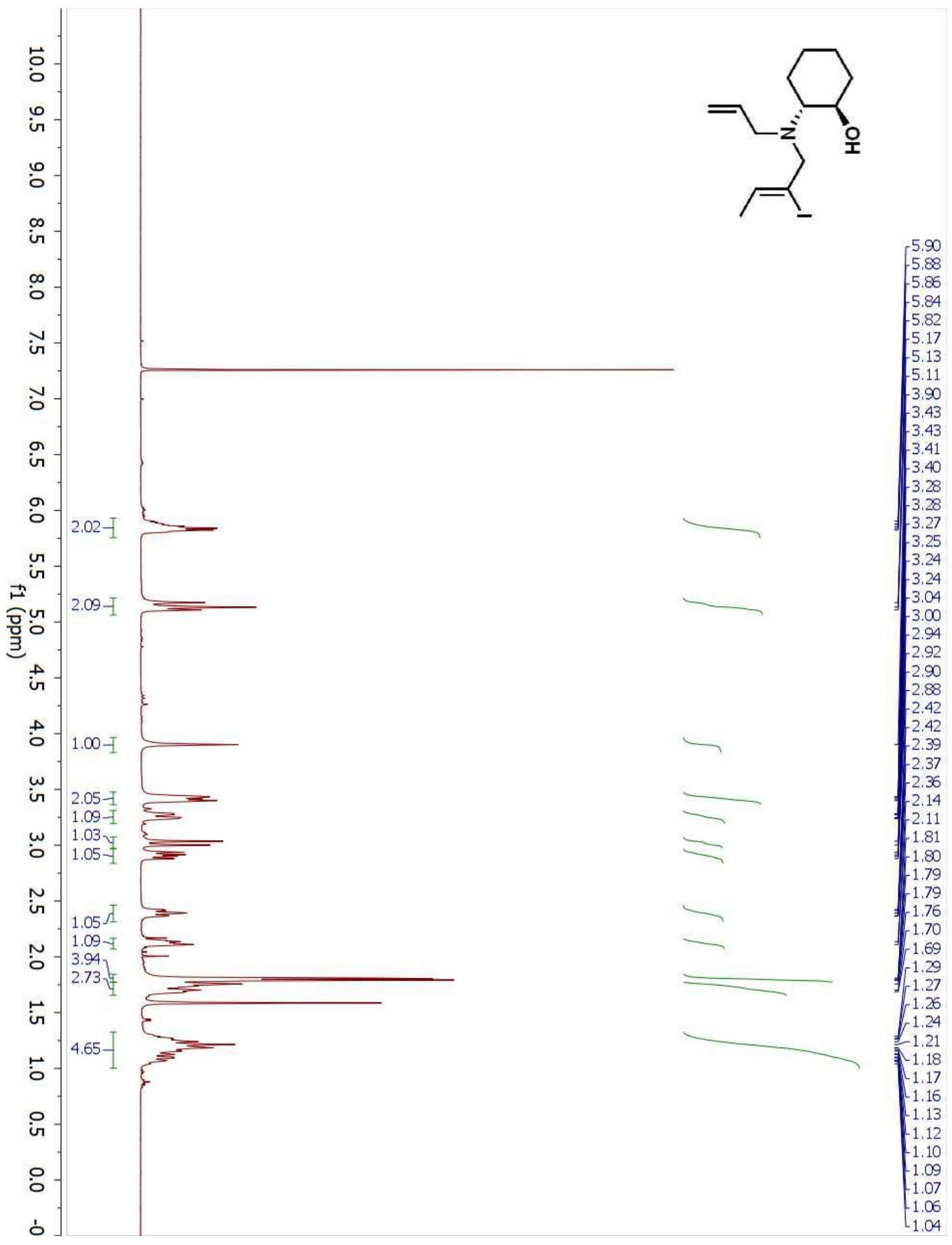

Figure S28. ${ }^{1} \mathrm{H}$ NMR spectrum of $\mathbf{1 d}$ in $\mathrm{CDCl}_{3}$. 


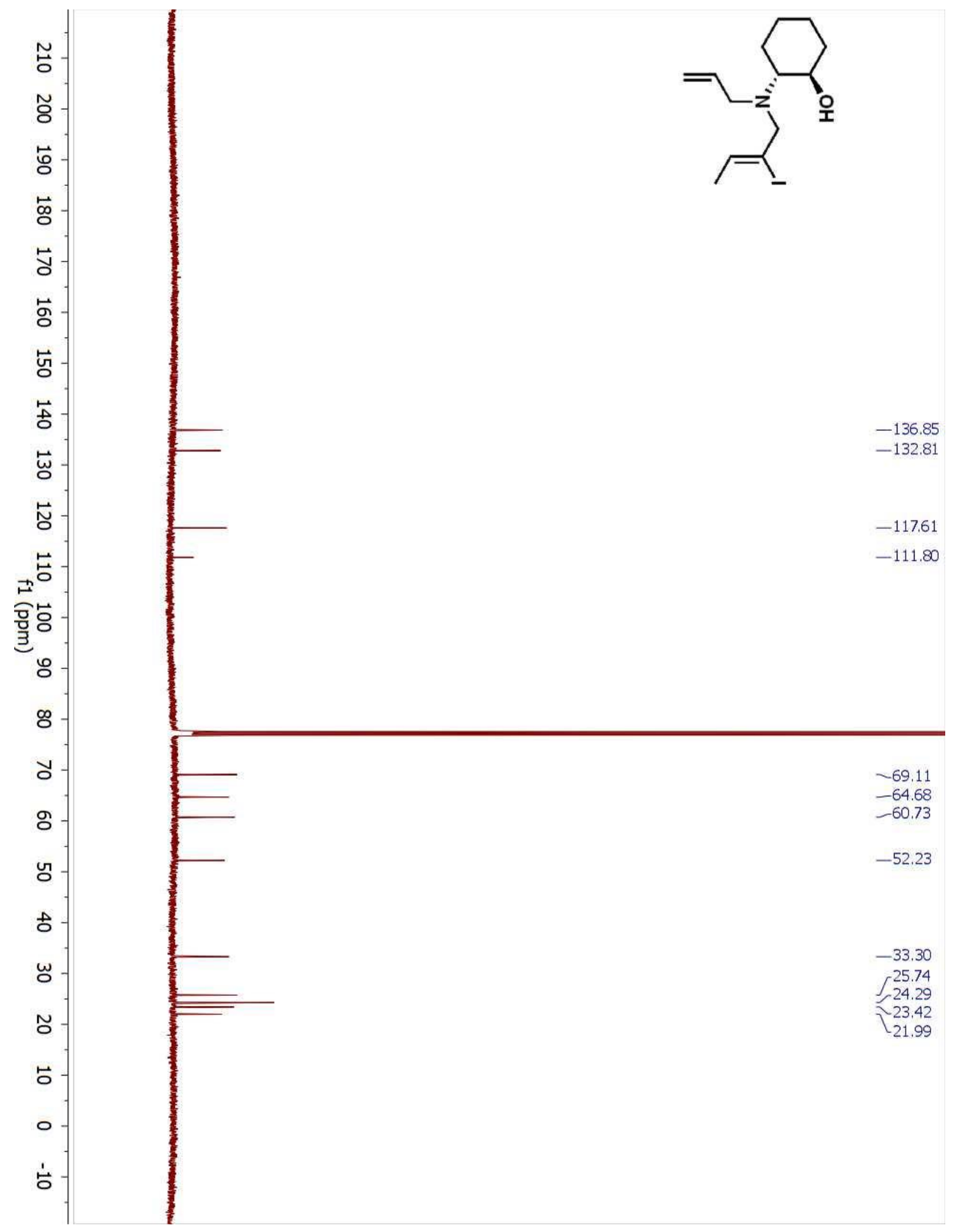

Figure S29. ${ }^{13} \mathrm{C}$ NMR spectrum of $\mathbf{1 d}$ in $\mathrm{CDCl}_{3}$. 


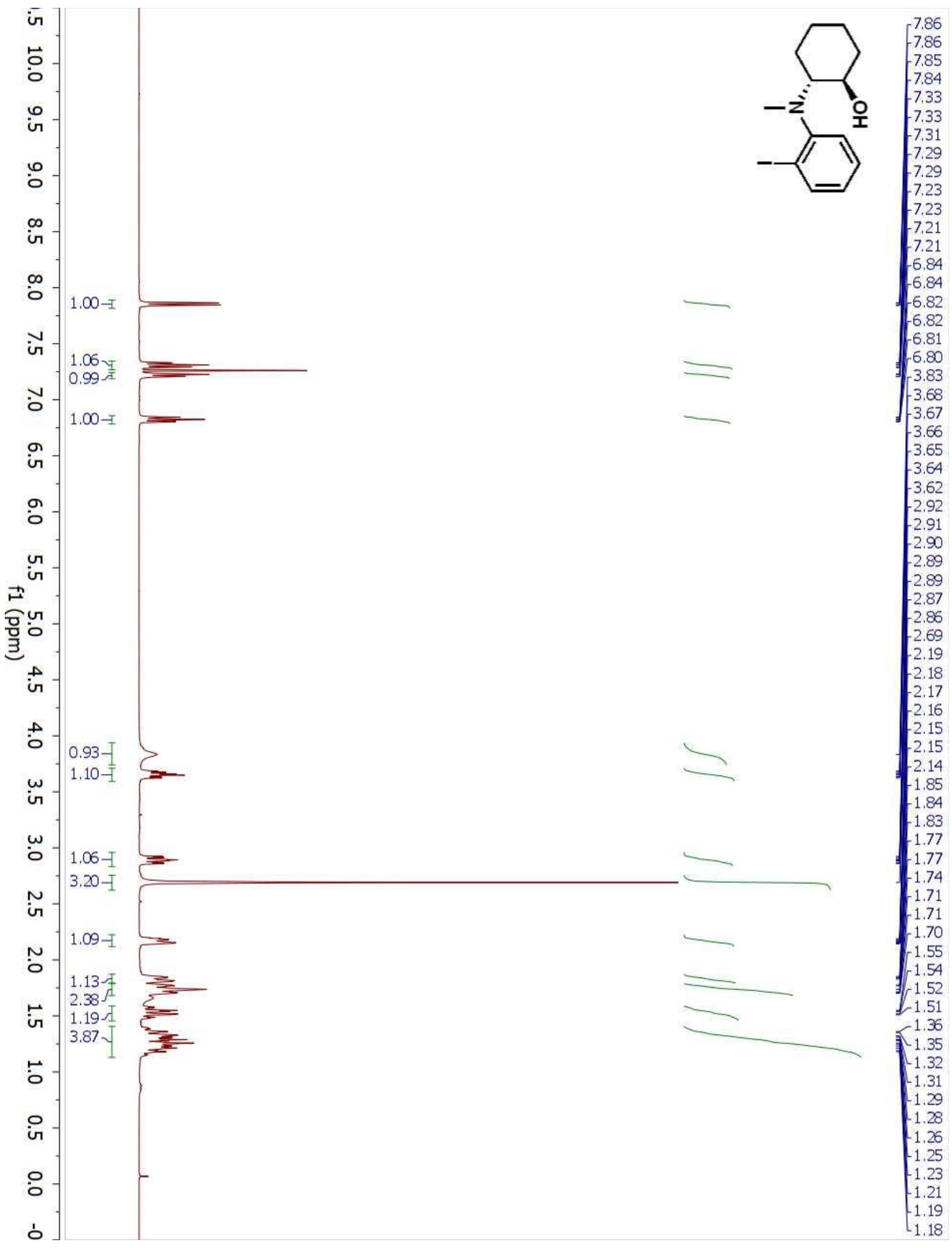

Figure S30. ${ }^{1} \mathrm{H}$ NMR spectrum of $1 e$ in $\mathrm{CDCl}_{3}$. 


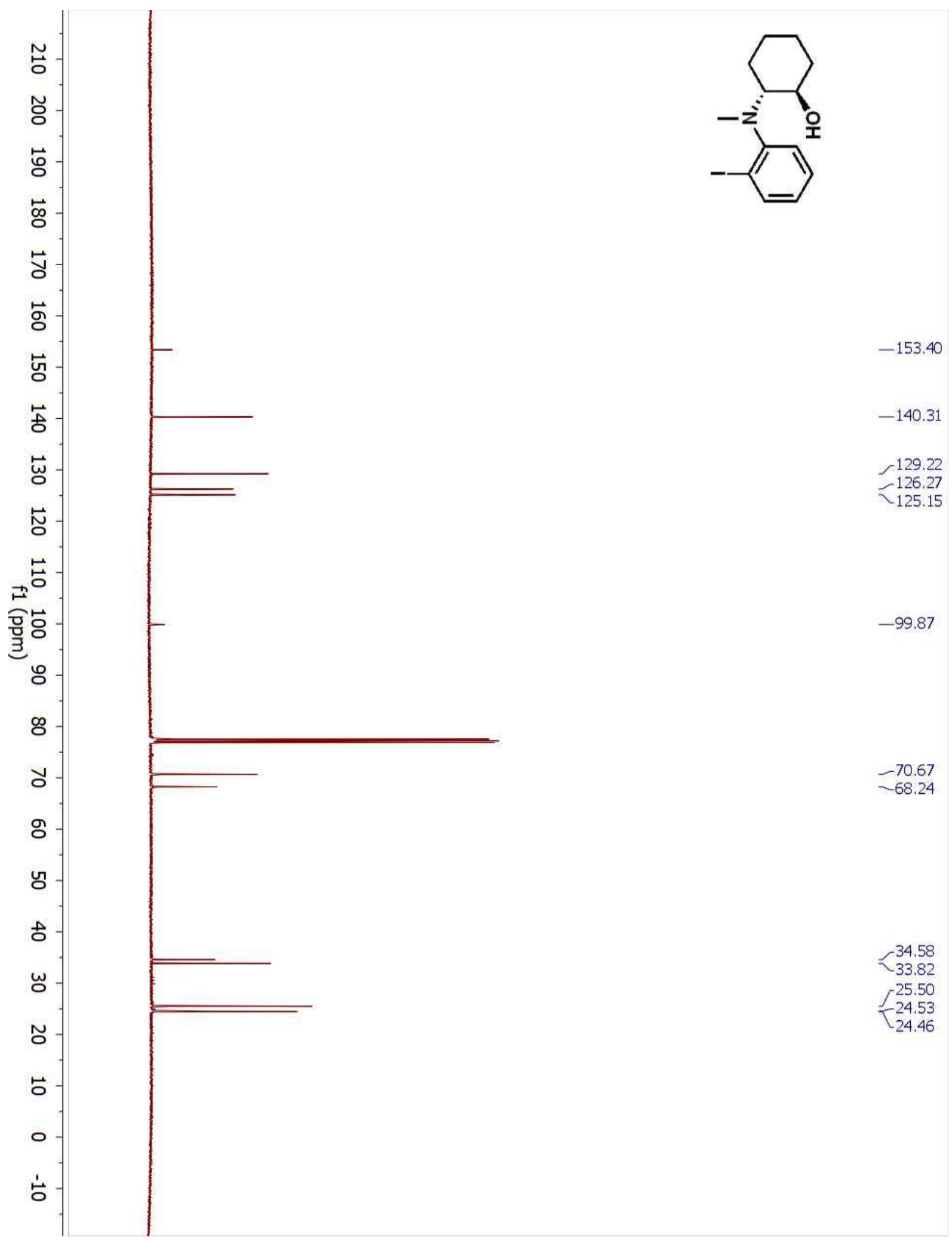

Figure S31. ${ }^{13} \mathrm{C}$ NMR spectrum of $1 e$ in $\mathrm{CDCl}_{3}$. 


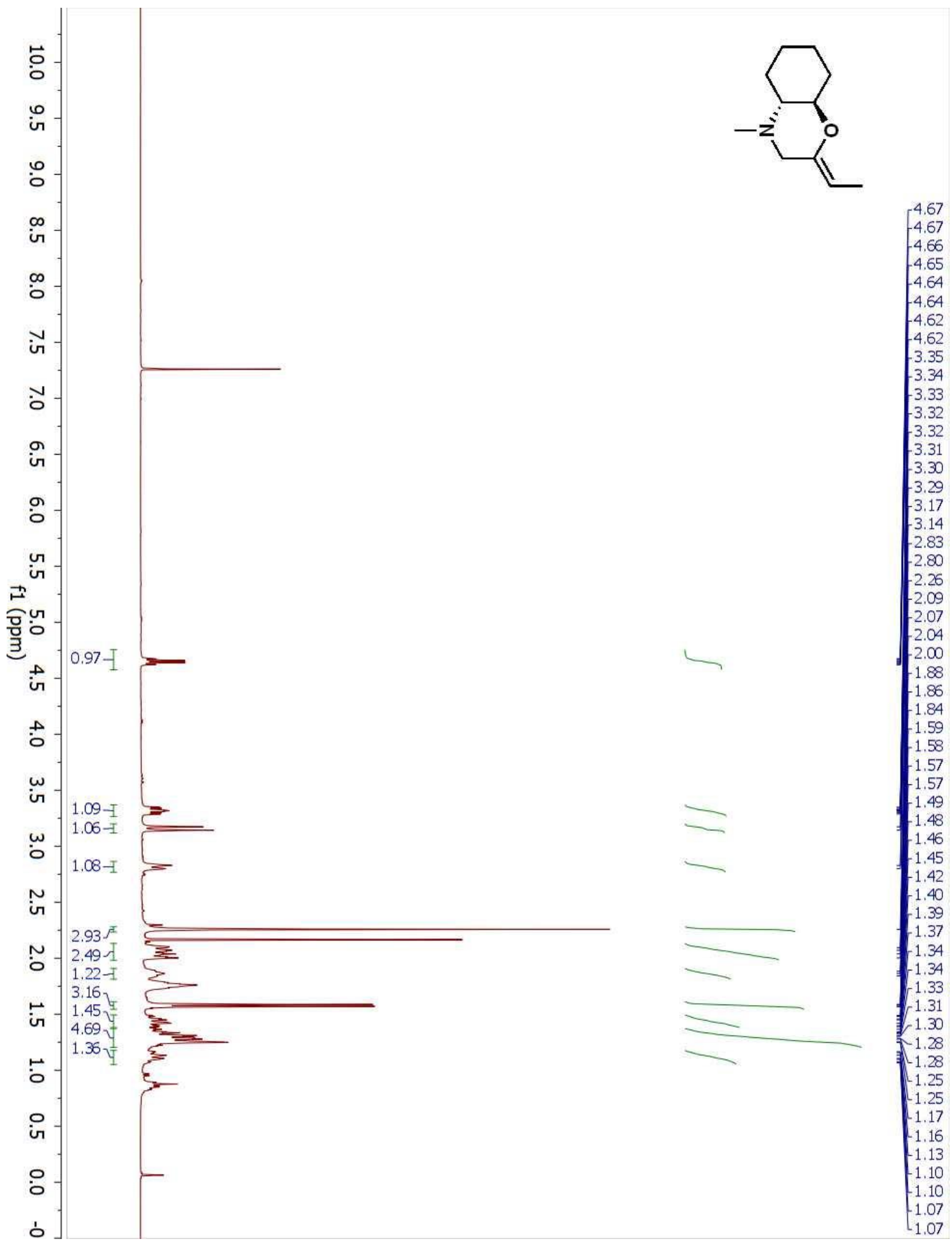

Figure S32. ${ }^{1} \mathrm{H} \mathrm{NMR}$ spectrum of $\mathbf{2 a}$ in $\mathrm{CDCl}_{3}$. 


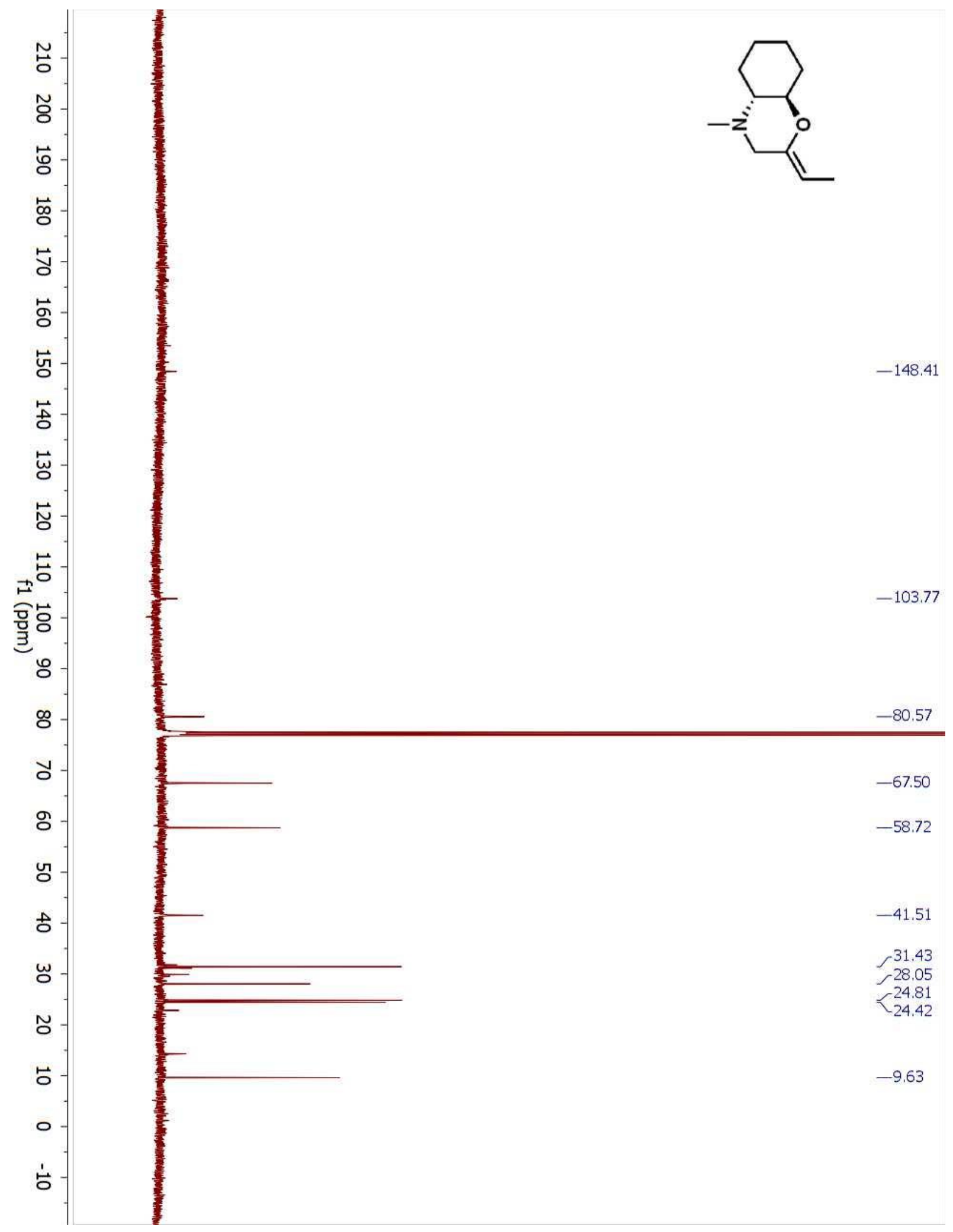

Figure S33. ${ }^{13} \mathrm{C}$ NMR spectrum of $\mathbf{2 a}$ in $\mathrm{CDCl}_{3}$. 


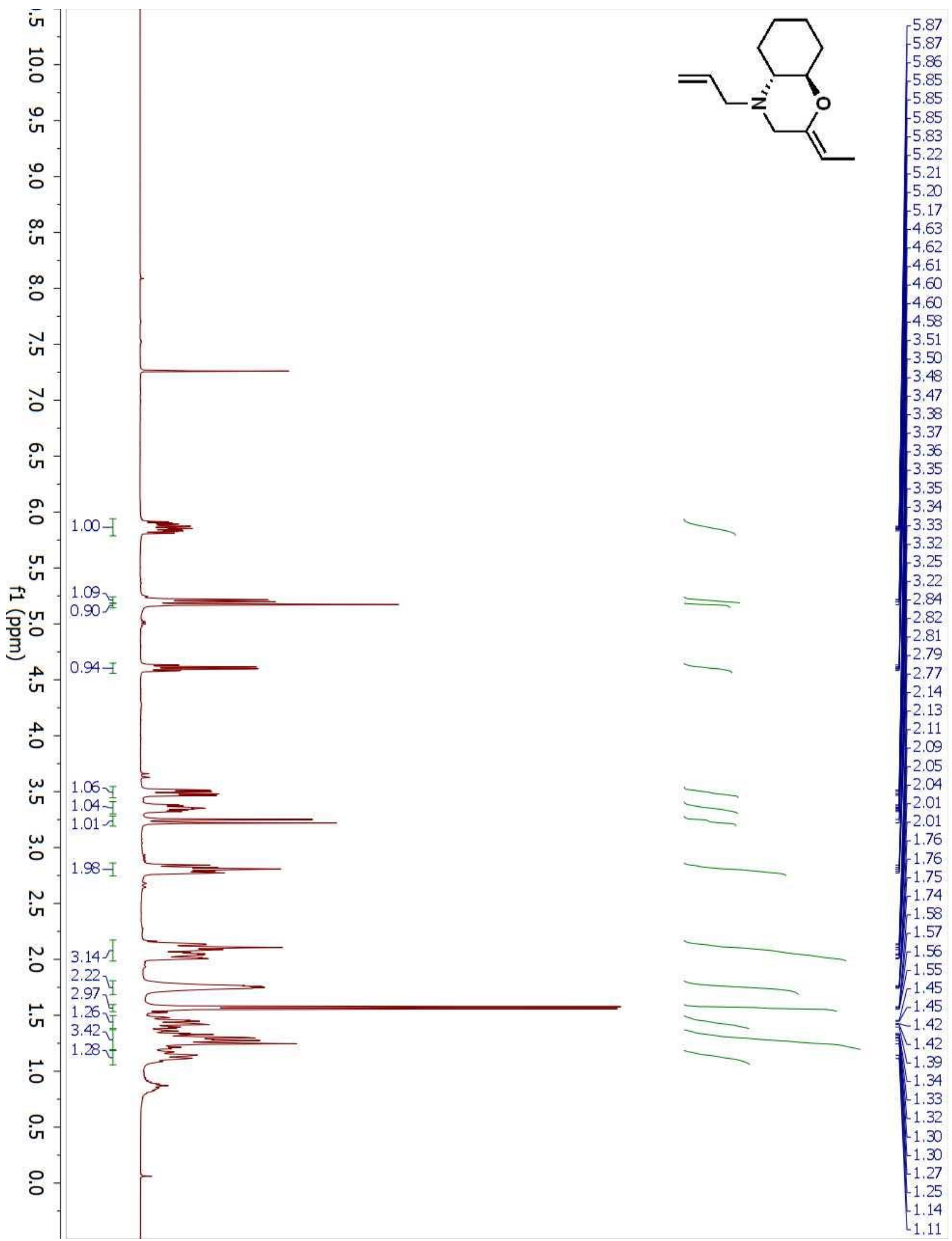

Figure S34. ${ }^{1} \mathrm{H}$ NMR spectrum of $\mathbf{2 d}$ in $\mathrm{CDCl}_{3}$. 


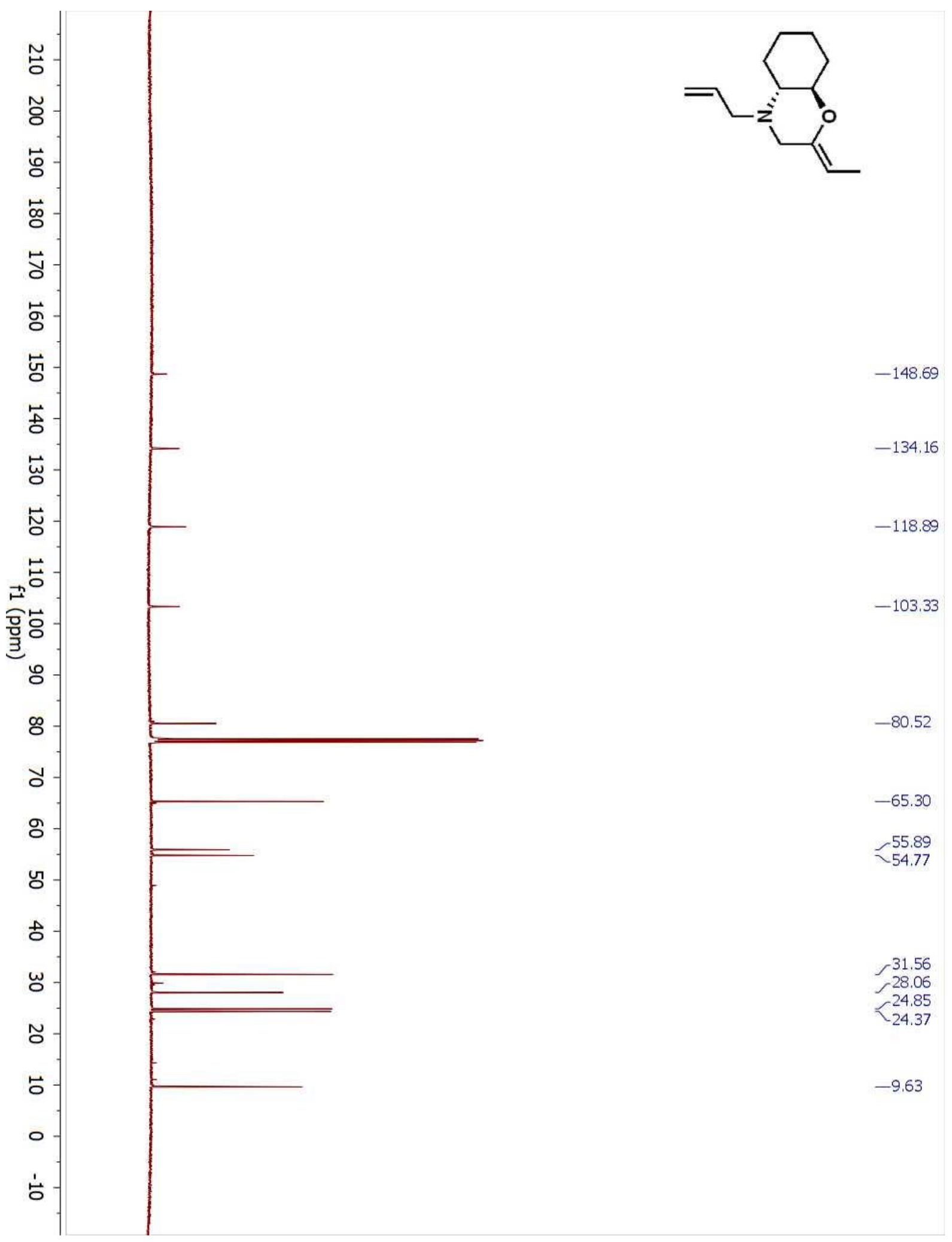

Figure S35. ${ }^{13} \mathrm{C}$ NMR spectrum of $\mathbf{2 d}$ in $\mathrm{CDCl}_{3}$. 


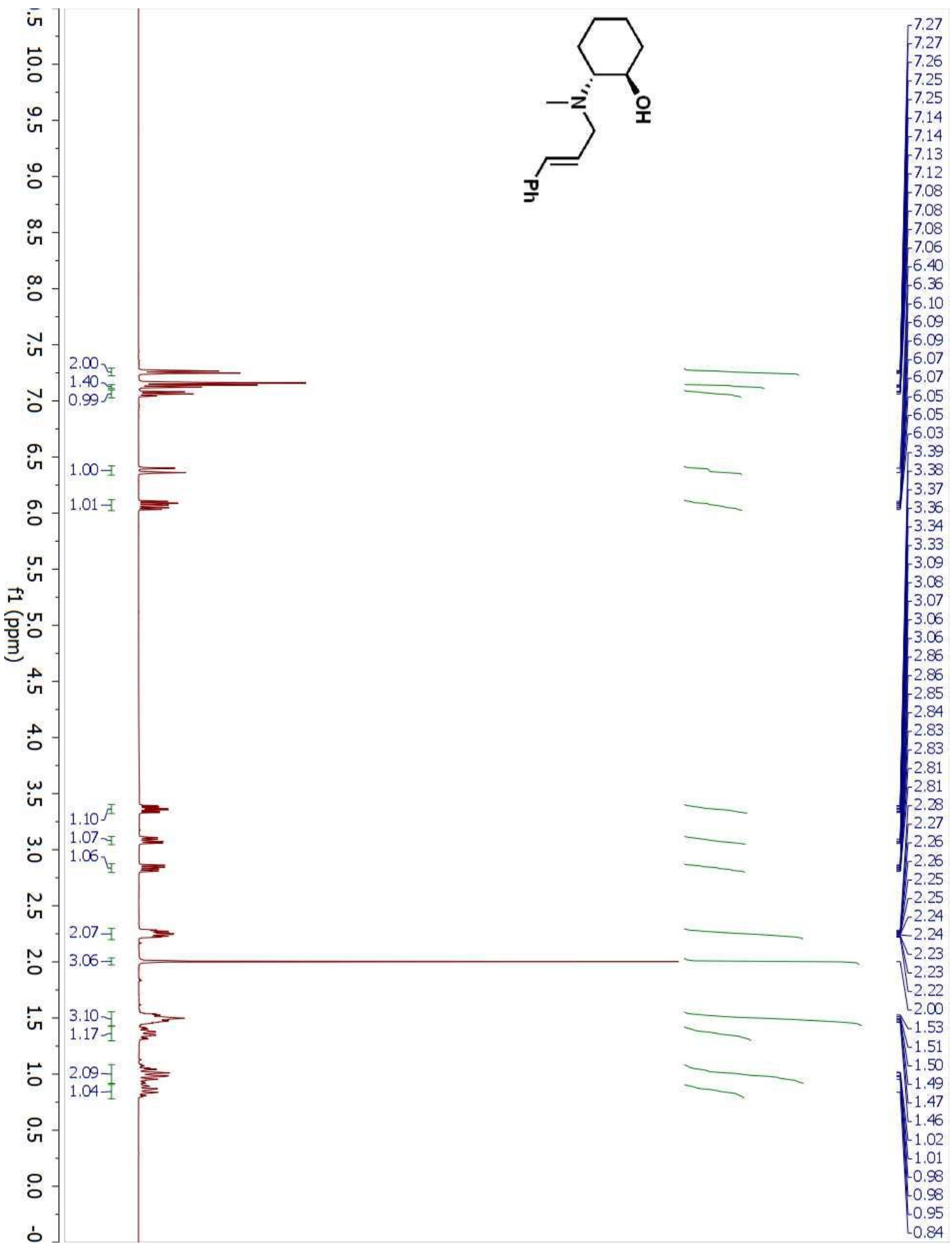

Figure S36. ${ }^{1} \mathrm{H}$ NMR spectrum of 3 in $\mathrm{C}_{6} \mathrm{D}_{6}$. 


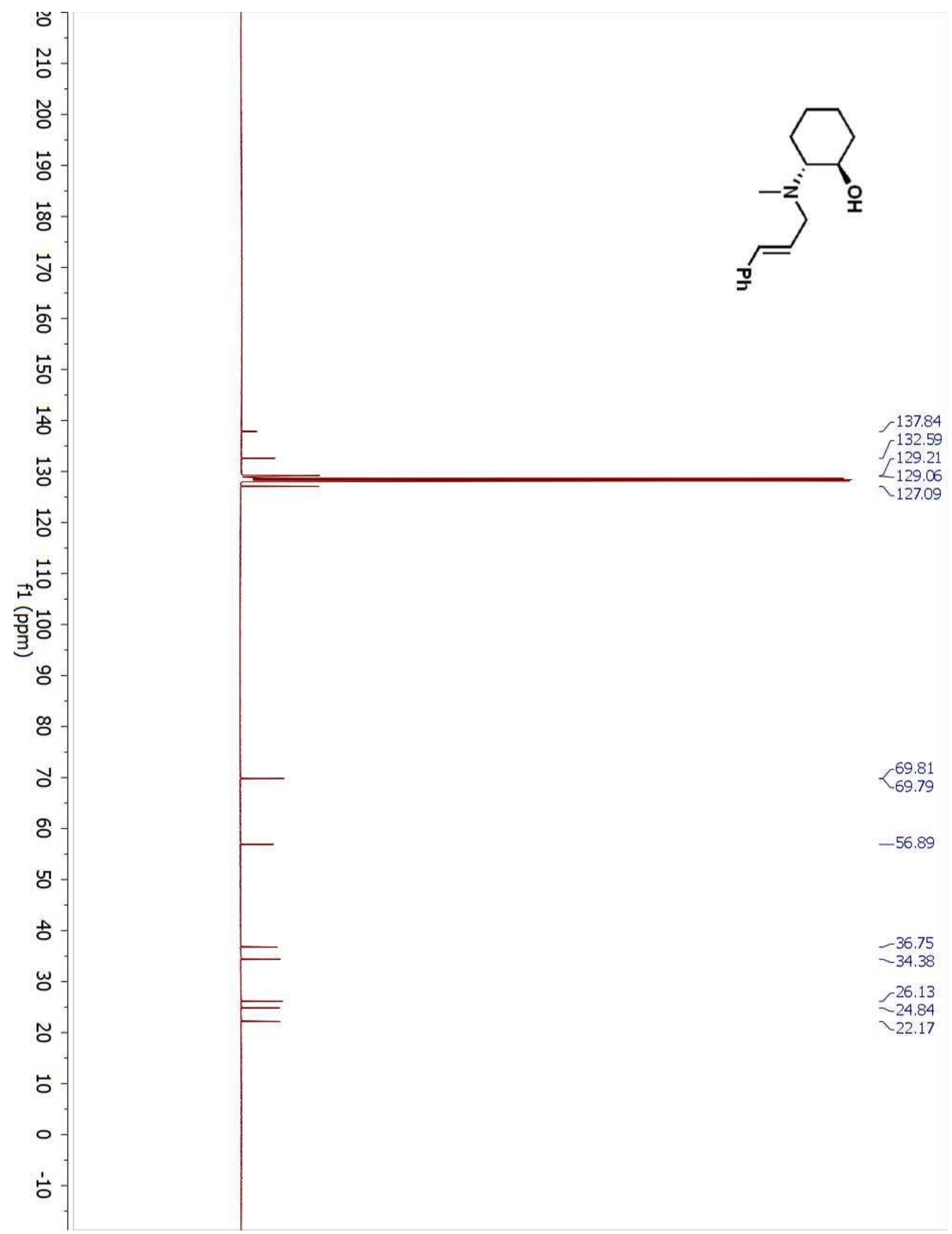

Figure S37. ${ }^{13} \mathrm{C}$ NMR spectrum of 3 in $\mathrm{C}_{6} \mathrm{D}_{6}$. 


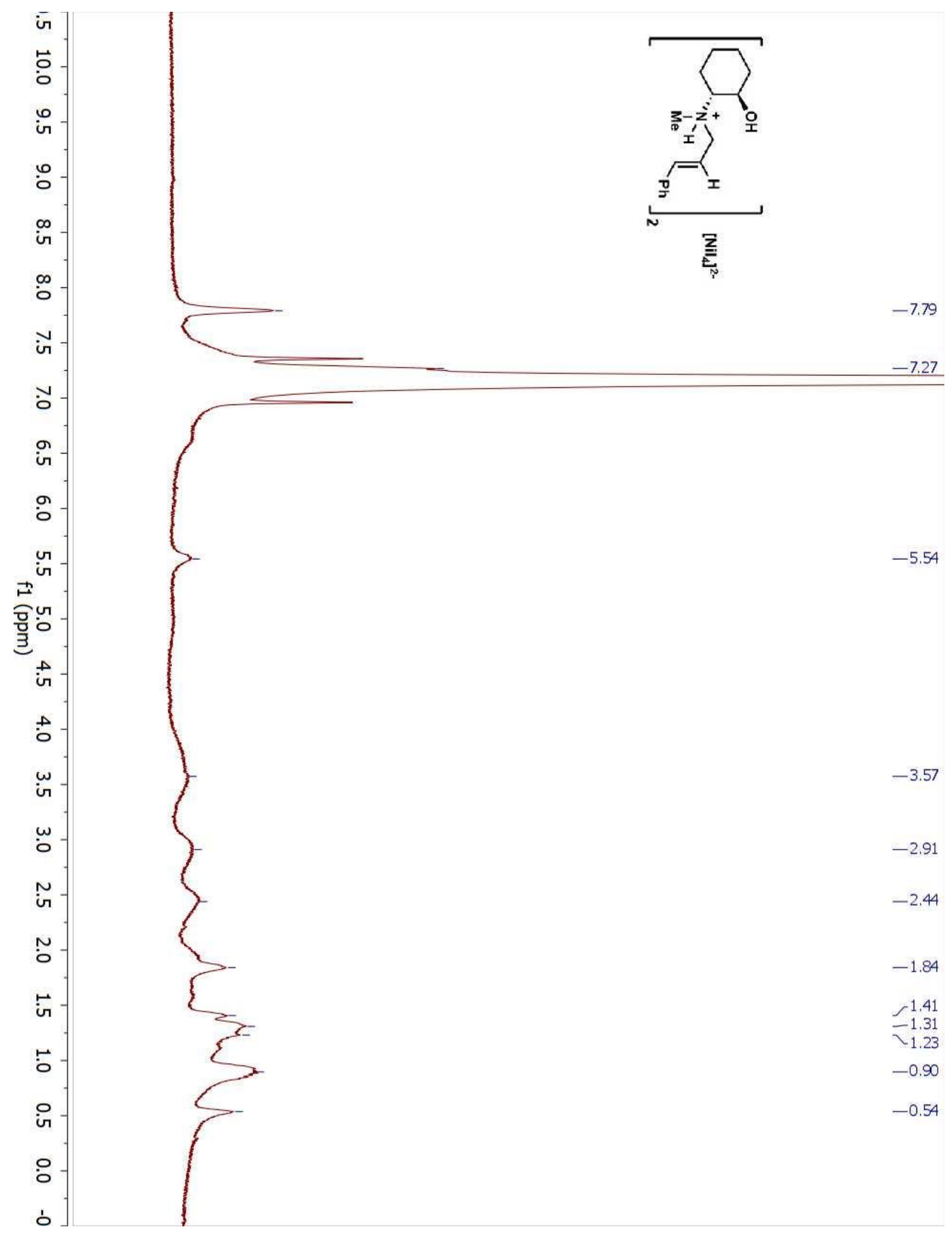

Figure S38. ${ }^{1} \mathrm{H}$ NMR spectrum of 4 in $\mathrm{C}_{6} \mathrm{D}_{6}$. 


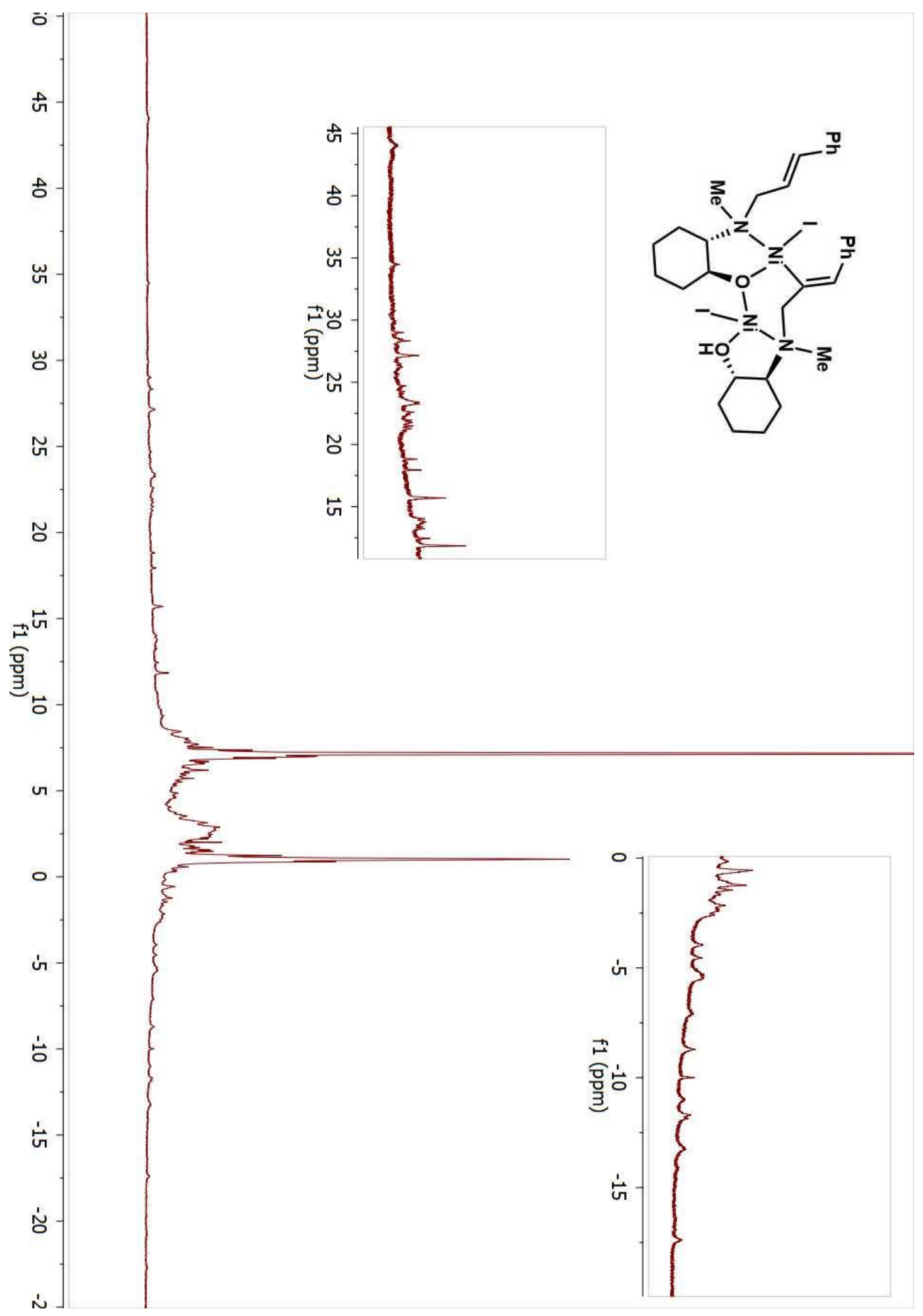

Figure S39. ${ }^{1} \mathrm{H}$ NMR spectrum of 5 in $\mathrm{C}_{6} \mathrm{D}_{6}$. 


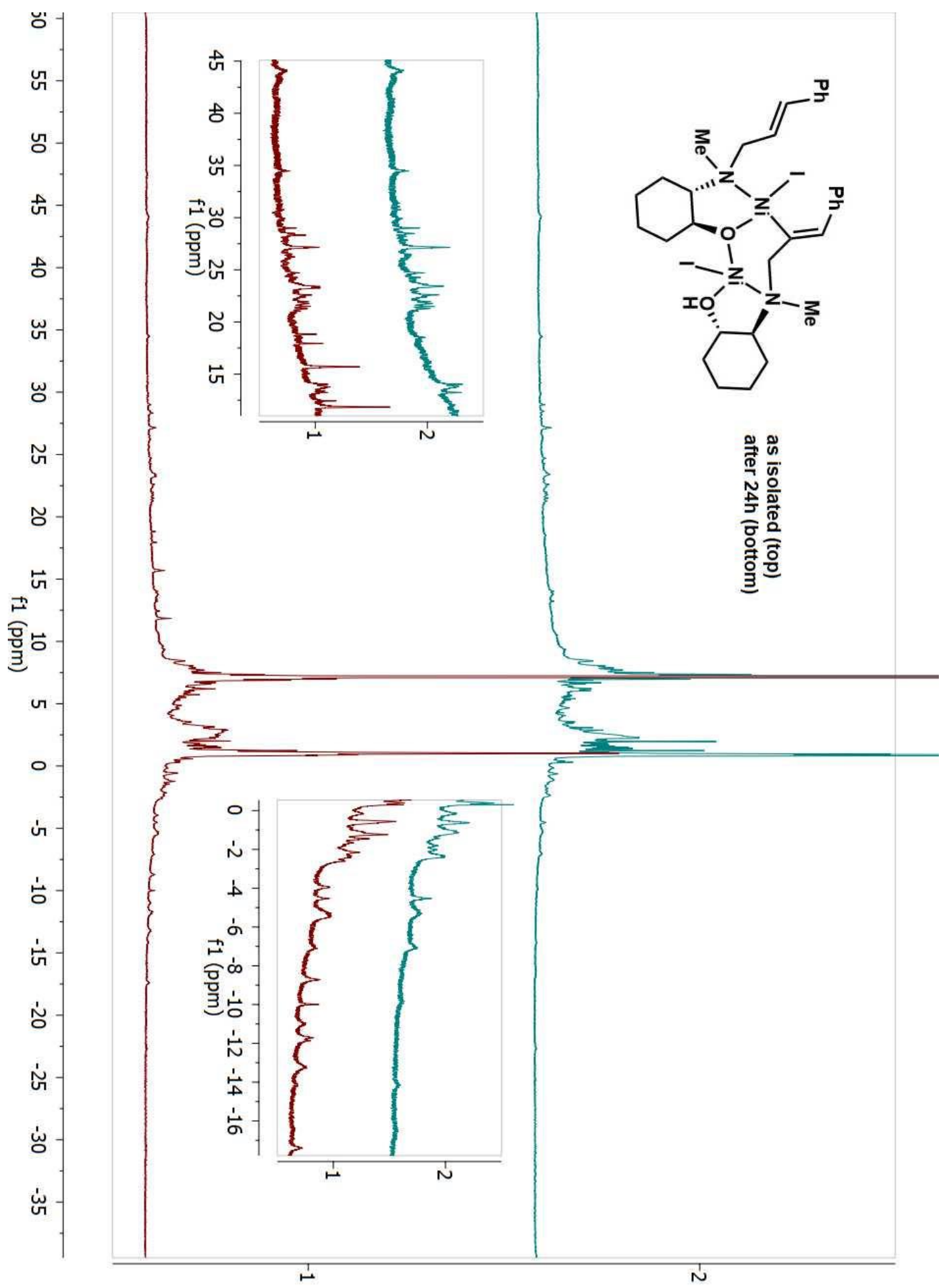

Figure S40. Stacked ${ }^{1} \mathrm{H}$ NMR spectra of compound 5 (top) and its decomposition products(s) $\mathbf{5}$, in $\mathrm{C}_{6} \mathrm{D}_{6}$. 


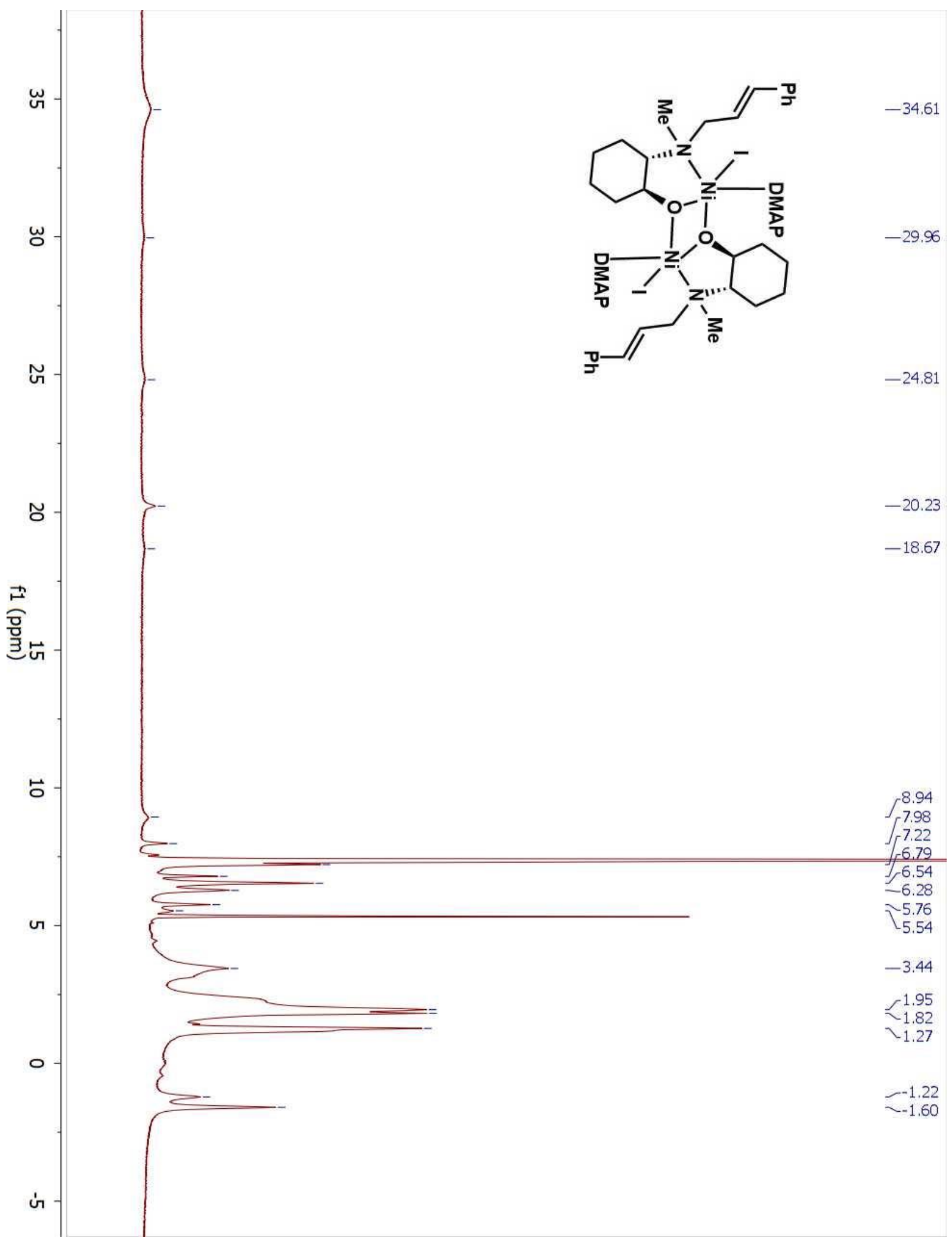

Figure S41. ${ }^{1} \mathrm{H}$ NMR spectrum of 6 in $\mathrm{CD}_{2} \mathrm{Cl}_{2}$. 


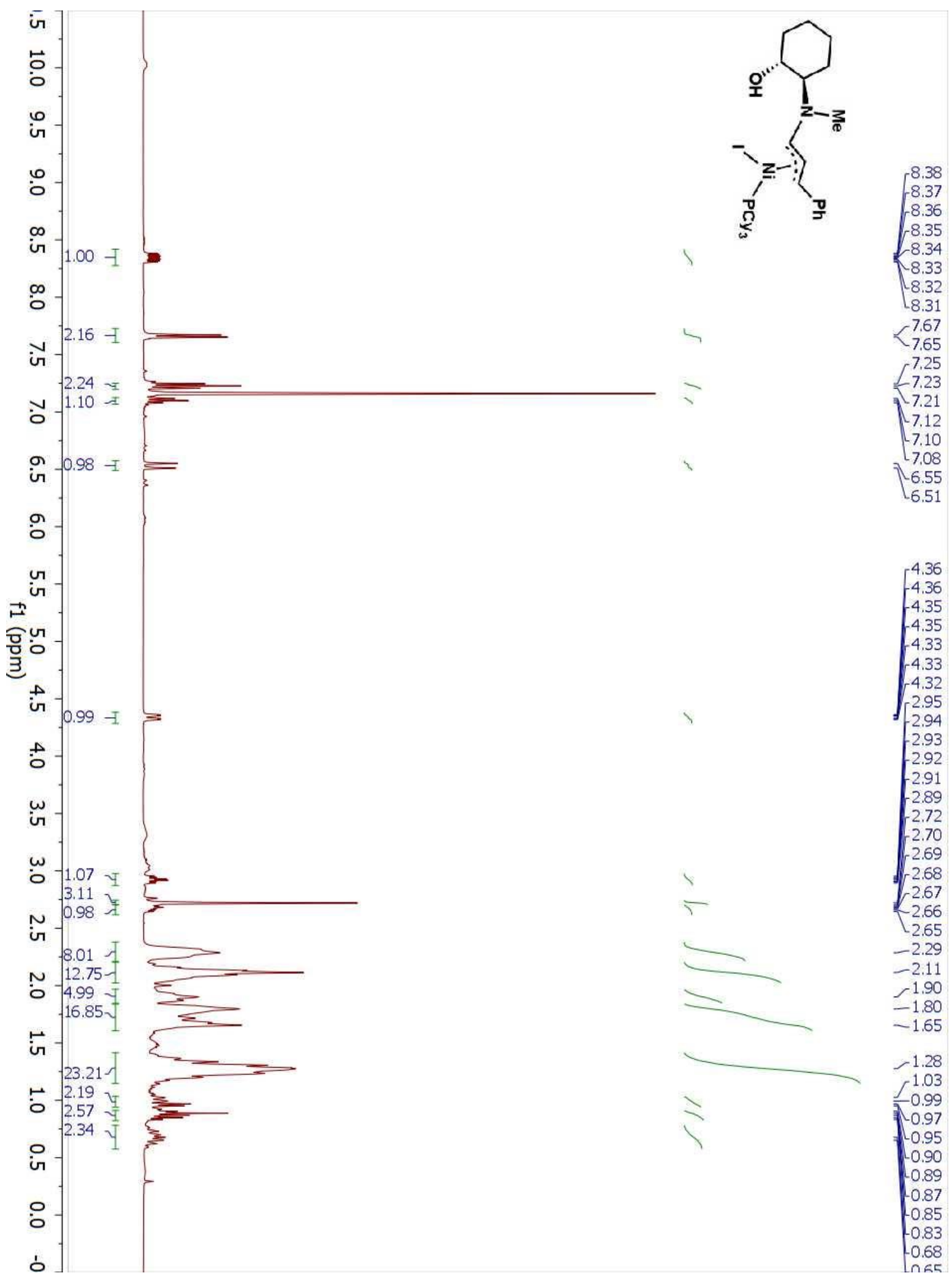

Figure S42. ${ }^{1} \mathrm{H}$ NMR spectrum of 7 in $\mathrm{C}_{6} \mathrm{D}_{6}$. 


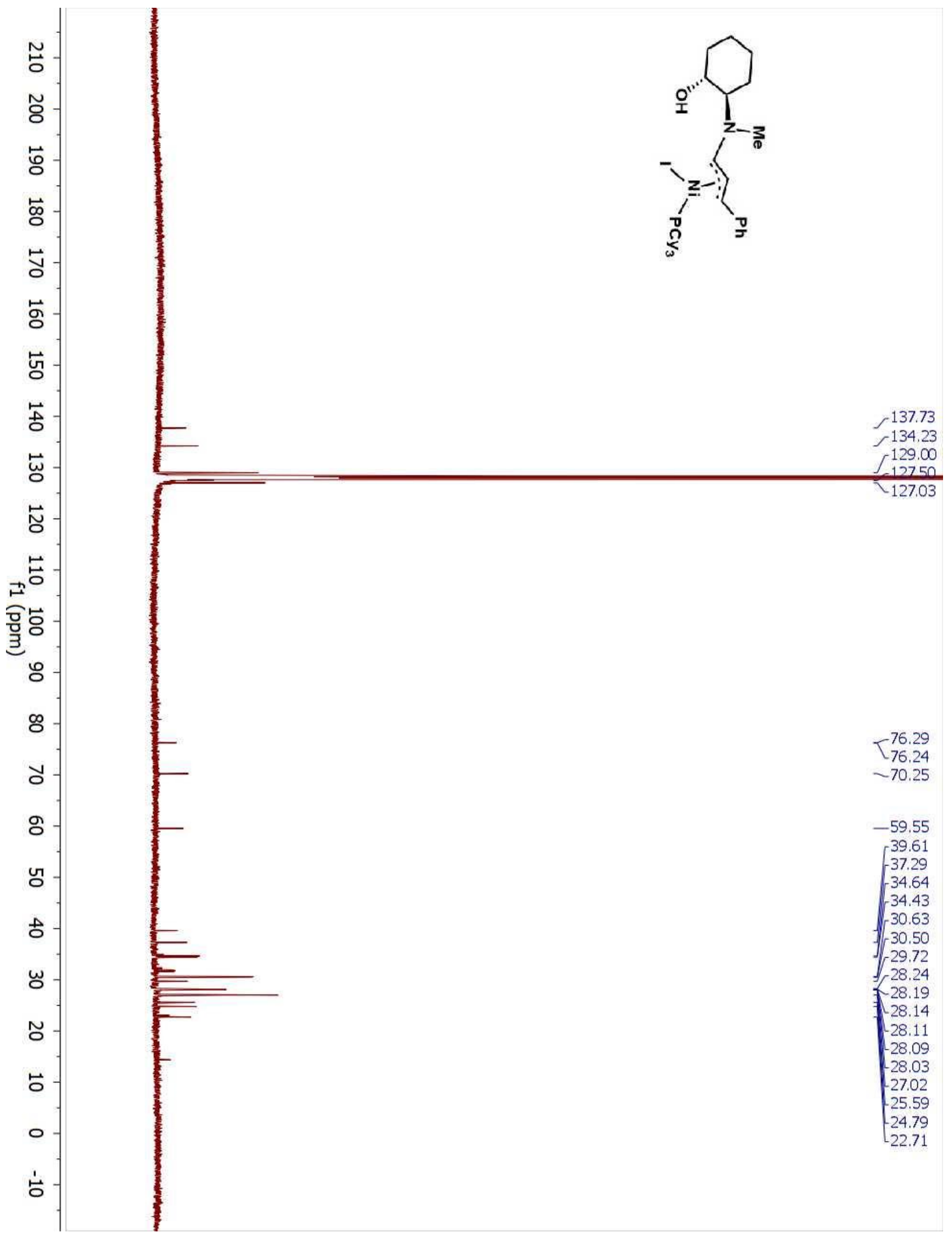

Figure S43. ${ }^{13} \mathrm{C}$ NMR spectrum of 7 in $\mathrm{C}_{6} \mathrm{D}_{6}$. 


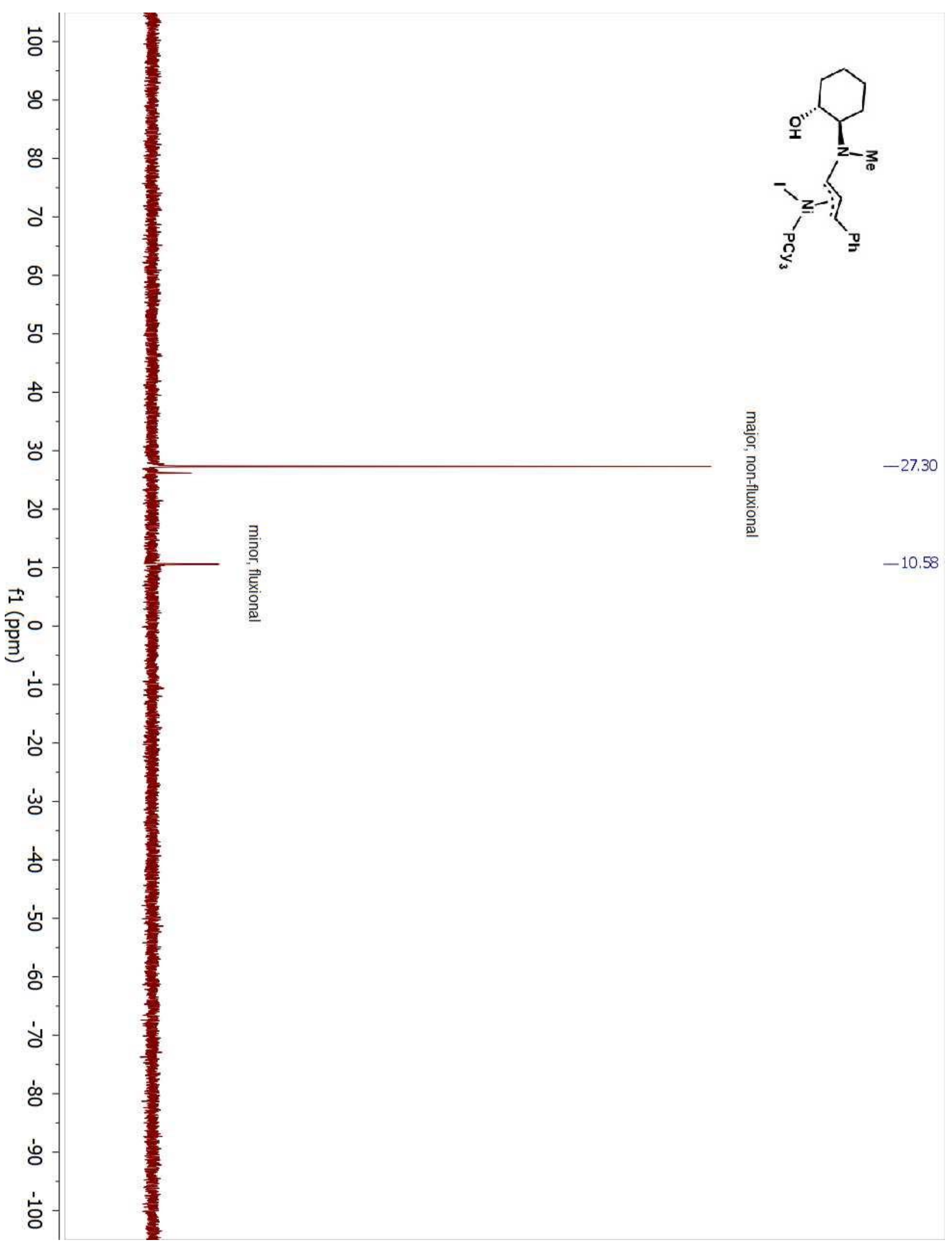

Figure S44. ${ }^{31} \mathrm{P}$ NMR spectrum of compound 7 in $\mathrm{C}_{6} \mathrm{D}_{6}$. The small, broadened upfield peak is associated with the minor diastereomer, which displayed broadened resonances in the ${ }^{1} \mathrm{H} N M R$ spectrum. 


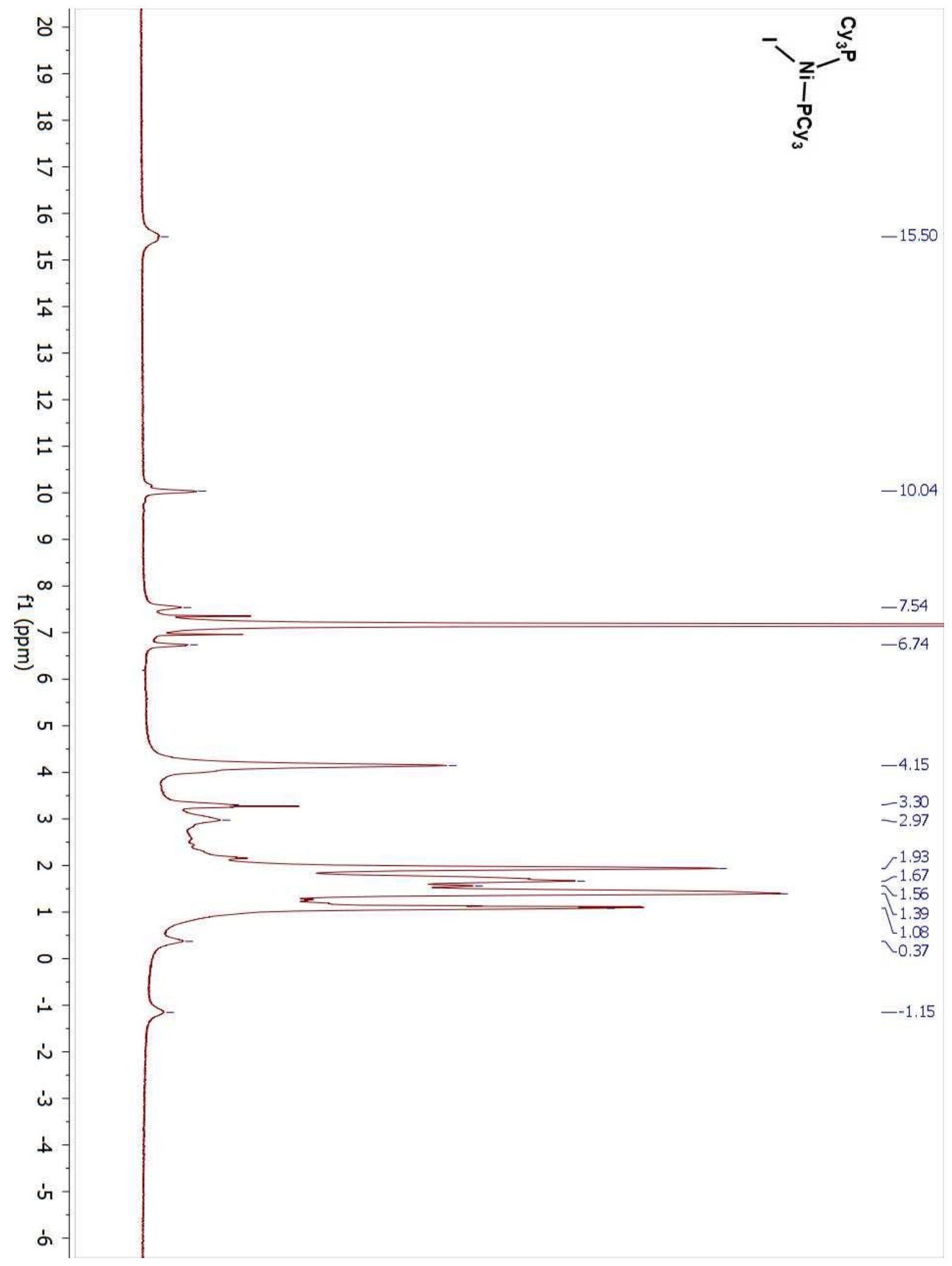

Figure S45. ${ }^{1} \mathrm{H}$ NMR spectrum of $\left(\mathrm{PCy}_{3}\right)_{2} \mathrm{NiI}$ in $\mathrm{C}_{6} \mathrm{D}_{6}$. 


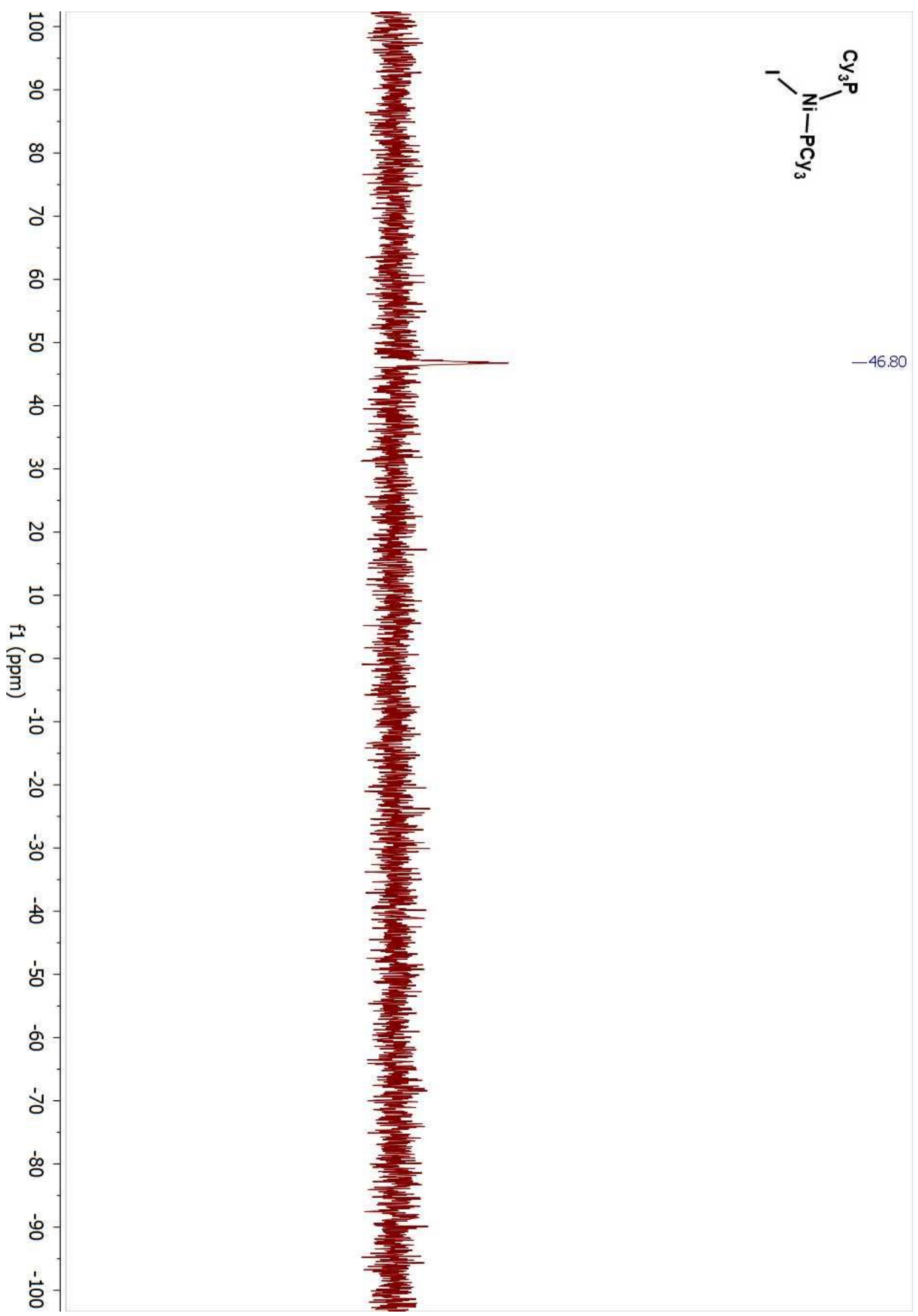

Figure 46. ${ }^{31} \mathrm{P}$ NMR spectrum of $\left(\mathrm{PCy}_{3}\right)_{2} \mathrm{NiI}$ in $\mathrm{C}_{6} \mathrm{D}_{6}$. 


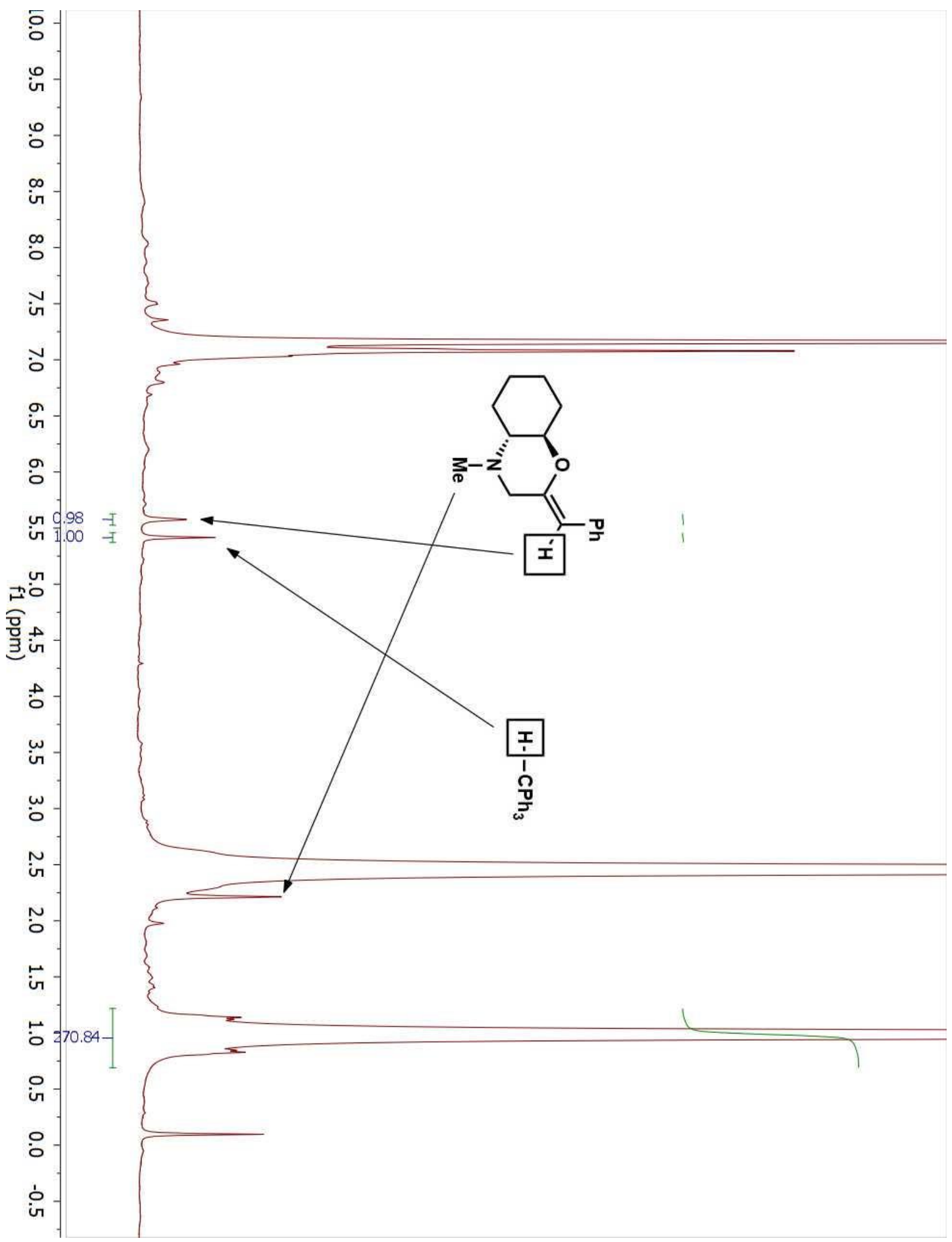

Figure S47. ${ }^{1} \mathrm{H}$ NMR spectrum of 5 following exposure to ca. 120 equiv of $\mathrm{Et}_{3} \mathrm{~N}$ in $\mathrm{C}_{6} \mathrm{D}_{6}$ for 30 minutes at $23{ }^{\circ} \mathrm{C}$. Key resonances assigned to $\mathbf{2 f}$ and a triphenylmethane internal standard are identified with arrows. Quantities: $8.0 \mathrm{mg}$ of 5, $1.0 \mathrm{mg}$ of $\mathrm{HCPh}_{3}$. Equivalents of $\mathrm{Et}_{3} \mathrm{~N}$ are estimated based on the relative integration of its resonances to that of $\mathrm{HCPh}_{3}$. 


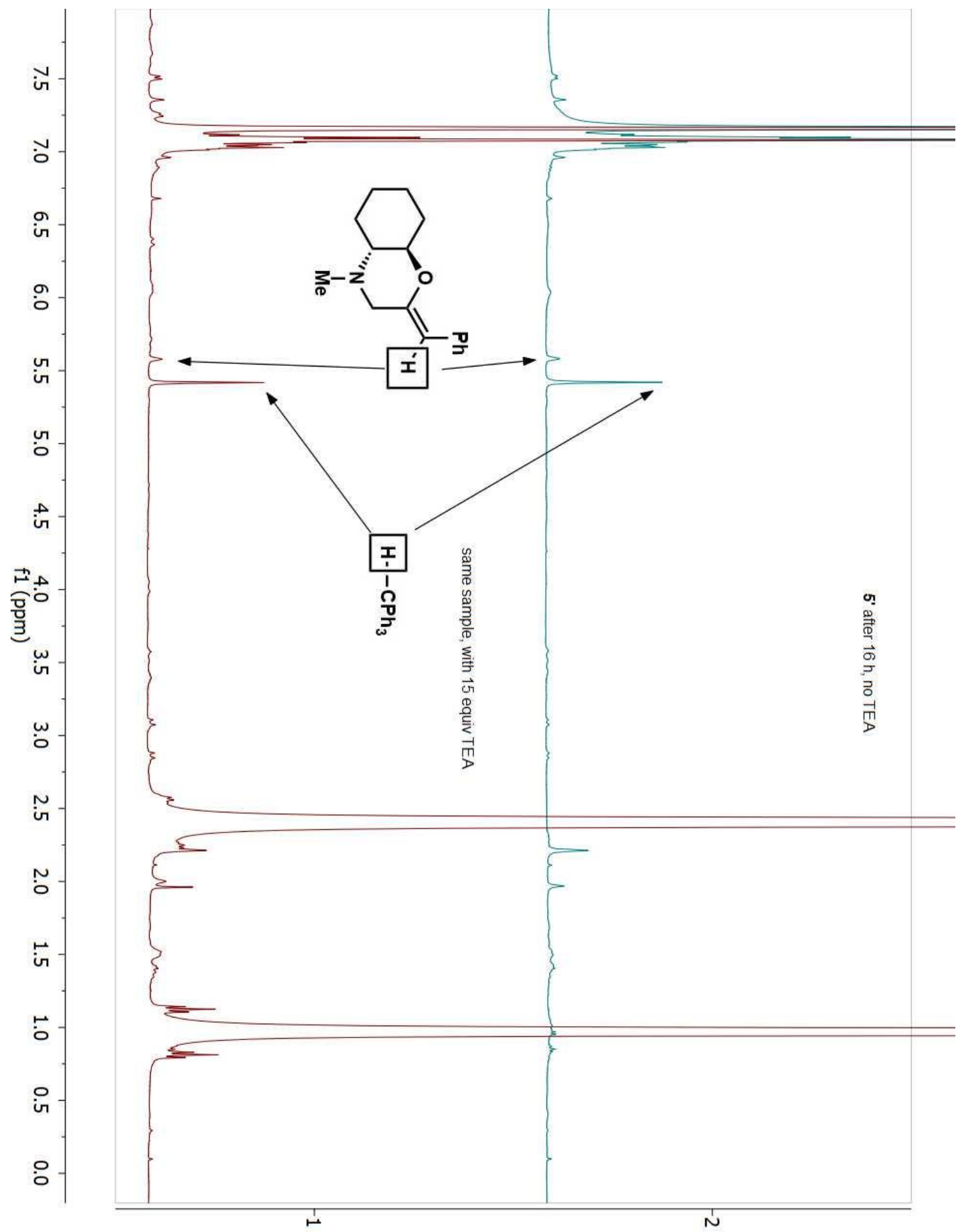

Figure S48. Stacked ${ }^{1} \mathrm{H}$ NMR spectra of $\mathbf{5}^{\prime}$, before and after the addition of ca. 15 equivalents of $\mathrm{Et}_{3} \mathrm{~N}$. $5.0 \mathrm{mg}$ of 5 were allowed to decompose at ca. $23{ }^{\circ} \mathrm{C}$ for $16 \mathrm{~h}$ with $1.1 \mathrm{mg}$ of a $\mathrm{HCPh}_{3}$ internal standard. The second spectrum was collected ca. $2 \mathrm{~h}$ following the addition of base. The ratio of $\mathbf{2 f}$ to $\mathrm{HCPh}_{3}$ is ca. 0.22:1.00 in both spectra, indicating a 19\% spectroscopic yield. 


\section{References}

1. Han, S.-J.; Doi, R.; Stoltz, B. M. Nickel-Catalyzed Intramolecular C-O Bond Formation: Synthesis of Cyclic Enol Ethers. Angew. Chem. Int. Ed. 2016, 55, 7437-7440.

2. Sekar, G.; Singh, V. K. An Efficient Method for Cleavage of Epoxides with Aromatic Amines. J. Org. Chem. 1999, 64, 287-289.

3. Hoberg, H.; Radine, K.; Krüger, C.; Romão, M. J. Synthesis of New Phosphine Nickel(I) Complexes and Crystal Structure of $\mu_{3}$-Iodo-tris-iodo-cyclotris(triphenylphosphine Nickel), (TPP) ${ }_{3} \mathrm{Ni}_{3} \mathrm{I}_{4}$. Z. Naturforsch. B 1985, 40b, 607-614.

4. APEX3, SADABS, and SAINT. Bruker AXS. Madison, WI, USA.

5. Sheldrick, G.M. SHELXT - Integrated space-group and crystal-structure determination. Acta Cryst. 2015, A71, 3-8.

6. Sheldrick, G.M. Crystal structure refinement with SHELXL. Acta Cryst. 2015, C71, 3-8.

7. Farrugia, L. J. WinGX and ORTEP for Windows: an update. J. Appl. Cryst. 2012, 45, 849-854.

8. Spek, A. L. PLATON SQUEEZE: a tool for the calculation of the disordered solvent contribution to the calculated structure factors. Acta Cryst. 2015, C71, 9-18.

9. Macrae, C. F.; Sovago, I.; Cottrell, S. J.; Galek, P. T. A.; McCabe, P.; Pidcock, E.; Platings, M.; Shields, G. P.; Stevens, J. S.; Towler, M.; Wood, P. A. Mercury 4.0: from visualization to analysis, design and prediction. J. Appl. Cryst. 2020, 53, 226-235.

10. (a) Neese, F. Software Update: The ORCA Program System, Version 4.0. Wiley Interdiscip. Rev.: Comput. Mol. Sci. 2018, 8, No. e1327. (b) Neese, F. The ORCA Program System. Wiley Interdiscip. Rev.: Comput. Mol. Sci. 2012, 2, 73-78.

11. Adamo, C.; Barone, V. Toward Reliable Density Functional Methods without Adjustable Parameters: The PBE0 Model. J. Chem. Phys. 1999, 110, 6158-6170.

12. (a) Caldeweyher, E.; Ehlert, S.; Hansen, A.; Neugebauer, H.; Spicher, S.; Bannwarth, C.; Grimme, S. A Generally Applicable Atomic-Charge Dependent London Dispersion Correction. J. Chem. Phys. 2019, 150 (15), 154122. (b) Caldeweyher, E.; Bannwarth, C.; Grimme, S. Extension of the D3 Dispersion Coefficient Model. J. Chem. Phys. 2017, 147 (3), 034112.

13. Weigend, F.; Ahlrichs, R. Balanced Basis Sets of Split Valence, Triple Zeta Valence and Quadruple Zeta Valence Quality for H to Rn: Design and Assessment of Accuracy. Phys. Chem. Chem. Phys. 2005, 7 (18), 3297-3305.

14. Peterson, K. A.; Figgen, D.; Goll, E.; Stoll, H.; Dolg, M. Systematically Convergent Basis Sets with Relativistic Pseudopotentials. II. Small-Core Pseudopotentials and Correlation Consistent Basis Sets for the Post- $d$ Group 16-18 Elements. The Journal of Chemical Physics 2003, 119 (21), 11113-11123.

15. Barone, V.; Cossi, M. Quantum Calculation of Molecular Energies and Energy Gradients in Solution by a Conductor Solvent Model. J. Phys. Chem. A 1998, 102 (11), 1995-2001.

16. Garcia-Ratés, M.; Neese, F. Effect of the Solute Cavity on the Solvation Energy and Its Derivatives within the Framework of the Gaussian Charge Scheme. Journal of Computational Chemistry 2020, 41 (9), 922-939.

17. Ishida, K.; Morokuma, K.; Komornicki, A. The Intrinsic Reaction Coordinate. An Ab Initio Calculation for $\mathrm{HNC} \rightarrow \mathrm{HCN}$ and $\mathrm{H}-+\mathrm{CH} 4 \rightarrow \mathrm{CH} 4+\mathrm{H}^{-}$. J. Chem. Phys. 1977, 66 (5), 2153-2156.

18. Zhao, Y.; Truhlar, D. G. The M06 Suite of Density Functionals for Main Group Thermochemistry, Thermochemical Kinetics, Noncovalent Interactions, Excited States, and Transition Elements: Two New Functionals and Systematic Testing of Four M06-Class Functionals and 12 Other Functionals. Theor Chem Account 2008, 120 (1), 215-241.

19. Grimme, S. Supramolecular Binding Thermodynamics by Dispersion-Corrected Density Functional Theory. Chemistry - A European Journal 2012, 18 (32), 9955-9964.

20. Izato, Y.; Matsugi, A.; Koshi, M.; Miyake, A. A Simple Heuristic Approach to Estimate the Thermochemistry of Condensed-Phase Molecules Based on the Polarizable Continuum Model. Phys. Chem. Chem. Phys. 2019, 21 (35), 18920-18929.

21. Finkelstein, A. V.; Janin, J. The Price of Lost Freedom: Entropy of Bimolecular Complex Formation. Protein Engineering, Design and Selection 1989, 3 (1), 1-3.

22. Neese, F.; Wennmohs, F.; Hansen, A.; Becker, U. Efficient, Approximate and Parallel Hartree-Fock and Hybrid DFT Calculations. A 'Chain-of-Spheres' Algorithm for the Hartree-Fock Exchange. Chemical Physics 2009, $356,98-109$.

23. Weigend, F. Accurate Coulomb-Fitting Basis Sets for H to Rn. Phys. Chem. Chem. Phys. 2006, 8 (9), 10571065 . 
24. Stoychev, G. L.; Auer, A. A.; Neese, F. Automatic Generation of Auxiliary Basis Sets. J. Chem. Theory Comput. 2017, 13, 554-562.

25. (a) Ferré, N.; Guihéry, N.; Malrieu, J.-P. Spin Decontamination of Broken-Symmetry Density Functional Theory Calculations: Deeper Insight and New Formulations. Phys. Chem. Chem. Phys. 2015, 17 (22), 1437514382. (b) Neese, F. Definition of Corresponding Orbitals and the Diradical Character in Broken Symmetry DFT Calculations on Spin Coupled Systems. Journal of Physics and Chemistry of Solids 2004, 65 (4), 781-785.

26. Yamaguchi, K.; Takahara, Y.; Fueno, T. Ab-Initio Molecular Orbital Studies of Structure and Reactivity of Transition Metal-OXO Compounds. In Applied Quantum Chemistry; Smith, V. H., Schaefer, H. F., Morokuma, K., Eds.; Springer Netherlands: Dordrecht, 1986; pp 155-184.

27. (a) Schleyer, P. von R.; Maerker, C.; Dransfeld, A.; Jiao, H.; van Eikema Hommes, N. J. R. NucleusIndependent Chemical Shifts: A Simple and Efficient Aromaticity Probe. J. Am. Chem. Soc. 1996, 118 (26), 6317-6318. (b) Chen, Z.; Wannere, C. S.; Corminboeuf, C.; Puchta, R.; Schleyer, P. von R. NucleusIndependent Chemical Shifts (NICS) as an Aromaticity Criterion. Chem. Rev. 2005, 105 (10), 3842-3888.

28. (a) Riplinger, C.; Neese, F. An Efficient and near Linear Scaling Pair Natural Orbital Based Local Coupled Cluster Method. J. Chem. Phys. 2013, 138 (3), 034106. (b) Riplinger, C.; Sandhoefer, B.; Hansen, A.; Neese, F. Natural Triple Excitations in Local Coupled Cluster Calculations with Pair Natural Orbitals. J. Chem. Phys. 2013, 139 (13), 134101. (c) Riplinger, C.; Pinski, P.; Becker, U.; Valeev, E. F.; Neese, F. Sparse Maps-A Systematic Infrastructure for Reduced-Scaling Electronic Structure Methods. II. Linear Scaling Domain Based Pair Natural Orbital Coupled Cluster Theory. J. Chem. Phys. 2016, 144 (2), 024109.

29. Bartlett, R. J.; Musiał, M. Coupled-Cluster Theory in Quantum Chemistry. Rev. Mod. Phys. 2007, 79 (1), 291352 .

30. (a) Bertels, L. W.; Lee, J.; Head-Gordon, M. Polishing the Gold Standard: The Role of Orbital Choice in CCSD(T) Vibrational Frequency Prediction. J. Chem. Theory Comput. 2021, 17 (2), 742-755. (b) Fang, Z.; Lee, Z.; Peterson, K. A.; Dixon, D. A. Use of Improved Orbitals for CCSD(T) Calculations for Predicting Heats of Formation of Group IV and Group VI Metal Oxide Monomers and Dimers and UCl6. J. Chem. Theory Comput. 2016, 12 (8), 3583-3592. (c) Fang, Z.; Vasiliu, M.; Peterson, K. A.; Dixon, D. A. Prediction of Bond Dissociation Energies/Heats of Formation for Diatomic Transition Metal Compounds: CCSD(T) Works. J. Chem. Theory Comput. 2017, 13 (3), 1057-1066.

31. Minenkov, Y.; Wang, H.; Wang, Z.; Sarathy, S. M.; Cavallo, L. Heats of Formation of Medium-Sized Organic Compounds from Contemporary Electronic Structure Methods. J. Chem. Theory Comput. 2017, 13 (8), 35373560 .

32. $\mathrm{T}_{1}$ diagnostic defined as the norm of the singles amplitude vector divided by the square root of the number of electrons

33. Stephens, P. J.; Devlin, F. J.; Chabalowski, C. F.; Frisch, M. J. Ab Initio Calculation of Vibrational Absorption and Circular Dichroism Spectra Using Density Functional Force Fields. J. Phys. Chem. 1994, 98 (45), 1162311627.

34. Mardirossian, N.; Head-Gordon, M. $\omega B 97 M-V$ : A Combinatorially Optimized, Range-Separated Hybrid, MetaGGA Density Functional with VV10 Nonlocal Correlation. J. Chem. Phys. 2016, 144 (21), 214110.

35. Yu, H. S.; He, X.; Li, S. L.; Truhlar, D. G. MN15: A Kohn-Sham Global-Hybrid Exchange-Correlation Density Functional with Broad Accuracy for Multi-Reference and Single-Reference Systems and Noncovalent Interactions. Chem. Sci. 2016, 7 (8), 5032-5051.

36. For additional examples and discussion see: Funes-Ardoiz, I.; Nelson, D. J.; Maseras, F. Halide Abstraction Competes with Oxidative Addition in the Reactions of Aryl Halides with $\left[\mathrm{Ni}\left(\mathrm{PMe}_{n} \mathrm{Ph}_{(3-n)}\right)_{4}\right]$. Chem. Eur. J. 2017, 23 (66), 16728-16733.

37. Nelsen, S. F.; Blackstock, S. C.; Kim, Y. Estimation of Inner Shell Marcus Terms for Amino Nitrogen Compounds by Molecular Orbital Calculations. J. Am. Chem. Soc. 1987, 109 (3), 677-682.

38. For examples of facile comproportionation from $\mathrm{Ni}(\mathrm{III})+\mathrm{Ni}$ (I) to $2 \mathrm{Ni}$ (II), see (a) Sun, R.; Qin, Y.; Ruccolo, S.; Schnedermann, C.; Costentin, C.; Nocera, D. G. Elucidation of a Redox-Mediated Reaction Cycle for NickelCatalyzed Cross Coupling. J. Am. Chem. Soc. 2019, 141 (1), 89-93. (b) Kawamata, Y.; Vantourout, J. C.; Hickey, D. P.; Bai, P.; Chen, L.; Hou, Q.; Qiao, W.; Barman, K.; Edwards, M. A.; Garrido-Castro, A. F.; deGruyter, J. N.; Nakamura, H.; Knouse, K.; Qin, C.; Clay, K. J.; Bao, D.; Li, C.; Starr, J. T.; Garcia-Irizarry, C.; Sach, N.; White, H. S.; Neurock, M.; Minteer, S. D.; Baran, P. S. Electrochemically Driven, Ni-Catalyzed Aryl Amination: Scope, Mechanism, and Applications. J. Am. Chem. Soc. 2019, 141 (15), 6392-6402.

39. Knizia, G. Intrinsic Atomic Orbitals: An Unbiased Bridge between Quantum Theory and Chemical Concepts. $J$. Chem. Theory Comput. 2013, 9 (11), 4834-4843. 